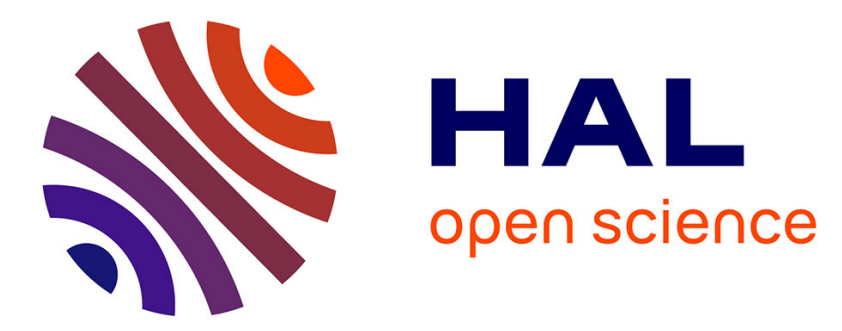

\title{
The Vector Electric Field Investigation (VEFI) on the C/NOFS Satellite
}

Robert F. Pfaff, P. Uribe, R. Fourre, J. Kujawski, N. Maynard, M. Acuña, D Rowland, H Freudenreich, K Bromund, S Martin, et al.

\section{- To cite this version:}

Robert F. Pfaff, P. Uribe, R. Fourre, J. Kujawski, N. Maynard, et al.. The Vector Electric Field Investigation (VEFI) on the C/NOFS Satellite. Space Science Reviews, 2021, 217, pp.85. 10.1007/s11214021-00859-y . insu-03502515

\section{HAL Id: insu-03502515 https://hal-insu.archives-ouvertes.fr/insu-03502515}

Submitted on 25 Dec 2021

HAL is a multi-disciplinary open access archive for the deposit and dissemination of scientific research documents, whether they are published or not. The documents may come from teaching and research institutions in France or abroad, or from public or private research centers.
L'archive ouverte pluridisciplinaire HAL, est destinée au dépôt et à la diffusion de documents scientifiques de niveau recherche, publiés ou non, émanant des établissements d'enseignement et de recherche français ou étrangers, des laboratoires publics ou privés. 


\title{
The Vector Electric Field Investigation (VEFI) on the C/NOFS Satellite
}

\author{
R. Pfaff ${ }^{1}$ (D) P. Uribe ${ }^{1} \cdot$ R. Fourre ${ }^{1} \cdot$ J. Kujawski ${ }^{1} \cdot$ N. Maynard ${ }^{2} \cdot$ M. Acuña ${ }^{1} \cdot$ D. Rowland ${ }^{1}$. \\ H. Freudenreich ${ }^{1} \cdot$ K. Bromund ${ }^{1}$. S. Martin ${ }^{1}$. C. Liebrecht ${ }^{1}$ - R. Kramer ${ }^{3}$. F. Hunsaker ${ }^{1}$. \\ R. Holzworth ${ }^{4} \cdot$ M. McCarthy ${ }^{4}$ - W. Farrell ${ }^{1}$ - J. Klenzing ${ }^{1} \cdot$ G. Le ${ }^{1} \cdot$ A. Jacobson ${ }^{4}$. \\ J. Houser ${ }^{1} \cdot$ C. Steigies ${ }^{1} \cdot$ J.-J. Berthelier ${ }^{5}$
}

Received: 13 July 2021 / Accepted: 9 October 2021 / Published online: 22 November 2021 This is a U.S. government work and not under copyright protection in the U.S.; foreign copyright protection may apply 2021

Abstract

The Vector Electric Field Investigation (VEFI) on the C/NOFS satellite comprises a suite of sensors controlled by one central electronics box. The primary measurement consists of a vector DC and AC electric field detector which extends spherical sensors with embedded pre-amps at the ends of six, 9.5-m booms forming three orthogonal detectors with baselines of $20 \mathrm{~m}$ tip-to-tip each. The primary VEFI measurement is the DC electric field at 16 vectors/sec with an accuracy of $0.5 \mathrm{mV} / \mathrm{m}$. The electric field receiver also measures the broad spectra of irregularities associated with equatorial spread-F and related ionospheric processes that create the scintillations responsible for the communication and navigation outages for which the C/NOFS mission is designed to understand and predict. The AC electric field measurements range from ELF to HF frequencies.

VEFI includes a flux-gate magnetometer providing DC measurements at 1 vector/sec and AC-coupled measurements at 16 vector/sec, as well as a fast, fixed-bias Langmuir probe that serves as the input signal to trigger the VEFI burst memory collection of high time resolution wave data when plasma density depletions are encountered in the low latitude nighttime ionosphere. A bi-directional optical lightning detector designed by the University of Washington (UW) provides continuous average lightning counts at different irradiance levels as well as high time resolution optical lightning emissions captured in the burst memory. The VEFI central electronics box receives inputs from all of the sensors and includes a configurable burst memory with 1-8 channels at sample rates as high as $32 \mathrm{ks} / \mathrm{s}$ per channel. The VEFI instrument is thus one experiment with many sensors. All of the instruments were designed, built, and tested at the NASA/Goddard Space Flight Center with the exception of the lightning detector which was designed at UW. The entire VEFI instrument was delivered on budget in less than 2 years.

VEFI included a number of technical advances and innovative features described in this article. These include: (1) Two independent sets of 3-axis, orthogonal electric field double probes; (2) Motor-driven, pre-formed cylinder booms housing signal wires that feed preamps within tip-mounted spherical sensors; (3) Extended shadow equalizers (2.5 times the sphere diameter) to mitigate photoelectron shadow mismatch for sun angles along the boom directions, particularly important at sunrise/sunset for a low inclination satellite; (4) DCcoupled electric field channels with "boosted" or pre-emphasized amplitude response at

Extended author information available on the last page of the article 
ELF frequencies; (5) Miniature multi-channel spectrum analyzers using hybrid technology; (6) Dual-channel optical lightning detector with on-board comparators and counters for 7 irradiance levels with high-time-resolution data capture; (7) Spherical Langmuir probe with Titanium Nitride-coated sensor element and guard; (8) Selectable data rates including $200 \mathrm{kbps}$ (fast), $20 \mathrm{kbps}$ (nominal), and $2 \mathrm{kbps}$ (low for real-time TDRSS communication); and (9) Highly configurable burst memory with selectable channels, sample rates and number, duration, and precursor length of bursts, chosen based on best triggering algorithm "score".

This paper describes the various sensors that constitute the VEFI experiment suite and discusses their operation during the C/NOFS mission. Examples of data are included to illustrate the performance of the different sensors in space.

Keywords Electric field detectors · Ionospheric satellite measurements · Low-latitude ionosphere

\section{Introduction}

The Communications/Navigation Outage Forecast System (C/NOFS) satellite is a US Air Force space weather mission designed to understand and forecast the ionospheric conditions that create the broad spectrum of irregularities that subsequently give rise to scintillations and outages of navigation and communication radiowave signals. With a long-term goal of forecasting such outages, the C/NOFS program included state-of-the-art instruments on a low inclination satellite, coordinated ground-based measurements, high level data processing and analysis, and theory and modeling that were combined to advance our understanding of the unstable low latitude ionosphere as well as to advance predictive capabilities.

As outlined in de La Beaujardière et al. (2004), the C/NOFS program had three scientific goals:

1. Understand the physics of the equatorial ionospheric plasma to accurately forecast the ambient ionosphere and the subsequent formation of scintillation-producing irregularities.

2. Understand the physical processes that lead to the formation of plasma depletions and instabilities in the ionosphere and identify the mechanisms that trigger or inhibit plasma instability formation.

3. Model the propagation of radiowaves through the irregular low latitude ionosphere in order to estimate the subsequent phase and amplitude scintillation.

In order to both enable this understanding and develop a reliable forecast model, accurate measurements are required. The DC electric field was determined to be among the most important drivers of both ionospheric dynamics and the creation of irregularities that create scintillations. Accordingly, the C/NOFS mission included a requirement for vector DC electric field measurements sampled at 1 sample/second (s/sec) with $0.5 \mathrm{mV} / \mathrm{m}$ accuracy with an $0.1 \mathrm{mV} / \mathrm{m}$ accuracy "goal". This specific requirement was established by the C/NOFS modeling team (Retterer et al. 2005).

In addition to the DC electric field measurements, the same electric field instrument would gather vector measurements of the AC electric field component of the irregularities that cause scintillations. Thus, the C/NOFS satellite included a combined DC and wave, or AC, electric field detector which together were designed to address the following key science objectives: 
Fig. 1 Plasma density data gathered on NASA's Atmosphere Explorer-E satellite as it pierced a series of nighttime depletions (a "spread-F" event) shown in the top panel with associated strong, backscatter radar echoes observed simultaneously in Kwajalein Atoll, shown in the bottom panel (Tsunoda et al. 1982)
AE Satellite - Plasma Density Measurements

Altair Radar (Kwajalein) - Backscatter Echoes 13 August 1978
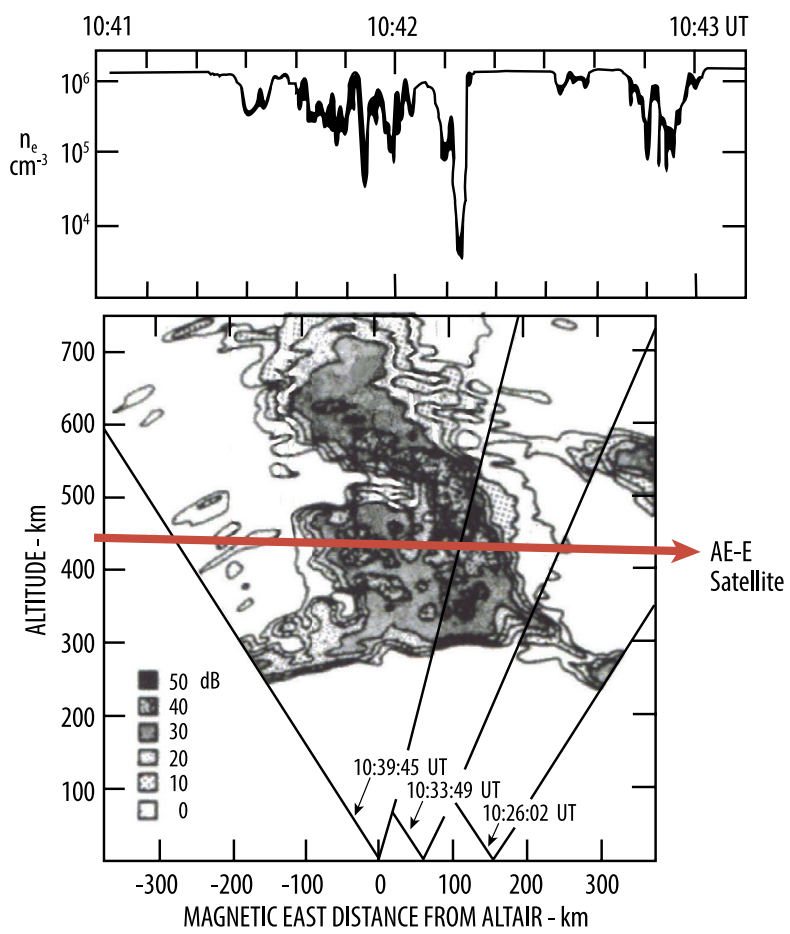

1. Investigate how the ambient DC electric field, in concert with the neutral wind and plasma density, initiate nighttime ionospheric depletions and turbulence (e.g., spread-F).

2. Characterize the quasi-DC electric fields associated with abrupt, large amplitude $\mathbf{E} \times \mathbf{B}$ drifts within depleted flux-tubes associated with irregularities.

3. Quantify the spectrum of ionosphere irregularities (e.g., spread-F) and plasma structures that are associated with the unstable, low-latitude ionosphere.

In achieving these objectives, the electric field measurements would be used to both investigate a main driver of the unstable equatorial ionosphere as well as the subsequent irregularities and plasma turbulence that are a consequence of these instabilities. Indeed, the irregularities themselves are the central agent that creates the scintillations responsible for the navigation and communication outages which are at the core of the C/NOFS mission.

Low latitude ionospheric structures and instabilities have been reviewed by numerous authors (e.g., Hysell 2000; Kudeki et al. 2007; Woodman 2009). Although a synopsis of the vast literature on this subject is beyond the scope of this article, an example of experimental data is shown to demonstrate how observations of low latitude ionospheric irregularities and "spread-F" were used as criteria for the VEFI design. Figure 1 shows an example of backscatter radar echoes from the low latitude, steerable Altair radar in Kwajalein, along with the plasma density measured simultaneously by a probe on NASA's Atmosphere Explorer-E satellite as it pierced the radar volume near $425 \mathrm{~km}$ altitude (Tsunoda et al. 1982). Note the strong plasma variations measured in situ associated with the radar mea- 
Fig. 2 Rendering of the C/NOFS satellite showing 6 electric field booms deployed

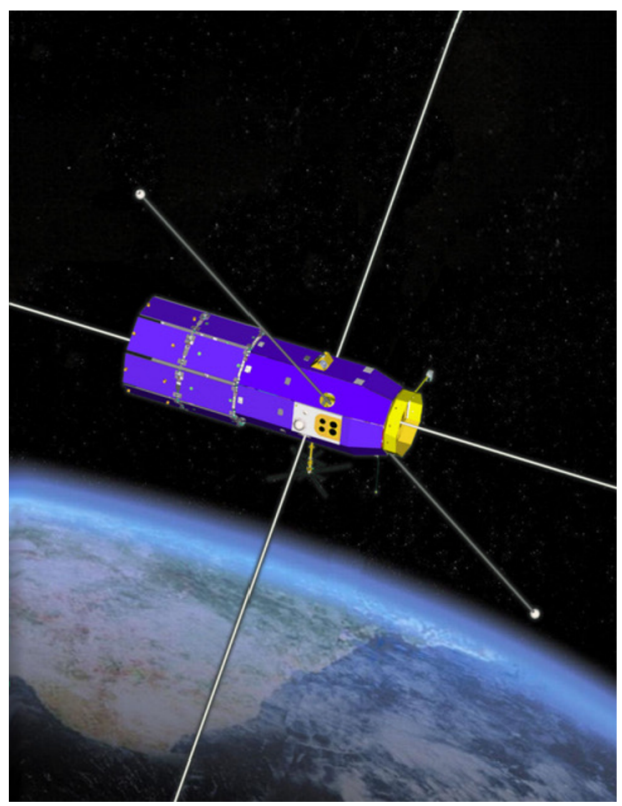

surements of ionospheric structure, and, in particular, the narrow depletion of several orders of magnitude near 10:42:12 UT that corresponds to the strongest radar echoes. The VEFI instrument was designed to measure both the very small "ambient" background DC electric fields as well as the much larger amplitude, quasi-DC fields and shorter scale irregularities within the depletions. A burst memory was thus included to trigger on the depletions (using either density variations or electric field wave amplitudes, as discussed below) and capture the high time resolution irregularities associated with the density depletions.

As the low latitude ionospheric irregularities are a nighttime phenomenon and available telemetry was limited, the VEFI measurements were subsequently designed to gather lowrate data (16 s/sec and power spectral density data) during the day and higher rate broadband and burst memory data at night. Since VEFI had a constant telemetry transfer rate to the spacecraft, the burst memory data captured during the nighttime of the previous orbit and stored in the instrument memory, were transferred to the satellite during the daytime portion of the orbit, as discussed below. In this fashion, VEFI fulfilled its objectives of measuring the DC electric fields, the quasi-DC electric fields within the depletions, as well as the high time resolution electric fields waves and irregularities associated with the depletions.

\section{$1.1 \mathrm{C} /$ NOFS Satellite Overview}

Before proceeding, we provide an overview of the C/NOFS satellite and mission which provides important context for the VEFI investigation.

The C/NOFS satellite mission was a US Air Force Research Laboratory project that was part of the US Department of Defense Space Test Program. It was managed by the Air Force Research Laboratory, originally at Hanscom AFB, MA, and now at Kirtland AFB, NM. The C/NOFS Data Center and operations were carried out initially at Hanscom AFB and later at Kirtland AFB.

The C/NOFS satellite is depicted in Fig. 2. A photograph of C/NOFS on the Pegasus vehicle is shown in Fig. 3. The satellite was designed to be $\sim 1 \mathrm{~m}$ in diameter to maximize 
Fig. 3 Photograph of the $\mathrm{C} / \mathrm{NOFS}$ satellite inside the Pegasus fairing

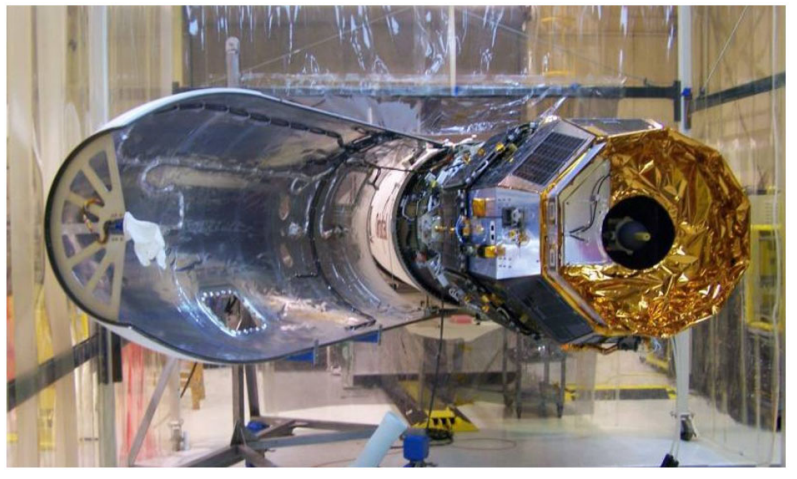

the solar array surface area while fitting within the available launch vehicle fairing envelope. The circular opening shown at the top of the satellite enables one of the electric field booms to deploy, as discussed below.

The C/NOFS spacecraft was designed, built, and tested by Spectrum Astro, of Mesa, Arizona, which later became General Dynamics. C/NOFS is a three-axis stabilized, ram/nadir oriented (non-spinning) spacecraft that included ram-pointing instruments for which three axis attitude control was maintained using a momentum wheel and magnetic torque rods. The satellite included body-mounted solar panels and a solar array skirt. The ram-pointing solar array panels were conductive. The satellite did not include propulsion. C/NOFS included redundant star sensors to provide attitude to 0.05 degree. Its total mass was $395 \mathrm{~kg}$ and the nominal mission lifetime was 1 year.

C/NOFS was launched on a Pegasus vehicle from Kwajalein Atoll on April 17, 2008 at 17:02:47 UT into a 13-degree inclination orbit with an initial perigee and apogee of $401 \mathrm{~km}$ and $867 \mathrm{~km}$, respectively. The subsequent orbital period was $102 \mathrm{~min}$. This eccentric orbit included an apsidal precession period of 64 days.

C/NOFS was launched into a period of exceptionally low solar activity, and thus the subsequent orbit decay was much more gradual than expected. Figure 4 shows the evolution of the mission apogee and perigee, and hence, the orbit decay over time. The upper panel shows the measured solar EUV measurements from the NASA TIMED satellite (Woods et al. 2005). As solar activity increased in 2011, the apogee correspondingly started to decrease more noticeably. Eventually, after the first quarter of 2015, the apogee became lower than $500 \mathrm{~km}$ with perigee at this time below $350 \mathrm{~km}$. In late November 2015, the spacecraft orbit circularized, and re-entry occurred on November 28, 2015, after approximately 7.5 years in orbit.

A 5-month pause in operations occurred in 2013, due to a shortage of funding. During this time, starting in May 2013, the spacecraft operations were put into a safe hold. Operations resumed in October 2013, in order to take advantage of the lower altitude measurements at the end of the mission.

C/NOFS included a number of ram-facing instruments, including an ion velocity meter and neutral wind meter that comprised the NASA-funded Coupled Ion Neutral Dynamics Investigation (CINDI) instrument suite provided by the University of Texas, Dallas and the Planar Langmuir Probe (PLP) provided by the Air Force Research Laboratory. These instruments required the spacecraft front face to be oriented in the ram direction within 3 degrees. Other C/NOFS instruments included the Coherent Electromagnetic Radio Tomography (CERTO) experiment provided by the Naval Research Laboratory and the C/NOFS 
C/NOFS Orbit History and Solar EUV

April 16, 2008 to November 28, 2015

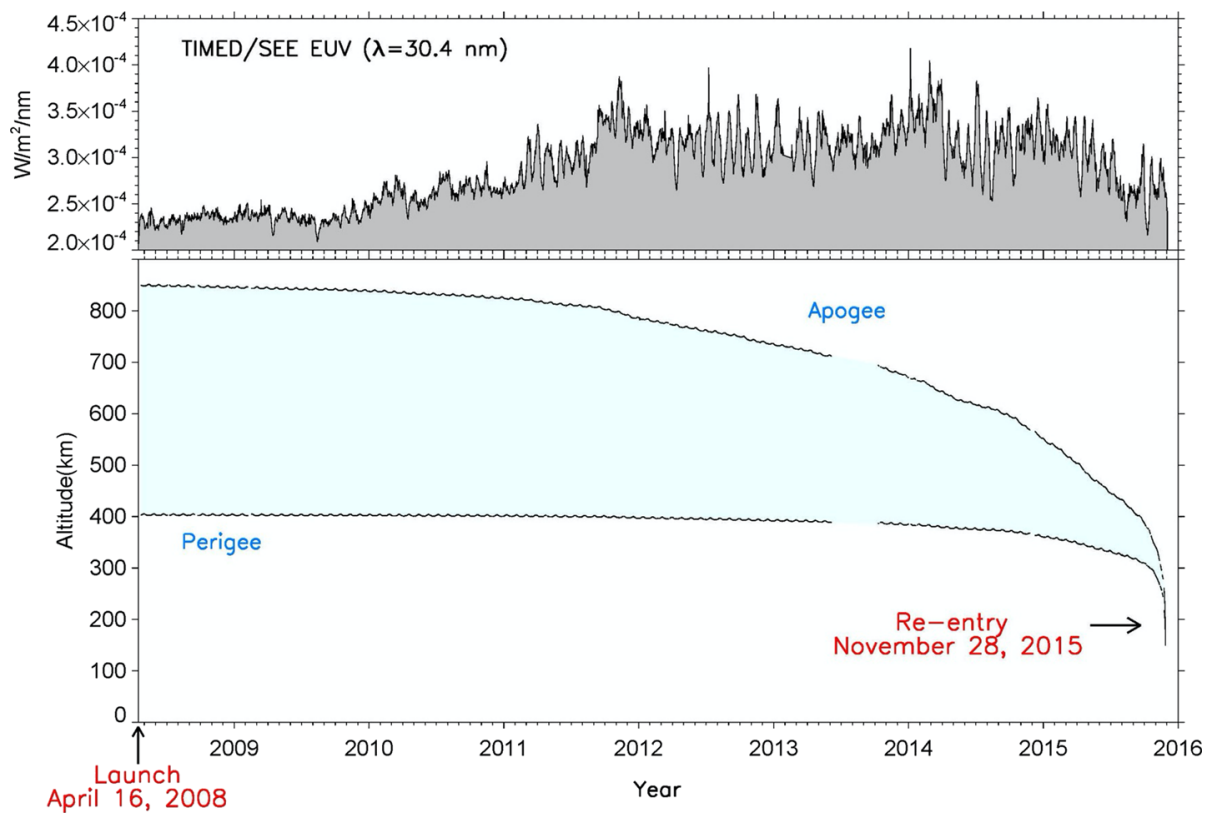

Fig. $4 \mathrm{C}$ /NOFS orbit versus time, from launch to re-entry. The upper panel shows the EUV flux measurements from the TIMED satellite, an indicator of solar activity

Occultation Receiver for Ionospheric Sensing and Specification (CORISS) experiment, provided by the Aerospace Corporation, which included a dual frequency GPS receiver to measure line-of-sight total electron content.

C/NOFS included a number of different telemetry modes, depending on the phase of its operations. These included Fast Transmit, Regular Transmit, and Forecast Transmit modes, for which the designated VEFI telemetry rates were $200 \mathrm{kbps}, 20 \mathrm{kbps}$, and $2 \mathrm{kbps}$, respectively. Regular and Fast Transmit are for use with the SGLS transmitter during normal operations. Forecast Mode consisted of low-rate data designed to be used with the NASA TDRSS constellation during the mission to provide "real time" measurements for situational awareness purposes as well as to serve as inputs to operational models that forecast navigations and communication outages at all local times and longitudes.

\subsection{VEFI Programmatic Notes}

The Vector Electric Field Investigation (VEFI) experiment was funded entirely by the US Air Force and was designed, built, and tested at the NASA/Goddard Space Flight Center, (R. Pfaff, P.I.). VEFI was funded via a reimbursable agreement between the Air Force Research Laboratory and NASA. The project at Goddard officially began (funding released) on April 1, 2001. The complete hardware investigation including all hardware engineering analyses and reviews and environmental testing at Goddard was delivered on time and on budget to the project, on March 1, 2003, less than two years later. Some instrument software upgrades were delivered later. 


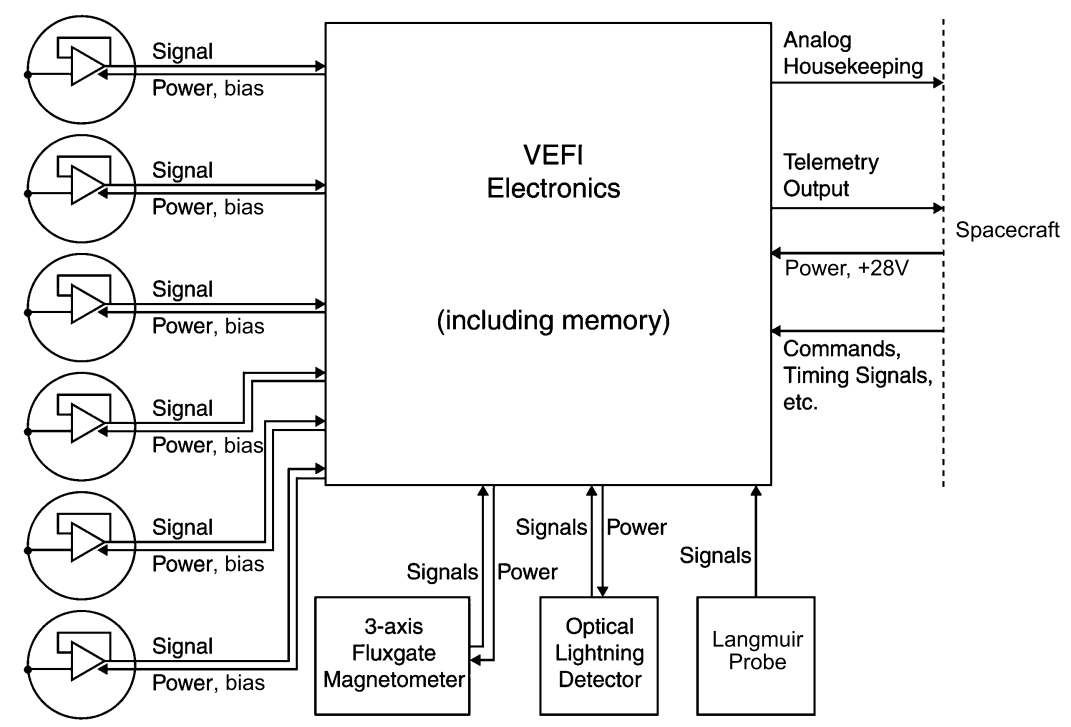

Fig. 5 Block diagram of the VEFI instrument showing different sensors that were input to the main electronics box
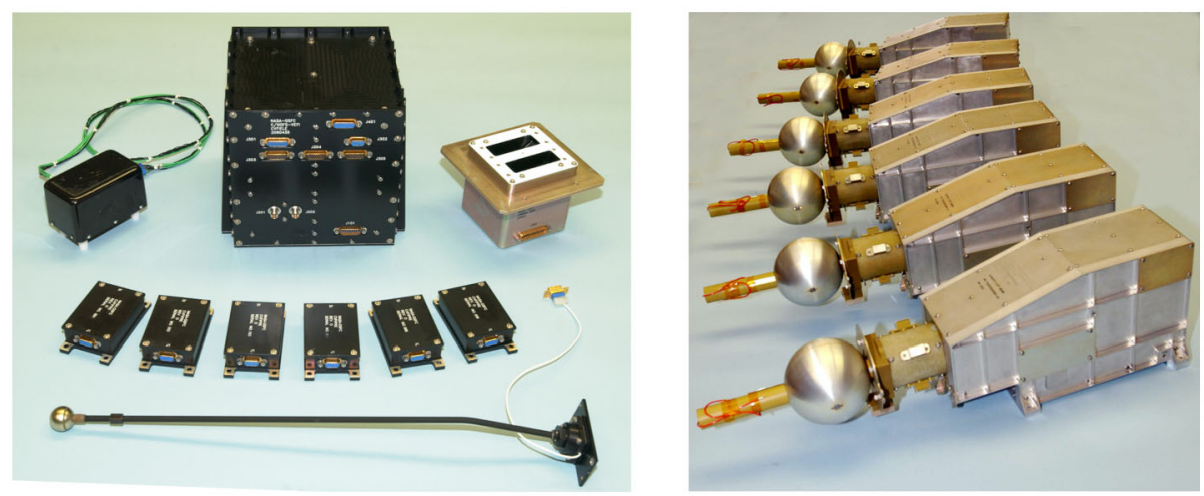

Fig. 6 Photograph of the VEFI hardware (except harnesses) provided by Goddard for the C/NOFS mission. The photograph on the left shows the main electronics box, 6 pre-amps for the cylindrical sensors, the fluxgate magnetometer, Langmuir probe, and the lightning detector. The photograph on the right shows the stowed configuration of the 6 electric field booms with spherical sensors (and embedded pre-amps)

\section{Overview of the VEFI Instrument Suite}

The Vector Electric Field Investigation (VEFI) on C/NOFS consists of one central electronics box that receives inputs from electric field double probes, a fixed-bias Langmuir probe, a flux-gate magnetometer, and a lightning detector. The VEFI instrument is thus one experiment with many sensors. A block diagram of the VEFI instrument is shown in Fig. 5. A photograph of VEFI hardware is provided in Fig. 6.

The central element of the Vector Electric Field Instrument (VEFI) investigation on the C/NOFS satellite is the DC electric field detector. Based on the well-established double- 
probe technique for ionospheric electric field measurements (e.g., Maynard 1998), VEFI extends spherical sensors with embedded pre-amps at the ends of six, 9.5-m booms that form a system of three orthogonal electric field detectors with baselines of $20 \mathrm{~m}$ tip-to-tip each. The prime VEFI measurement is the vector (3-axis) DC electric field at 16 vectors/sec with an accuracy of $0.5 \mathrm{mV} / \mathrm{m}$ and a goal of $0.1 \mathrm{mV} / \mathrm{m}$. The main data product delivered by VEFI to the C/NOFS AFRL Data Center is the vector DC electric field at 1 vector/sec which was an average of the $16 \mathrm{~s} / \mathrm{sec}$ data that were telemetered to the ground.

In order to help achieve the required accuracy, Goddard also provided a sensitive fluxgate magnetometer as part of VEFI to measure the earth's magnetic field and its variations. Including this measurement ensured that the contribution of $\mathbf{V} \times \mathbf{B}$ fields to the electric field measurements were accurately ascertained and removed and enabled accurate $\mathbf{E} \times \mathbf{B}$ drifts to be computed from the electric field measurements. This was particularly important in the region of the South Atlantic Anomaly where the model magnetic field was considered to be less accurate, particularly during the epoch of the late 1990's when the C/NOFS project was conceived. The same magnetometer data also revealed important magnetic field structures and currents inherent to the low latitude ionosphere. Vector magnetic field data were provided at 1 vector/sec with an AC-coupled magnetic field measurement provided at 16 vector/sec, as discussed below.

In addition to the DC electric and magnetic field data, VEFI also measures high time resolution wave data consisting of broadband ELF electric field data at $512 \mathrm{~s} / \mathrm{sec}$ routinely gathered during nighttime passes (this rate could be increased to $8192 \mathrm{~s} / \mathrm{sec}$ for special fast telemetry mode) as well as burst memory snapshots up to $32 \mathrm{ks} / \mathrm{sec}$. In addition, VEFI continuously calculated on board power spectra in the VLF and HF frequency domains. In this manner, VEFI captures the broad spectra of electric field irregularities associated with equatorial spread-F and related ionospheric processes as well as a wide variety of other plasma waves and structures.

A simple "fixed-bias" spherical Langmuir Probe was also provided by the Goddard team as part of the VEFI instrument suite as a means to provide input to the VEFI burst memory when the spacecraft encountered plasma depletions, thus triggering the collection of high time resolution vector wave measurements. This probe also served as a backup density measurement to the satellite's dedicated Planar Langmuir Probe (Roddy et al. 2010). The VEFI Langmuir probe provided continuous relative plasma density measurements at $16 \mathrm{~s} / \mathrm{sec}$ as well as high time resolution plasma density waves or irregularities at $512 \mathrm{~s} / \mathrm{sec}$ in the ELF mode. Burst memory snippets of the plasma density extended the sample rate to $32 \mathrm{k} / \mathrm{sec}$.

Finally, a lightning detector was added to the Goddard instrument suite as a means to help distinguish the contribution of lightning-related electric fields to instability and wave formation within the low latitude ionosphere. The lightning detector design and prototype were provided by Drs. R. Holzworth and M. McCarthy of the University of Washington.

As described herein, VEFI consisted of the following sensors and data products, all constituting one overall investigation:

- DC and AC Electric field detectors:

- DC electric fields in a range of $\pm 450 \mathrm{mV} / \mathrm{m}$ at 16 vectors $/ \mathrm{sec}$

- Time-domain quasi-DC electric field structures

- Plasma waves and irregularities in broadband time series for two channels sampled at $512 \mathrm{~s} / \mathrm{sec}$, with up to 8192 /sec continuous sampling in high telemetry mode

- On-board VLF FFT spectrograms $(0-16 \mathrm{kHz})$

- On-board HF FFT spectrograms (0-4 MHz)

- Snapshots of DC-coupled and VLF data up to $32 \mathrm{ks} / \mathrm{sec}$ 
Table 1 VEFI data channels showing their accuracy, dynamic range, sampling frequency, and main data focus

\begin{tabular}{|c|c|c|c|c|c|}
\hline $\begin{array}{c}\text { Measurement } \\
\text { Output }\end{array}$ & Units & $\begin{array}{l}\text { Estimated } \\
\text { Accuracy }\end{array}$ & Sample Frequency & $\begin{array}{c}\text { Output } \\
\text { range }\end{array}$ & Main Focus for Data \\
\hline $\begin{array}{c}\text { DC Electric Field } \\
(1 \mathrm{~s} / \mathrm{sec})\end{array}$ & $\mathrm{mV} / \mathrm{m}$ & $0.5 \mathrm{mV} / \mathrm{m}$ & $\begin{array}{c}1 \text { sample/sec } \\
\text { (3 components) }\end{array}$ & $\begin{array}{c} \pm 450 \mathrm{mV} / \mathrm{m} \\
\text { prior to } \mathbf{V} \times \mathbf{B} \\
\text { removal }\end{array}$ & $\begin{array}{ll}\text { - } & \text { Large scale ESF drivers } \\
\text { - } & \text { Large scale electrodynamics } \\
\text { - } & \text { Gravity waves } \\
\text { - } & \text { Integrated potential along orbit }\end{array}$ \\
\hline $\begin{array}{l}\text { DC Electric Field } \\
(16 \mathrm{~s} / \mathrm{sec})\end{array}$ & $\mathrm{mV} / \mathrm{m}$ & $0.5 \mathrm{mV} / \mathrm{m}$ & $\begin{array}{l}16 \text { sample } / \mathrm{sec} \\
(3 \text { components })\end{array}$ & $\begin{array}{c} \pm 450 \mathrm{mV} / \mathrm{m} \\
\text { prior to } \mathbf{V} \times \mathbf{B} \\
\text { removal }\end{array}$ & $\begin{array}{l}\text { - High spatial resolution }(0.5 \mathrm{~km}) \\
\text { Electrodynamics } \\
\text { ESF depletion physics, } \mathrm{km} \text { scale } \\
\text { instabilities, Alfven waves }\end{array}$ \\
\hline $\begin{array}{l}\text { AC Electric Field } \\
\text { Waveforms }\end{array}$ & $\mathrm{mV} / \mathrm{m}$ & $\begin{array}{c}0.01 \\
\mathrm{mV} / \mathrm{m}\end{array}$ & $\begin{array}{c}512 \mathrm{~s} / \mathrm{sec} \text { (nominal) } \\
2048,4096,8192 \mathrm{~s} / \mathrm{sec} \\
\text { (Fast survey) }\end{array}$ & $\pm 45 \mathrm{mV} / \mathrm{m}$ & $\begin{array}{ll}\text { - } & \text { Ionospheric Instabilities } \\
\text { - ESF irregularities, spectra }\end{array}$ \\
\hline \begin{tabular}{|c|} 
AC Electric Field \\
VLF Spectrograms \\
$(0-16 \mathrm{kHz})$ \\
\end{tabular} & $(\mathrm{mV} / \mathrm{m})^{2} / \mathrm{Hz}$ & $-80 \mathrm{~dB}$ & $\begin{array}{c}1 \text { spectrum/2 sec } \\
\text { (nominal survey), } 64 \\
\text { spec/sec (fast survey) }\end{array}$ & $100 \mathrm{~dB}$ & $\begin{array}{ll} & \text { Irregularity } \Delta \mathrm{E} \text { spectra } \\
\text { - } & \text { Instability Physics } \\
\text { - } & \text { Other wave modes } \\
\end{array}$ \\
\hline $\begin{array}{c}\text { AC Electric Field } \\
\text { Filter Bank } \\
(3 \mathrm{~Hz}-8 \mathrm{kHz})\end{array}$ & $(\mathrm{mV} / \mathrm{m})^{2} / \mathrm{Hz}$ & $-80 \mathrm{~dB}$ & $\begin{array}{c}1 \text { spectrum every } 0.75 \\
\mathrm{sec}\end{array}$ & $100 \mathrm{~dB}$ & $\begin{array}{l}\text { - Irregularity "Snapshots" for } \\
\text { real-time space weather info }\end{array}$ \\
\hline $\begin{array}{c}\text { Relative Plasma } \\
\text { Density }\end{array}$ & $\mathrm{cm}^{-3}$ & $\pm 5 \%$ & 16 samples/sec & $10^{2}-10^{7} \mathrm{~cm}^{-3}$ & $\begin{array}{l}-\quad \text { Physics of plasma depletions } \\
\text { and km-scale instabilities }\end{array}$ \\
\hline $\begin{array}{c}\text { Plasma Density } \\
\text { Fluctuation } \\
\text { Waveform }\end{array}$ & $\mathrm{cm}^{-3}$ & $\begin{array}{c} \pm 0.05 \% \\
\text { (relative) }\end{array}$ & $\begin{array}{c}512 \mathrm{~s} / \mathrm{sec} \text { (nominal) } \\
2048,4096,8192 \mathrm{~s} / \mathrm{sec} \\
\text { (Fast) }\end{array}$ & $10^{2}-10^{7} \mathrm{~cm}^{-3}$ & $\begin{array}{l}\text { - Irregularity } \Delta \mathrm{N} \text { Spectra } \\
\text { - } \Delta \mathrm{E} / \Delta \mathrm{N} \text { comparison } \\
\end{array}$ \\
\hline $\begin{array}{c}\text { DC Magnetic Fields } \\
(1 \mathrm{~s} / \mathrm{sec})\end{array}$ & nT & $\begin{array}{c}50 \mathrm{nT} \\
\text { (absolute) } \\
5 \mathrm{nT} \\
\text { (relative) }\end{array}$ & $\begin{array}{c}1 \text { sample/sec } \\
(3 \text { component vector) }\end{array}$ & $\pm 45,000 \mathrm{nT}$ & $\begin{array}{l}\text { - } \quad \mathbf{~ G e o m a g n e t i c ~ c u r r e n t s ~} \\
\text { - } \times \mathbf{B} \text { determination }\end{array}$ \\
\hline \begin{tabular}{|c|}
$\begin{array}{c}\text { AC Magnetic Fields } \\
(0.05-8 \mathrm{~Hz})\end{array}$ \\
\end{tabular} & nT & $0.1 \mathrm{nT}$ & $\begin{array}{c}16 \mathrm{~s} / \mathrm{sec} \\
(3 \text { component vector }) \\
\end{array}$ & $\pm 900 \mathrm{nT}$ & $\begin{array}{l}\text { - ESF Magnetic perturbations } \\
\text { - } \quad \text { Poynting flux, Alfven waves } \\
\end{array}$ \\
\hline $\begin{array}{c}\text { Optical Lightning } \\
\text { Detector Levels }\end{array}$ & Counts & & $\begin{array}{c}\text { Count rates in } 7 \text { bins } \\
\text { twice per sec in North, } \\
\text { South directions }\end{array}$ & & $\begin{array}{l}\text { - Correlate storms with ESF } \\
\text { - Thunderstorm related electric } \\
\text { fields } \\
\text { - Causal link for explosive ESF }\end{array}$ \\
\hline $\begin{array}{l}\text { Burst Memory } \\
\text { (all VEFI data) }\end{array}$ & Varied & & $\begin{array}{l}1-8 \text { channels } \\
\text { up to } 32 \mathrm{k} \mathrm{s} / \mathrm{sec} \text { per } \\
\text { channel. }\end{array}$ & & $\begin{array}{ll} & \text { Full ESF instability spectra } \\
\text { - } & \text { Interferometry } \\
\text { - } & \text { Lightning generated E fields }\end{array}$ \\
\hline
\end{tabular}

- Magnetometer

- DC magnetic fields at 1 vector/sec in $\pm 45,000 \mathrm{nT}$ range

- AC magnetic fields at 16 vectors/sec in $\pm 900 \mathrm{nT}$ range

- Fixed-bias Langmuir Probe (Trigger Probe)

- Relative Plasma Density (from $10^{2}$ to $10^{7} \mathrm{~cm}^{-3}$ ) and $\Delta \mathrm{N} / \mathrm{N}$ fluctuations

- Lightning Detector (w/Univ. of Washington)

- Count rates of optical lightning irradiance "flashes" within 7 amplitude levels in north and south directions

- Burst memory

- 1-8 selectable channels sampled at up to $32 \mathrm{kHz} / \mathrm{channel}$

- Burst data could be either DC-coupled or AC-coupled electric field data and/or magnetic field data, plasma density data (DC or AC coupled) or optical lightning detector waveforms

Table 1 lists the VEFI instrument performance and data products.

A photograph of the C/NOFS satellite during spacecraft integration is shown in Fig. 7. The folded solar array skirt that extends from the base of the spacecraft is in the stowed configuration and is not visible here. The photograph shows two of the electric field booms 
Fig. 7 Photograph of the $\mathrm{C} / \mathrm{NOFS}$ satellite with experiments installed at General Dynamics prior to delivery to the launch vehicle

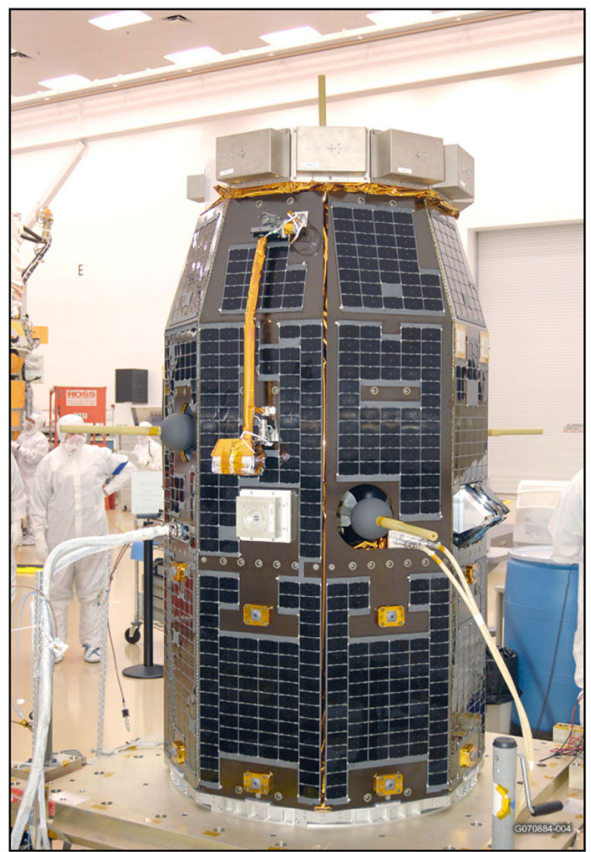

deployed a very short distance, revealing their carbon-coated electric field spherical sensors and deployed shadow equalizers. The VEFI magnetometer is in the center left of the photograph. It is covered by a gold-colored thermal wrap at the end of its boom, shown stowed against the side of the spacecraft. A diagram showing the C/NOFS satellite with its deployed sensor configuration is provided in Fig. 8. This diagram shows the orientation of the instruments with respect to the spacecraft velocity and nadir directions as well as the approximate orientation of the ambient magnetic field, $\mathbf{B}$, throughout the mission.

The main VEFI electronics consists of differential amplifiers, Langmuir probe circuitry, filters, 16-bit A/D converters, an 8 Mbyte memory including burst capabilities, and microprocessors that execute, among other tasks, a triggered "snapshot" data collection scheme and an FFT algorithm. The electronics represent an evolved heritage based on past Goddard electric field instruments on low earth-orbiting satellites such as Dynamics Explorer-2 and San Marco, launched in 1981 and 1988, respectively, as well as DC/AC Goddard-led electric field experiments flown on over 50 sounding rocket missions during the previous 30 years.

The overall VEFI mass was $54 \mathrm{~kg}$, largely due to the six electric field booms and sensors which had a mass of $7 \mathrm{~kg}$ each. The overall VEFI power was 11.8 Watts. A low power mode consisted of 8.7 Watts, in which the on-board processing and burst memory were disabled.

The VEFI electronics gathers telemetry in one of four modes from the VEFI instruments: Slow Survey ( $\sim 2 \mathrm{kbits} / \mathrm{s})$, Nominal Survey $(\sim 20 \mathrm{kbits} / \mathrm{s})$, Fast Survey $(\sim 200 \mathrm{kbits} / \mathrm{s})$, and burst mode. Data is transmitted to the spacecraft from the VEFI electronics in one of three modes corresponding to the satellite specified collection modes: Regular Transmit (Nominal Survey, $20 \mathrm{kbits} / \mathrm{s}$ or Slow Survey + Burst Downlink), Fast Transmit (200 kbits/s), and Forecast ( $2 \mathrm{kbits} / \mathrm{s}$ ) as described below.

As discussed above, since the low-latitude ionospheric irregularities are a nighttime phenomenon, the VEFI measurements were designed to gather low-rate data $(16 \mathrm{~s} / \mathrm{sec}$ and power spectral density data) during the day and higher rate broadband and burst memory 


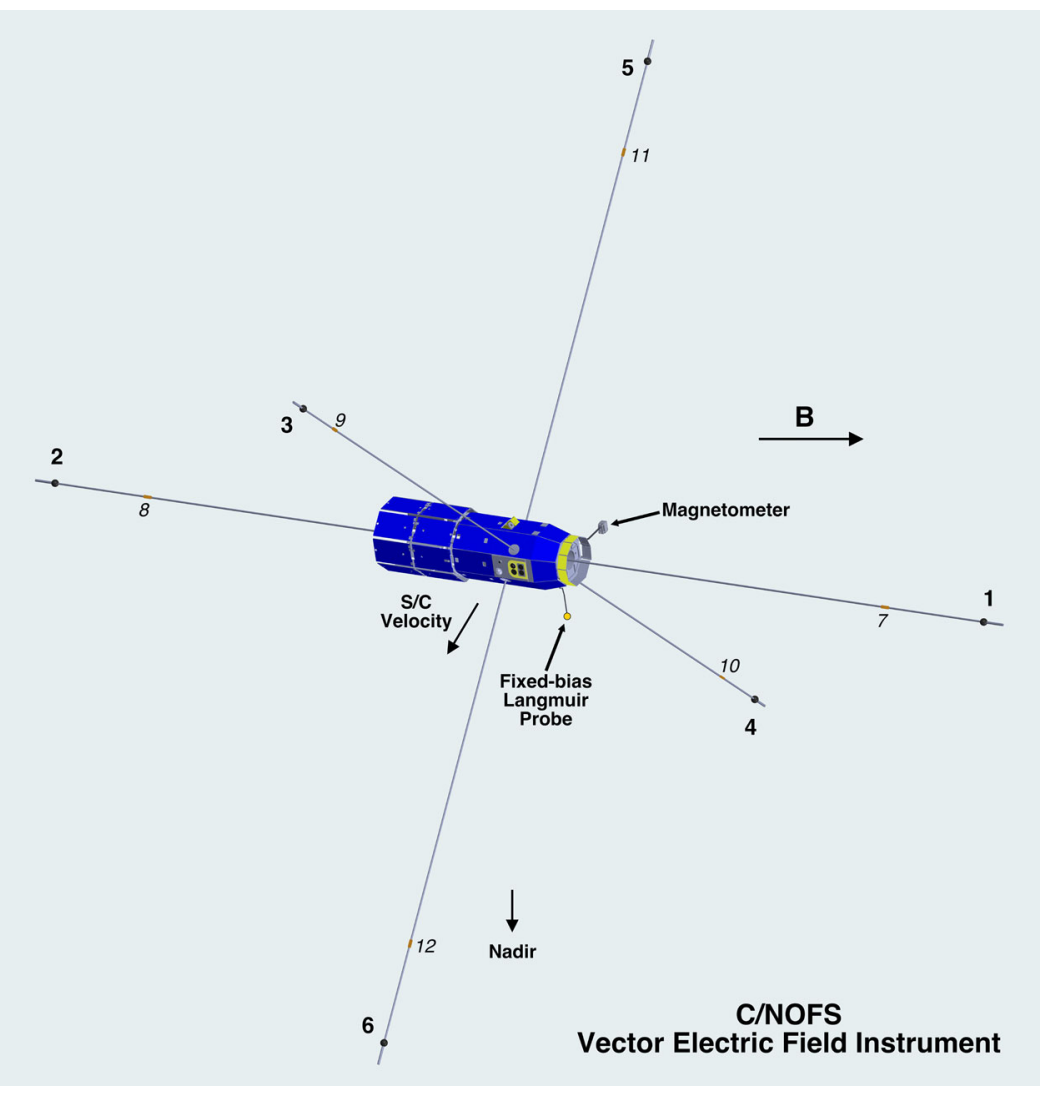

Fig. 8 C/NOFS satellite with deployed VEFI electric field booms showing flight geometry and sensor notation. Spherical sensors are numbered 1-6 whereas cylindrical sensors are numbered 7-12

data at night. Since VEFI had a constant telemetry transfer rate $(20 \mathrm{kbps})$ to the spacecraft during regular operations (day and night), the burst memory data captured during the nighttime portion of the orbit are stored in the instrument until the following daytime portion of the orbit, when they are then transferred to the satellite, as depicted in Fig. 9 and discussed further below.

In subsequent sections, we describe the individual components of the VEFI instrument suite, which were controlled by the main VEFI electronics. We also discuss the operation of the sensors during the C/NOFS mission and include examples of measurements to illustrate their performance.

\section{Vector Electric Field Detector}

VEFI's electric field detector was composed of booms, sensors, pre-amps, and a central electronics box. We discuss each element of the electric field instrument below including the analog and digital parts of the electronics. The VEFI burst memory, which included input from the magnetometer, fixed-bias Langmuir probe, and lightning detector, is discussed in a 
Fig. 9 Diagram showing the low inclination, low altitude C/NOFS orbit that included continuous data acquisition in both daytime and nighttime conditions on every orbit. VEFI was configured to acquire nominal survey data during the nightime and slow survey data during the daytime

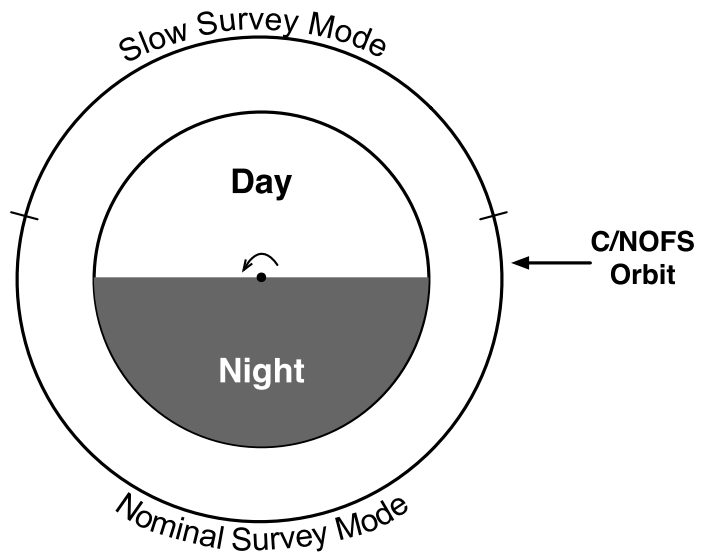

Fig. 10 Electric field double probe measurement concept showing how the potential difference of separated sensors along a baseline, $\mathrm{d}$, provide one component of the electric field

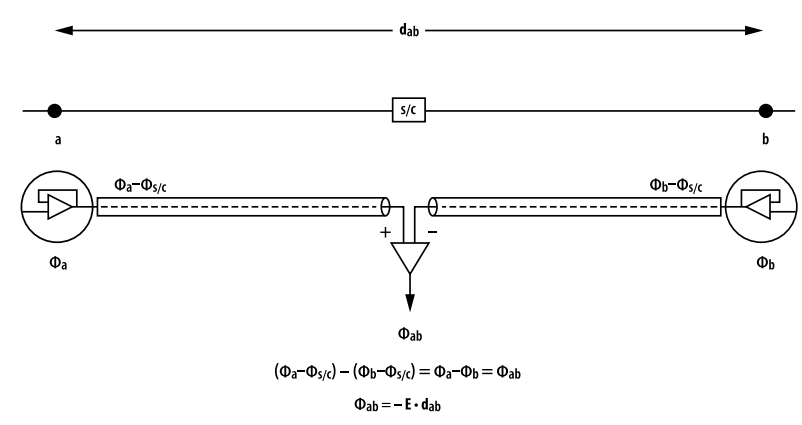

separate section. Representative examples of the DC and wave measurements are provided towards the end of the article.

\subsection{Double Probe Electric Field Detectors}

The Vector Electric Field Investigation (VEFI) on the C/NOFS satellite consists of a DC electric field detector that extends $11.8 \mathrm{~cm}$ diameter spherical sensors with embedded preamps at the ends of six, $9.5-\mathrm{m}$ booms to form a system of three orthogonal electric field detectors with baselines of $20 \mathrm{~m}$ tip-to-tip each. The measurement is based on the wellestablished double-probe technique for determining accurate, vector electric fields in the ionosphere (e.g., Fahleson 1967; Mozer 1971; Pfaff 1996; Maynard 1998) which is briefly reviewed here.

The electric field double probe is, in principle, a voltmeter which measures the electric potential between two electrodes separated in space along a separation vector, $\mathbf{d}$. Since in the absence of $\partial B / \partial t, \mathbf{E}=-\nabla \Phi$, the potential difference, $\Phi_{a b}$, measured between electrodes $a$ and $b$ (see Fig. 10) is:

$$
\Phi_{a b}=-\mathbf{E}^{\prime} \cdot \mathbf{d}+\Phi_{\text {inst }}
$$

Where $\mathbf{E}^{\prime}$ is the electric field in the spacecraft frame and $\Phi_{\text {inst }}$ represents non-geophysical contributions, including contact potentials due to work function differences of the sensor surfaces, an imbalance of photoelectron currents, spacecraft sheath effects, and other effects. 


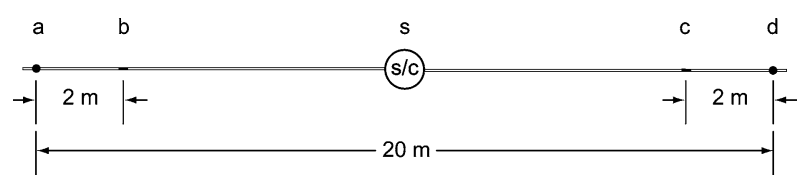

Fig. 11 Electric field sensor pair on C/NOFS showing the basic $20 \mathrm{~m}$ tip-to-tip electric field double probe. Cylindrical sensors inboard of the outboard spheres are also shown, providing a $16 \mathrm{~m}$ tip-to-tip co-linear double probe measurement

$\mathbf{E}^{\prime}$ may be related to an Earth-fixed frame of reference via

$$
\mathbf{E}^{\prime}=\mathbf{E}_{o}+\mathbf{V}_{s / c} \times \mathbf{B}
$$

where $\mathbf{E}_{o}$ is the ambient electric field in the Earth-fixed frame and $\mathbf{V}_{s / c} \times \mathbf{B}$ is the electric field generated by the motion of the probe, $\mathbf{V}_{s / c}$, measured relative to the Earth-fixed frame, across the magnetic field, $\mathbf{B}$.

It is clear from (1) that the measured signal due to the electric field increases with the probe separation distance (or the boom length), whereas the non-geophysical contributions do not. In fact, in the ionosphere, some of the non-geophysical contributions due to spacecraft sheath, photoelectron imbalance, wake effects, etc., may be expected to decrease with increasing distance from the spacecraft. Thus, increasing the signal/noise ratio is the primary reason why double probe experiments require long measurement baselines. Other reasons include minimizing shadow effects from the spacecraft body as well as extending the sensors beyond any spacecraft-generated potentials and other sources of interference. By gathering simultaneous difference potentials in three orthogonal directions a measurement of the vector electric field in the spacecraft frame is obtained. With suitably accurate inertial attitude and velocity knowledge, the vector electric field can be transformed into the geophysically relevant frame.

The actual measurement of the potential difference by the double probe is somewhat more complicated since any conducting surface immersed in a plasma acquires a net charge. In essence, what is measured is the floating potential of each probe with respect to the plasma potential, and these measurements are referenced with respect to the spacecraft potential. For a potential difference measurement acquired with identical symmetric probes, the spacecraft potential does not contribute, as shown in Fig. 10. Variations that might exist in the plasma potential due to naturally occurring (geophysical) plasma density and temperature gradients between the two sensor locations have been shown to be inconsequential $(\ll 1 \%)$ in the ionosphere (Laakso et al. 1995).

A diagram of one pair of opposing VEFI boom elements on C/NOFS showing symmetric sensor double probes is shown in Fig. 11. Both spherical sensors at the boom ends and cylindrical sensors inboard are shown. We return to the inner cylindrical sensors below.

\subsection{Electric Field Measurement Geometry on C/NOFS}

The booms that comprise the VEFI electric field detector are oriented to provide three orthogonal 20-meter tip-to-tip double probes that are used to detect the vector DC and AC electric field. The geometry of the vector measurements and sensor numbering is shown in Fig. 8. (See also Fig. 42.)

Importantly, the VEFI electric field configuration on C/NOFS consists of two orthogonal double probes in the orbit plane. The main reason for choosing this configuration is the 
low inclination of the C/NOFS orbit (13 degrees). At this low inclination, the earth's ambient magnetic field is aligned predominantly perpendicular to the orbit plane throughout the mission at all altitudes. Accordingly, using the very good assumption that there are no DC electric fields along (or parallel to) the direction of the ambient magnetic field, for the low latitude, low altitude plasma environment of C/NOFS, the two measured components of the DC electric field in the orbital plane provide a very good measure of the vector electric field, provided the sphere locations are known. Indeed, this was precisely how vector electric fields were measured by the low-inclination (spinning) San Marco satellite using its two 40 m tip-to-tip spin plane double probes (Maynard et al. 1995). In the case of C/NOFS, the third orthogonal axis, which is perpendicular to the orbital plane, is used to increase the accuracy of the DC electric field measurements, providing a precise determination of the electric field offsets and enabling the on-orbit non-orthogonality matrix of the electric field sensor configuration to be determined with high precision. The complete, tri-axial electric field data also provided unprecedented measurements of wave electric fields that included the component parallel to the magnetic field, which is important for some wave modes, such those associated with lightning. An example of AC electric fields measured along the magnetic field direction with the C/NOFS VEFI instrument is shown further on below (see Fig. 78).

Another reason why the two orthogonal double probes were placed in the orbit plane on $\mathrm{C} / \mathrm{NOFS}$ is that since this plane primarily included the electric fields that were the main focus of the VEFI experiment, the corresponding four spherical sensors provide alternative potential difference measurements within the orbit plane. In other words, a second set of differentials within the orbital plane is available from double probe measurements created from adjacent segments that include the "corners" of the crossed double probes. Such measurements, though less accurate, provided a check of the main electric field solution as well as offer some redundancy in the event of possible sensor degradation that might occur during an extended mission.

From the standpoint of the spacecraft moment of inertia and small forces which may be imparted to the booms from, say, neutral density variations, another advantage of having four booms placed symmetrically within the orbit plane is that this plane is co-planar with that of the momentum wheels in the spacecraft which are used to maintain the spacecraft orientation and three-axis pointing. Slight forcing on these booms from variations of the ambient neutral density encountered by the spacecraft, for example, would be introduced in the same plane where the momentum wheels are best able to maintain the spacecraft pointing.

Furthermore, the C/NOFS satellite was periodically spun about the orbit normal (the axis parallel to the "1-2" double probe axis) to enable electric field and magnetic field offsets to be checked, as well as to characterize the performance of the forward-pointing instruments that measure gas properties at "off ram" angles. Having four booms (two orthogonal double probes) in the orbit plane provided the optimum geometry for these periodic spacecraft spin tests (see example below), for both reasons of moment of inertia during the spin and as well as to provide independent offsets of the two principal orthogonal electric and magnetic field components within that plane.

Finally, having the booms oriented in the orbit plane with crossed booms at 45 degrees to the ram and zenith minimized upward and downward obstructions to the field-of-view of other instruments, such as the CERTO beacon antenna, the star sensors, and the VEFI lightning detector. This geometry also avoided any electric field measurement directly in the spacecraft wake. 


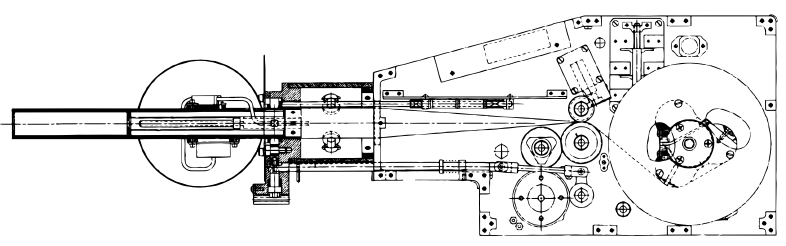

Fig. 12 Drawing of the deployer mechanism showing how the pre-formed cylindrical antenna is flattened as it is coiled about the main spool, as shown on the right. The left hand side shows the caged sphere at the end of the cylinder in the stowed configuration
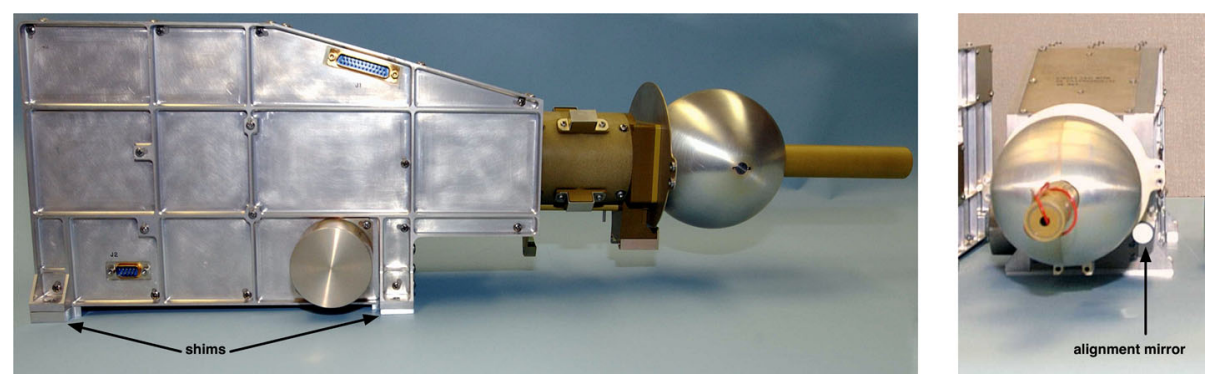

Fig. 13 Photographs of a VEFI electric field deployer showing a side view (left) and front view (right). The side view shows the shims that were placed on the mounting feet to optimize the orthogonal measurements after deployment. The front view shows the alignment mirror used to measure the positions of the boom deployers mounted in the spacecraft to provide an initial orthogonality matrix

\subsection{Electric Field Booms and Sensors}

The VEFI electric field booms are similar to those originally developed by Fairchild Space (later Orbital Sciences Corp.) that were flown on the Dynamics Explorer-2 (DE-2) satellite (Maynard et al. 1981) as well as on the Cassini satellite (Gurnett et al. 2004). In the case of the C/NOFS electric field experiment, spherical sensors with embedded pre-amps were placed at the ends of the booms and connected to the central electronics with wires down the center of the booms. Shadow equalizers extend past the spheres to minimize photoelectron current imbalances of opposing spheres due to asymmetric shadowing of opposing spheres by the boom elements, as described below. A caging mechanism was added to capture the sphere from behind, at its base, and was released via a rod that was activated when the boom was released. A mechanical diagram of the deployer is shown in Fig. 12. A photograph of a VEFI boom deployer unit is shown in Fig. 13.

The VEFI boom elements are composed of two, semi-circular Beryllium Copper elements that are joined via tab and slots along their edges, forming a $2.86 \mathrm{~cm}$ diameter cylindrical element when deployed. The elements were heat-treated in order to maintain their shape after they are deployed, after having been stored in a flattened form, coiled on a spool. When deployed, they form a rigid cylinder that provides stable positioning of the spherical sensor at the end.

The elements are continuously perforated along their length (12\% hole area) to equalize thermal gradients caused by solar illumination, and have thermal control coatings (silvered on the outside with an outer layer of Teflon to protect the silver from oxidation, black paint on the inside), to equalize the temperature between hot and cold sides (see Fig. 3 of Maynard et al. 1981). This design minimizes the thermal-induced deflection of the booms, allowing 
Fig. 14 Close up of a segment of the cylindrical boom element with ribbon of conductors in the interior and connector

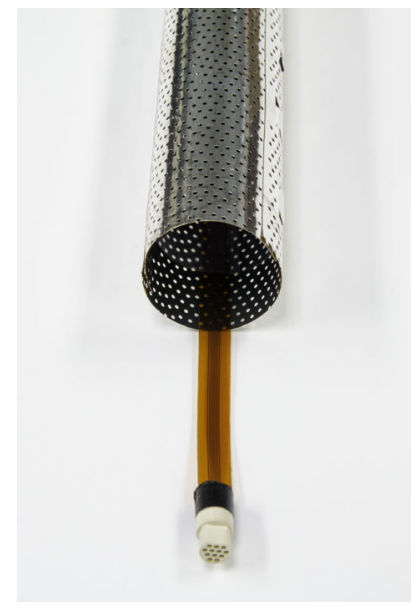

accurate measurements in both illuminated and eclipse conditions. These are the same thermal properties successfully implemented on similar booms flown in the ionosphere on the Dynamics Explorer-2 satellite.

The VEFI booms were originally provided by Orbital Sciences Corp. (formerly Fairchild Space) to AFRL in the late 1980's for the Interactive Measurements Payload for Shuttle (IMPS) program, which was later cancelled. The six booms of $12 \mathrm{~m}$ length were then transferred to NASA/Goddard Space Flight Center in May 2001, where they underwent initial testing followed by refurbishment, modifications, and testing at Orbital under contract and direction from the VEFI team at NASA/Goddard.

The VEFI booms were modified from the DE-2 design to include a sphere at their ends and to deploy a signal wire and feed wires down the length of the boom element to connect the pre-amp with the main VEFI electronics. Using the existing elements and allowing for volume growth due to the internal wires, the length of the modified boom elements was reduced to $9.5 \mathrm{~m}$ measured from exit aperture at the boom base to the center of sphere. Later, the original boom elements delivered as part of the IMPS hardware were replaced with new ones.

The thin ribbon cable placed down the center of each boom, as shown in Fig. 14, included 8 conductors. Pull tests and other precautions were taken to ensure that the wire would not break or bind during deployment/or retraction.

Other refurbishment/modification of the IMPS booms entailed redesigning the storage reel assembly and side plates to add electrical contact slip rings, adding a rotating coax connector and signal wire connector, redesigning the new drive roller assembly, resizing the storage reel bearing, redesigning a new forward section of the mechanism to support and cage the new sphere assembly, adding a cage and linkage to prevent self-deployment of the boom and sphere, and adding element guide rollers for the boom alignment. An alignment mirror was added to each boom and a custom shim was provided for each boom to account for any inherent misalignment or boom bending within the $9.5 \mathrm{~m}$ length as determined during testing.

A caging device was implemented on each boom to capture the sphere from behind during launch, in order to prevent a partial, premature deployment. When the caging device was released in orbit, a short $(12 \mathrm{~cm})$ tubular element was simultaneously released outward of the sphere to extend the shadow equalizer, as shown in Fig. 15. 


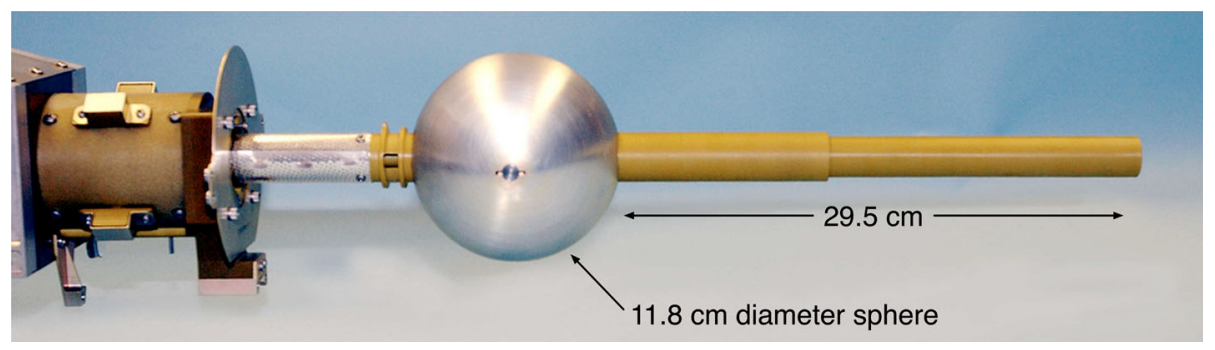

Fig. 15 Sphere at the end of the boom element with fully deployed shadow equalizer

Each boom was deployed using a brushless DC motor using a +28 Volt $( \pm 6 \mathrm{~V})$ supply. The booms included limit switches and other monitors to determine the boom length to within $0.1 \%$. The boom extension was designed to stop when the booms reached the end of their deployment (or retract) cycle, or when power was no longer applied. A dedicated custom boom deployment electronics box was designed and built for deploying the booms. This was provided by the spacecraft manufacturer. Boom housekeeping data, such as the length indicator and limit switch status, were provided via the main telemetry.

The booms were designed to deploy (and retract, if necessary) on command. In orbit, after all 6 booms were uncaged, the booms in the orbit plane (the " $3-4$ " and "5-6" booms) were deployed as pairs, initially to $1 \mathrm{~m}$ length, then to $5 \mathrm{~m}$, and finally to full length. Data were gathered for a full day (15 orbits) at each of these intermediate stations to provide unique information pertaining to the plasma environment and electric fields near the spacecraft. After another 3 days, the "1-2" booms were deployed, again to $1 \mathrm{~m}, 5 \mathrm{~m}$, and then full deployment, with a full day between stations. VEFI boom deployment was fully accomplished 21 days after launch.

Each of the VEFI boom elements was insulated from the space environment, with the exception of a short segment $(0.25 \mathrm{~m})$ on each boom that was made available as a sensor element. This short segment was situated $2 \mathrm{~m}$ inboard, measured from the center of the sphere, as shown in Fig. 11. As successfully demonstrated for the DE-2 booms, the insulation consisted of a Teflon coating which served to keep the silver coating of the $\mathrm{BeCu}$, included for thermal reasons, from oxidizing.

Spacecraft attitude and control design required accurate representation of the deployed boom characteristics. Engineering tests for each boom were conducted to determine: (1) the resonance of the boom plus tip mass, (2) boom damping characteristics, (3) boom bending EI and root stiffness. Computer analysis of the boom characteristics was also conducted as part of the refurbishment.

The boom dynamics testing was carried out in the following manner: Since the presence of gravity creates nonlinearities in long flexible systems, a two-phase approach was carried out to characterize the deployed boom. In the first phase, the booms were deployed down and loaded transversely, from which load-deflection curves were measured. Tests were performed in the two transverse directions (tab plane and slot plane) and for two different lengths. Figure 16 shows one of these tests underway. In a sequence of nonlinear analyses (including the stiffening effect of gravity), the root stiffness and boom EI were determined. In the second phase, the booms were again deployed down, and a transverse step deflection was applied in each of the two transverse directions, then released. Here, the first natural vibration frequency for each direction, using the time histories of the boom deflections, was measured and compared to the measured first natural frequency, from tests of the booms in the horizontal (water table) configuration. The natural frequency of the booms with a $305 \mathrm{~g}$ 
Fig. 16 Boom element testing in vertical direction

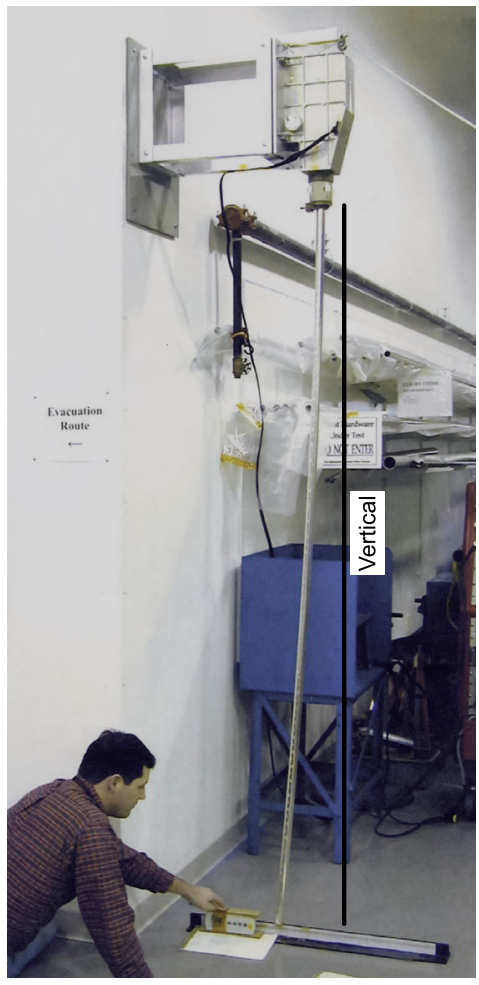

tip mass was determined to be $0.113-0.134 \mathrm{~Hz}$. The flexural rigidity (EI) of the boom elements was measured to be $122 \mathrm{Nm}^{2}$ and $83 \mathrm{Nm}^{2}$ for the slot and tab planes, respectively. The boom element linear density was $110 \mathrm{~g} / \mathrm{m}$. The mass of the spherical sensor (including shadow equalizer and pre-amplifer) was $305 \mathrm{~g}$.

The booms underwent spacecraft environmental testing per C/NOFS specifications. Test and alignment procedures were developed to meet C/NOFS VEFI requirements. Acceptance testing included: (1) functional testing, (2) vibration, (3) thermal vacuum, including partial deployment at temperature extremes, (4) post T/V functional testing, and (5) boom alignment or straightness measurements deployed on a water table.

\subsubsection{Boom Thermal Considerations}

The booms consisted of $2.86 \mathrm{~cm}$ diameter $\mathrm{BeCu}$ tubes perforated to provide better equalization of solar illumination-induced heating on both sides of the boom element. The heat conducted through the tube and inter-locking tabs, as well as radiative transfer in the blackenedboom interior kept the outer side of the boom (away from the sun) at the same temperature as the inner side, minimizing thermal deflection. The outside element was coated with polished silver to reflect the majority of the solar irradiance, and minimize the heat input to the boom, and then coated with Teflon, as had been carried out for the DE-2 electric field experiment (Maynard et al. 1981). The Teflon protected the silver from oxidation and provided electrical insulation of the outside of the boom. Thermal analysis of the modified C/NOFS booms with the internal ribbon cable was conducted to determine the alignment limits of the 

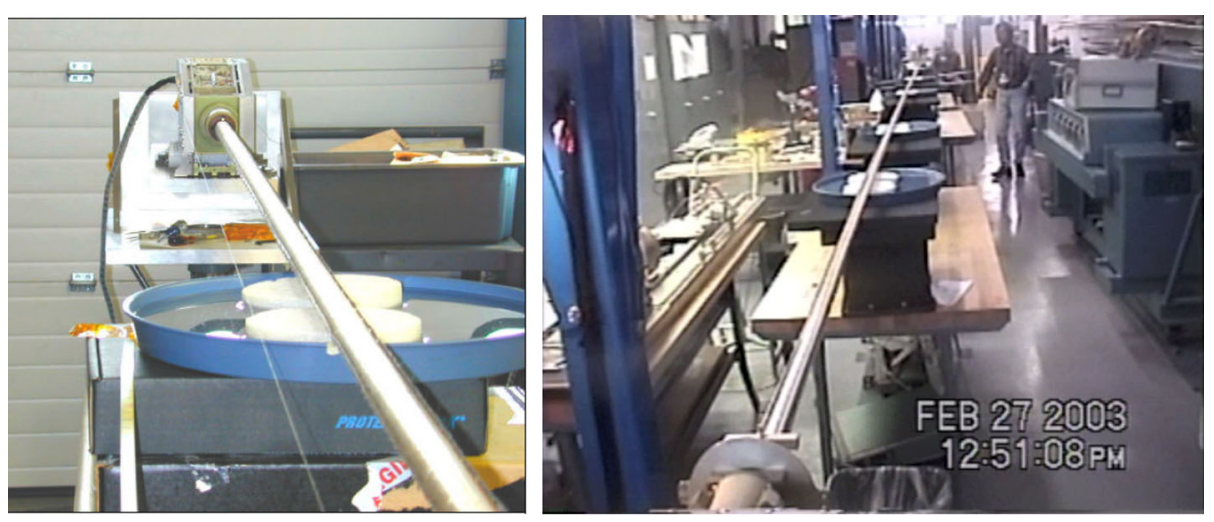

Fig. 17 Boom element testing on water table in horizontal direction

deployed boom and to verify that the cable's slight blockage of the hole perforation did not compromise the thermal response integrity of the booms.

\subsubsection{Inner Sensors}

At a distance of $2 \mathrm{~m}$ in-board of the outer spheres, the outer element Teflon was removed for a length of $25 \mathrm{~cm}$ to enable the boom element to come in contact with the plasma and serve as an independent electric field sensor. This sensor thus behaved in a manner similar to that of the DE-2 sensors, although in the case of DE-2, the outer, exposed, sensor was $2 \mathrm{~m}$ long and was at the end of the boom. The inner sensors were included to provide redundant sensors for the spheres, since the supply and signal wires for the spheres in the center of the booms constituted a new design. They were also included to enhance the accuracy of the DC electric field observations by providing an independent measurement along the same axis, as well as providing for plasma wave interferometry with multiple baseline measurements along a given axis. The cylinder sensors had separate pre-amps situated at the bases of the booms for both DC/VLF and HF wave electric fields, as on DE-2. This enabled comparisons of the HF wave characteristics measured with two sensor types. The cylindrical boom elements were directly connected to the input of a DC/VLF high impedance pre-amp, as well as through a capacitor to HF pre-amps.

\subsubsection{Boom Installation and Straightness and Geometry Considerations}

The VEFI boom requirements specified that the boom element shall be sufficiently straight such that the vector defined by the center of the sphere to the base of the boom element shall not deviate by more than 1.0 degree (requirement). The actual performance (shown below) was closer to 0.3 degree (3-sigma) from the nominal center line fiducial during operations.

Boom straightness was determined prior to launch on water-tables in which the booms were fully deployed, and their straightness measured (see Fig. 17). Slight, repeatable bending of 1-2 degree per boom was observed and carefully measured for each boom on the water table as well as during vertical alignment testing in which the fully deployed element was hung from above. Such inherent, repeatable bending was compensated for with shims when the booms were installed in the spacecraft (see Fig. 13).

The two booms along the direction perpendicular to the orbit plane (booms 1 and 2) were each situated "back-to-back" and along the center line. Space limitations within the 
Fig. 18 Diagram showing deployer positions of the 4 booms in the orbit plane canted to optimize orthogonal orientation after full deployment

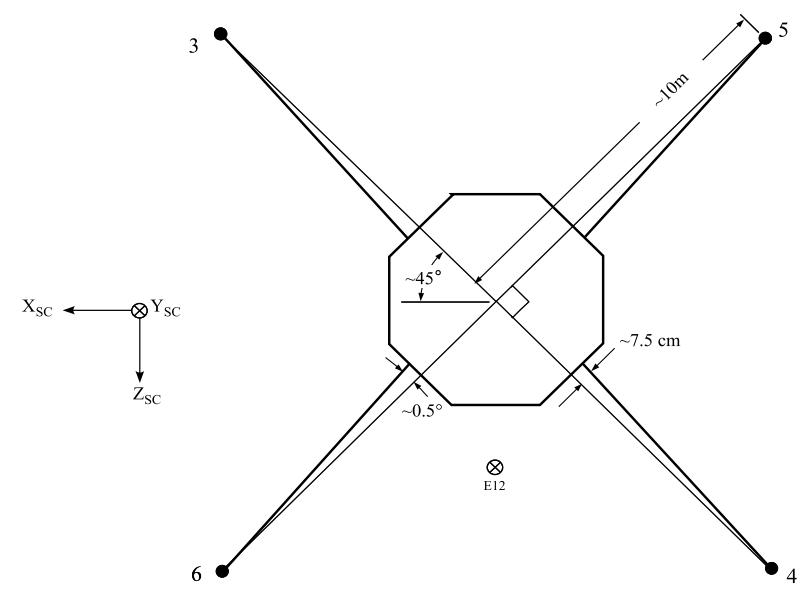

spacecraft precluded the installation of opposing electric field booms "back-to-back" in the perpendicular orbit plane. Thus, the booms situated in the orbit plane (the " $3-4$ " and "5-6" booms) were installed such that opposing booms were alongside each other and offset from the center of the spacecraft. As shown (not to scale) in Fig. 18, these booms were slightly canted (by 0.5 degrees) so that, when fully deployed, the 6 spheres formed an orthogonal set of 3-axis measurements. Alignment mirrors on each boom (see Fig. 13) enabled the specific orientation of the each deployer to be ascertained, from which a non-orthogonality matrix was calculated.

Furthermore, once in orbit, an updated non-orthogonality matrix of the booms was determined based on comparisons of the measurements with $\mathbf{V} \times \mathbf{B}$ and other data. The initial non-orthogonality matrix could then be used to apply very small corrections to the data. Adjustments to the data by the on-orbit non-orthogonality matrix accounted for corrections of less than $1 \mathrm{mV} / \mathrm{m}$. As shown on orbit, thermal variations of the boom straightness were small. Using analysis of $\mathbf{E} \cdot \mathbf{B}=0$ and comparisons of the measured signals to $\mathbf{V} \times \mathbf{B}(\sim 200 \mathrm{mV} / \mathrm{m})$ in regions where the ambient electric field was $<1 \mathrm{mV} / \mathrm{m}$, the boom straightness was shown to deviate by less than 1 degree, as discussed further on below.

\subsubsection{Spheres and Coating}

Spherical electric field sensors were chosen to optimize performance and achieve the required accuracy. Because of the large boom diameter $(2.86 \mathrm{~cm})$, the sphere diameter was chosen as $11.8 \mathrm{~cm}$. This also minimized the shadow asymmetries caused by the shadow of the boom during daytime when the sun angle to the boom was large (see below). The large sphere diameter also provided a significant input capacitance $(\sim 6.6 \mathrm{pF})$ to enable high quality wave measurements including the HF regime.

In order to achieve a uniform, non-varying work function, the aluminum spheres were coated with several layers of carbon using a graphite colloidal (DAG213) compound. The carbon was "baked on" in the GSFC thermal coating facility. This process had been carried out by the Goddard team for dozens of electric field sensors flown on sounding rockets as well as for the DEMETER electric field sensors (Berthelier et al. 2006).

The carbon also provides an absorption/emissivity (alpha/epsilon) thermal property ratio of 0.89 , which ensured a benign thermal environment of the inside of the spheres where the preamps were located. The temperatures of the sphere interiors were monitored throughout 
the mission. The average temperatures over the course of the mission ranged from -15 to $20 \operatorname{deg} \mathrm{C}$ over the course of the 7.5 year mission, as shown in the Appendix.

The team considered the effects of atomic oxygen erosion of the carbon surface. To ameliorate these concerns, adequate coatings were included that resulted in an approximate thickness of 3 mils ( $\sim 76$ microns). No substantial changes in the work function response were noted during the mission. Slightly higher temperatures were observed later in the mission (after 6 years of orbit) when perigee had declined, as shown in the Appendix. This may have been due to the higher density of the upper atmosphere at the lower altitudes as well as possible changes in the coating.

\subsubsection{Spherical Sensor Harness (Ribbon Cable Inside Boom)}

The signals to and from the sphere pre-amp were transmitted via a small, miniaturized ribbon cable $(7.6 \mathrm{~mm}$ in width with a thickness of $0.28 \mathrm{~mm}$ ) with 8 copper conductors of 0.005 inch diameter each that was placed in the center of the cylindrical elements and rolled up with the boom. The wire/ribbon was manufactured by Tayco Corp. (California, USA) and was selected for its durability to withstand repeated deployments/retractions during testing on the ground and possibly in orbit, as well as its thermal properties. A segment is shown in Fig. 14. The ribbon was made of kapton and connected to Omnetics 12-pin circular connectors within the sphere and also with the base of each deployer unit.

The Kapton ribbon material was yellow/orange color. The Kapton ribbon was selected for its ability to withstand UV radiation and its durability. The VEFI team concluded that Teflon film was too thin and susceptible to breaking, and that copper foil might not withstand pressing and pulling. Thermal analysis showed that the blockage of the holes by the Kapton ribbon would be minimal, and indeed the boom straightness was maintained throughout the mission (see discussion further on below).

\subsubsection{Shadow Equalizers}

When exposed to solar ultraviolet radiation, conductors emit photoelectrons and create a current in conductors. Specifically, this occurs for electric field sensors exposed to sunlight. When exposed to uniform illumination, matching spheres with identical and uniform work functions, emit the same number of photoelectrons, and their potential difference is not affected. If the illuminated areas are unequal, however, due to different degrees of shadowing, a potential difference appears, which is interpreted as an electric field. This situation is shown in Fig. 19. If the sun's direction was perpendicular to the double probe, the two spheres would receive equal fluxes of photons directly from the sun and their current contributions would be balanced. (We ignore secondary photoelectrons from the spacecraft which are negligible for double probe measurements conducted within the ionosphere using booms long relative to the spacecraft dimension.) However, if the sun's rays were not orthogonal to the double probe and particularly if they were aligned closely with the double probe direction, the sphere furthest from the sun would be shadowed by its boom, resulting in an imbalance of the photons collected by the two probes, as shown in the upper panel of Fig. 19.

There are two standard means to mitigate these effects. First, the ratio of the sphere diameter to the boom diameter should be as large as practical. A second approach is to simply extend a rod of the same diameter as the central boom outward from the spheres to produce equal shadow effects on each sensor for all solar illumination angles. As shown in the lower illustration of Fig. 19, such a rod produces equivalent shadows on the opposing spheres to create effectively balanced photoelectron currents. 

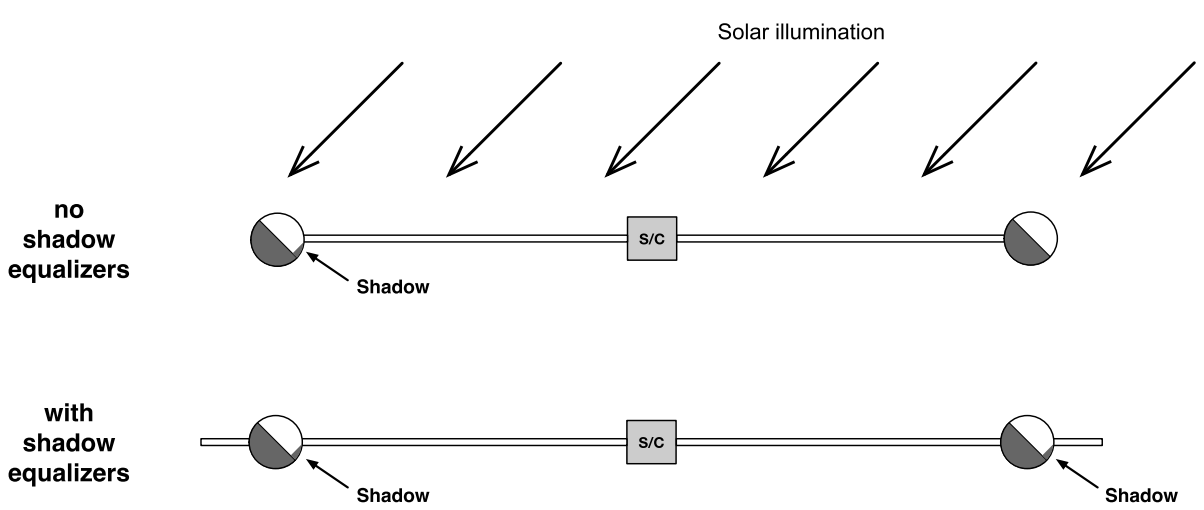

Fig. 19 Illustration of the solar photoelectron imbalance due to boom shadowing of a single sphere (upper diagram) that is alleviated with balanced shadowing of both spheres when boom segments are included outboard of the spheres (lower diagram)

Fig. 20 Cumulative distribution function of boom shadow mismatches for different length shadow guards or "equalizers"

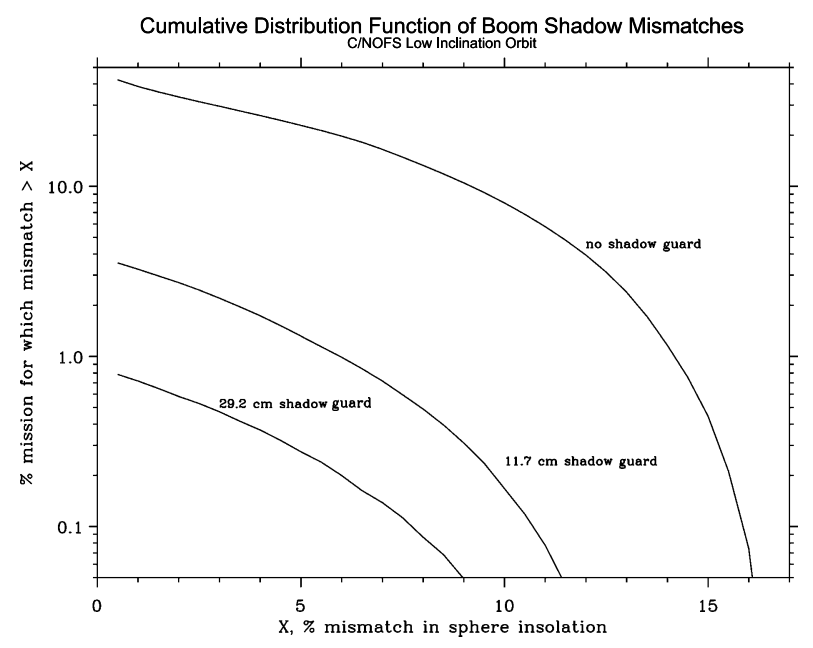

Figure 20 shows calculations of the expected imbalance of photoelectrons when no shadow equalizer is used as well as how this imbalance is mitigated by the inclusion of shadow equalizers of different lengths. The calculations have been averaged over all sun angles encountered by the VEFI probes during the daytime for a two-year period. Such calculations vary with the spacecraft inclination and the effects are most pronounced for a low inclination satellite with double probes in the orbit plane, as is the case for the VEFI instrument on C/NOFS. Note that a shadow equalizer of one sphere diameter would greatly reduce this photoelectron imbalance, with improved optimization (smaller photoelectron imbalance) with still longer rods.

The asymmetric photoelectron effects are most pronounced for low inclination satellites when the sun angle is nearly aligned with the boom direction. For C/NOFS, this occurs at sunrise and sunset for the 3-4 and 5-6 booms in the orbit plane, as shown in Fig. 21. Because measuring accurate electric fields during the sunset local times was of particular importance for the C/NOFS objectives, the VEFI electric field booms each included a sufficiently long 
Fig. 21 Diagram showing how spheres on the booms in the orbit plane are susceptible to shadow mismatches, particularly near sunset and sunrise
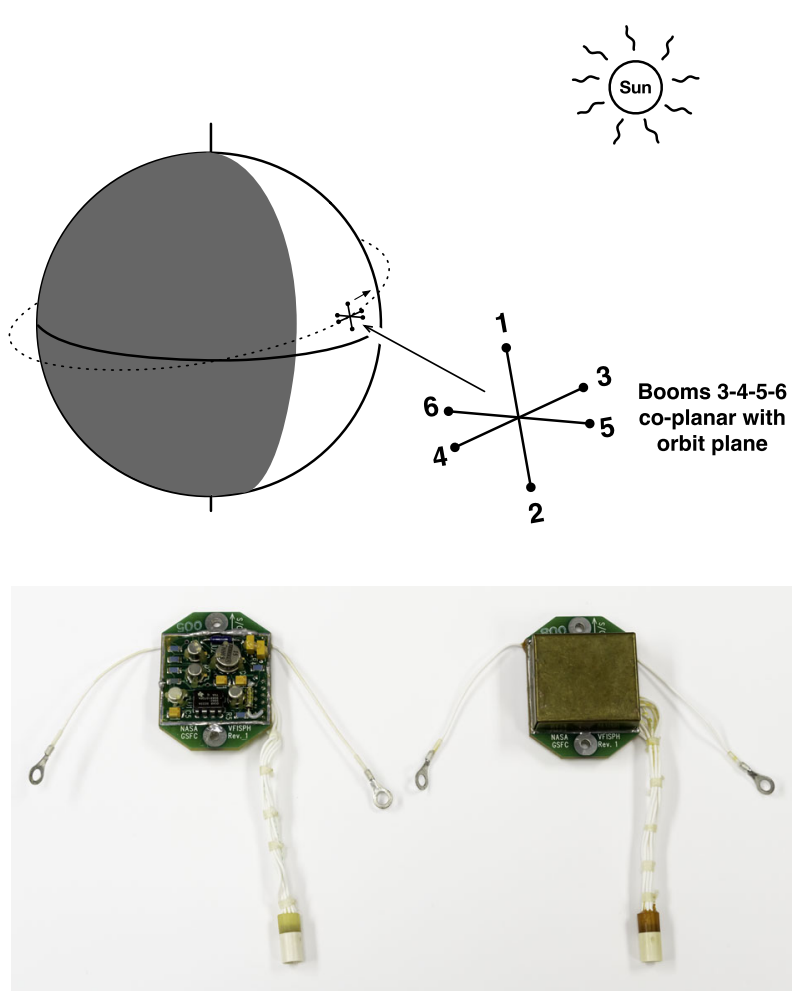

Fig. 22 Photograph of the sphere pre-amp circuit board with temperature sensor. The left-hand photograph shows the circuit without the guard. The right-hand photograph shows the circuit with the guard

shadow equalizer (approximately 2.5 times the sphere diameter) to minimize the effects of photoelectron imbalance when the double probes were closely aligned with the sun's direction. Although this effect would not be pronounced for the "1-2" boom which was essentially perpendicular to the sun at all local times, the shadow equalizers were included on all of the VEFI spheres to afford uniformity of all of the sensors.

\subsection{Electric Field Pre-amps in Spherical Sensors and Bias Circuit}

The spherical sensor pre-amps included a high input impedance $\left(>10^{12} \mathrm{ohm}\right)$ device (OPA128) with circuity at the input to provide protection against electrostatic discharge during ground handling. The pre-amps drew a total power of $\sim 40 \mathrm{~mW}$ (including that needed for the thermal sensor) and were encased in thin brass enclosures that were driven at the output of the pre-amp to serve as a guard. A photograph of the sphere preamp electronics with and without the guard is shown in Fig. 22.

The main electronics included circuitry to provide a programmable positive bias current to the spherical sensors from 0 to $57.25 \mathrm{nA}$; this was selectable from the ground. A temperature sensor was situated on the pre-amp electronics board in each sphere.

\subsection{Pre-amps for Inner "Cylinder" Sensors}

The pre-amps for the inner cylinder sensor were located at the base of each boom. Figure 23 shows a cylinder preamp inside its EMI controlled box, with the cover removed. As with the Dynamics Explorer-2 electric field experiment (Maynard et al. 1981), these pre-amps 
Fig. 23 Photograph of the cylinder pre-amp box with its circuit board visible which was at the base of the booms that accepted the cylindrical sensor input

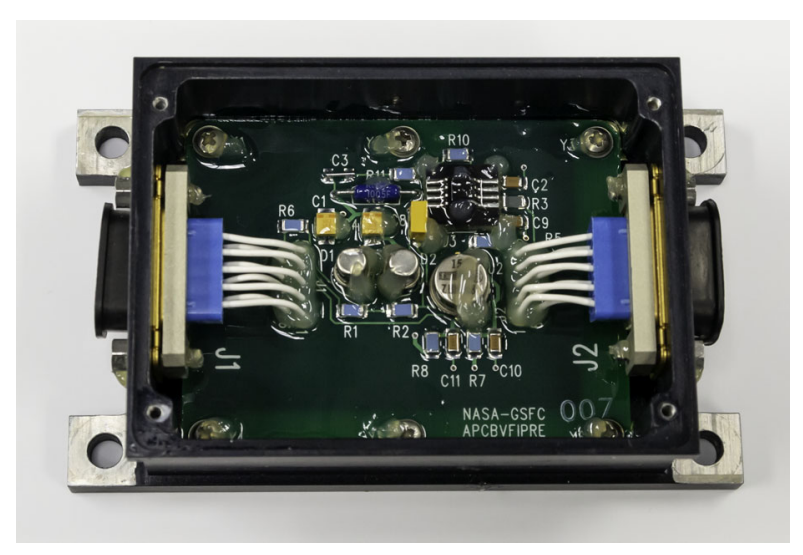

were driven directly by the cylinder conducting element from which the signal was divided to provide dual inputs: DC/VLF data via the OPA128 operational amplifier and HF data via the TLE2037A operational amplifier. The latter took advantage of the long cylinder antenna to provide higher fidelity data at HF. The overall design of the cylinder pre-amps included anti-ESD devices at their input, in a similar fashion to that of the sphere pre-amps.

\subsection{Overview of the Main VEFI Electronics, Electronics Box, and Power Supply}

Overview The main electric field analog electronics ensemble consists of differential amplifiers, analog low pass and bandpass filters, bias and Langmuir probe circuitry, and 16-bit A/D converters. The digital electronics consists of an IDPU, a DSP processor, and an 8 Mbyte memory including burst capabilities to capture triggered "snapshot" data collection. The electronics represent an evolved heritage based on past Goddard electric field instruments on the low earth-orbiting satellites such as Dynamics Explorer-2 and San Marco, launched in 1981 and 1988, as well as DC/AC electric field experiments flown on over 50 sounding rocket missions over 30 years, which included digital signal processing (FFT algorithms including spectral matrices) and burst memories.

A diagram providing an overview of the main VEFI electronics and signal flow is shown in Fig. 24. The diagram shows the electric field pre-amps, diff-amps, DC and AC electronics included low pass, boosted, and bandbass filters, a filter bank, and the on-board signal processing (FFTs) and burst memory with associated digital filters. Input signals from the magnetometer, fixed-bias Langmuir probe, and lightning detector are also shown. Their electronics are discussed in subsequent sections below.

Note that this diagram (Fig. 24) is provided as a symbolic rendering of the VEFI electronics since the actual diagram would be too cumbersome to reproduce in this article. Here, we show the different input signals to the VEFI electronics box and the different processing functions within the box. Not all signals on the left can be routed to all of the boxes on the right.

Before discussing the analog and digital circuits that comprised the electric field experiment, we describe the main electronics box itself.

\subsubsection{Main Electronics Box}

The VEFI experiment consists of analog and digital circuitry divided among different electronics boards housed in trays that were stacked as shown in Fig. 25. Each modular electronics tray has a discrete function and includes one or two boards. Rather than have a back 


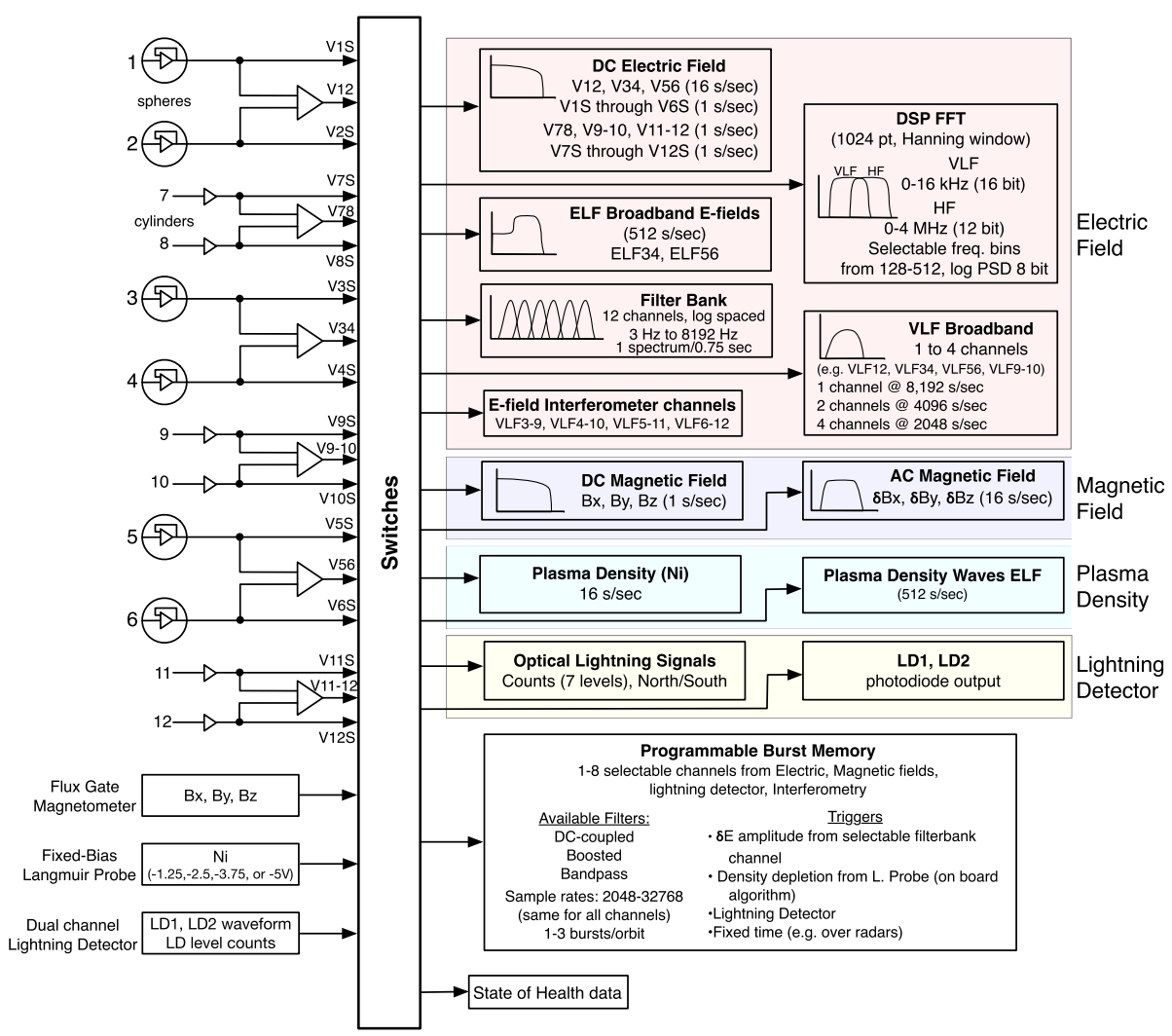

Fig. 24 Block diagram of the VEFI sensor inputs (left) and different signal processing functions (right) for the electric field, magnetic field, plasma density, and lightning detector. The programmable burst memory, which accepted inputs from all of the sensors, is also shown

plane, connections between the trays were made with external "tray-to-tray" harnesses. The trays received input from the appropriate sensors and transferred signals/data between trays and telemetry, as appropriate. This facilitated testing of individual boards and maintained a tight EMI control of each tray. The entire box was then encased with lightweight panels to further ensure EMI control and meet thermal requirements; the outer panels were coated with black anodized paint, as shown in Fig. 26. Essentially, the main VEFI electronics consisted of a "box within a box" to minimize EMI and modularize elements and facilitate testing. The telemetry connections to the spacecraft were via a MIL-STD-1553B interface and connector, the Trompeter BJ3157.

\subsubsection{Power Supply and Converter}

The VEFI power supply consisted of a highly efficient, low noise regulator board that was designed and built at Goddard and which had flown on numerous satellite instruments. Power from the spacecraft consisted of $+28 \mathrm{~V}( \pm 6 \mathrm{~V})$ which was regulated on the VEFI power board, which was situated at the base of the box to efficiently dissipate its heat through the bottom deck. The main power was regulated with a DC/DC converter operating at $50 \mathrm{kHz}$ that was $\sim 80 \%$ efficient. Secondary, regulated voltages of $+12 \mathrm{~V},-12 \mathrm{~V}$, 

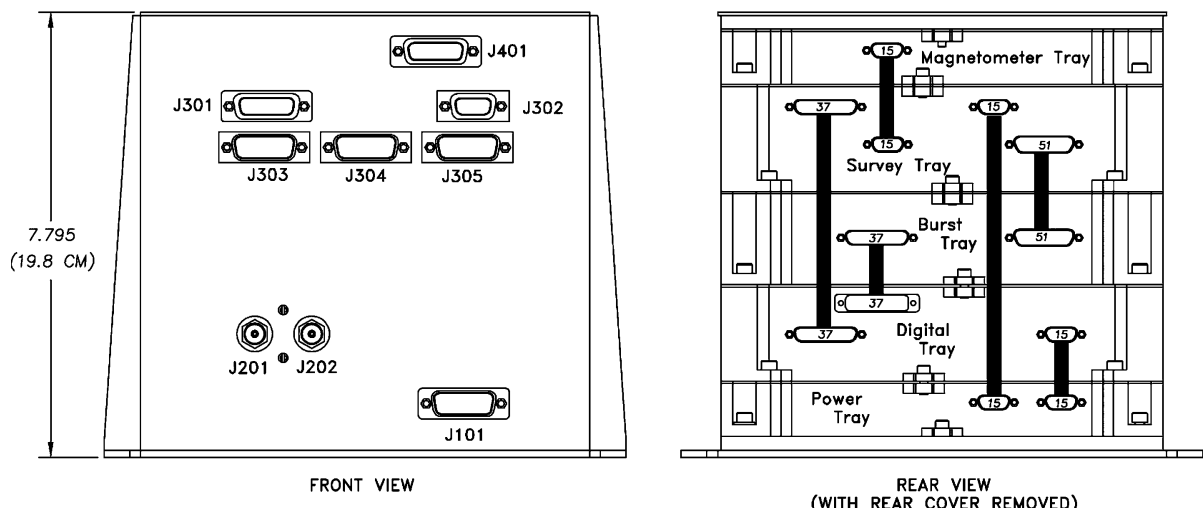

Fig. 25 Drawing of main electronics box showing connectors and inter-tray harnesses for the front (left) and rear (right) sides with the outer cover removed
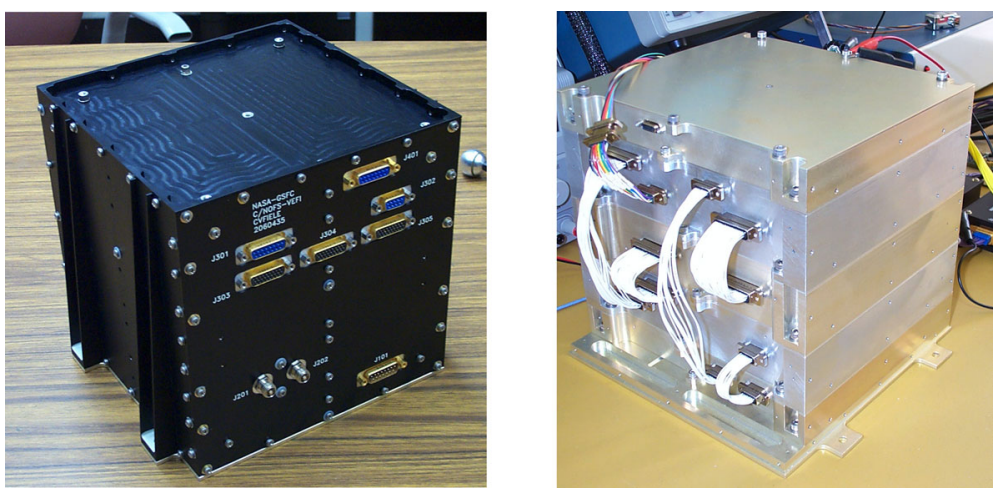

Fig. 26 Photographs of the main electronics box with the outer cover attached (left) showing the connectors to the various sensors and the spacecraft power and telemetry interfaces and without the outer cover (right) showing the inter-tray harness connections

and $+5 \mathrm{~V}$ were provided to the various components of the VEFI experiment, including the pre-amps. A greater than $1 \mathrm{M}-\mathrm{Ohm}$ isolation existed between primary and secondary power systems.

The actual VEFI power was mode dependent. For the full measurement complement (all sensors), the power was 11.8 Watts. A low power mode without the DSP or burst memory was 8.7 Watts.

\subsection{Electric Field Analog Channels}

The electric field analog channels consisted of differential amplifiers, DC-coupled data with low pass filters, quasi-DC or "boosted" DC/ELF data, bandpass VLF, and bandpass HF data. The different channels and associated filter characteristics are discussed below.

DC Electric Fields Including Direct Pre-amp Potential Measurements Vector DC electric fields are gathered from potential differences between opposing sensors as discussed above. The difference amplifiers provided difference voltages between the pre-amp output of the 
sensors, using matched resistors with excellent common mode rejection. The pre-amp output voltage is referenced to the spacecraft common or "skin" voltage. Designating this signal as $\mathrm{VaS}$, where $\mathrm{a}$ is the sensor number and $\mathrm{S}$ is the spacecraft skin voltage, we use the nomenclature Vab where $\mathrm{Vab}=\mathrm{VaS}-\mathrm{VbS}$ to define the electric field differential channels. Thus, the experiment included the following double probe potential difference measurements: V12, V34, V56 for the sphere pre-amps and V78, V9-10, and V11-12 for the cylinder pre-amps (see Fig. 8 for the sensor positions).

All DC electric field "survey" channels are sampled at $1016.26521 \mathrm{~s} / \mathrm{sec}$ by three $16.260243 \mathrm{kHz}, 16$-bit A/D converters preceded by analog multiplexers. These signals are downlinked at much slower rates, and the decimation takes place through a digital averaging process. The initial design called for A/D converters sampling at $16.384 \mathrm{kHz}$. However, to avoid beating with the polling clock of the spacecraft 1553 interface which could vary depending on temperature swings, the VEFI sampling rate for these channels was slightly reduced to provide an optimum interface and guarantee there would be no buffer overflow. Although we frequently refer in this article to DC and ELF data sampled at $1 \mathrm{~s} / \mathrm{sec}, 16 \mathrm{~s} / \mathrm{sec}$, and $512 \mathrm{~s} / \mathrm{sec}$, the precise sample rates were slightly lower, corresponding to $1.0 \mathrm{~s} / \mathrm{sec}$, $15.9 \mathrm{~s} / \mathrm{sec}$, and $508.1 \mathrm{~s} / \mathrm{sec}$, respectively.

The main DC electric field channels included a fourth order low pass filter with a $3 \mathrm{~dB}$ point at $7 / 8$ of the Nyquist frequency. The anti-aliasing filter cut-off frequencies generally reflects the downlink sample rate, not the A/D sampling rate. The data for each spherical double probe channel was produced at $16 \mathrm{~s} / \mathrm{sec}$ and at $1 \mathrm{~s} / \mathrm{sec}$ for the cylinder double probes. Each channel had unity gain. The range of each spherical double probe component was $\pm 450 \mathrm{mV} / \mathrm{m}$ and had the frequency response shown in Fig. 27a.

In addition, each preamp output (V1S through V12S) was also digitized and telemetered to the ground at $1 \mathrm{~s} / \mathrm{sec}$ for each sphere and cylinder using 16-bit A/D converters. The average of two opposing pre-amp voltages provides a measurement that depends on the spacecraft potential and, in low plasma density environments, can provide information on the ambient plasma density. Furthermore, the individual sphere voltages can be combined to form other double probe voltage potential different pairs, such as V23, V24, V45, and V46. Although less accurate due to the effects of asymmetry, such measurements from different geometries provide a good check on the final vector electric field measurements, as well as providing redundancy. High-time-resolution measurements from the separate pre-amp outputs also provide "spaced receiver" measurements, providing interferometric measurements of wavelength and phase velocity of certain plasma modes.

AC or Wave Electric Fields The VEFI instrument also included a number of AC or wave electric field measurements. These were gathered in a number of different ways, depending on their frequency and desired time resolution:

1. Broadband wave data including "boosted DC/ELF channels"

2. On-board power spectral densities in the VLF and HF frequency domains

3. Power data from a "filter bank" composed of 12 individual channels from $3-8000 \mathrm{~Hz}$

4. Burst memory capture of 1-8 channels sampled at rates up to $32.768 \mathrm{ks} / \mathrm{sec}$ per channel and triggered on a variety of input criteria (described below).

Due to telemetry limitations, the AC broadband measurements in the ELF and higher frequency regimes were not continuously available. To optimize the use of the available telemetry, during normal operations, the broadband DC/ELF data and burst memory operated on the nightside, with the burst memory data stored in memory and transferred to the spacecraft during the dayside portion of the orbit. The different modes consisting of VEFI slow survey 
(a)
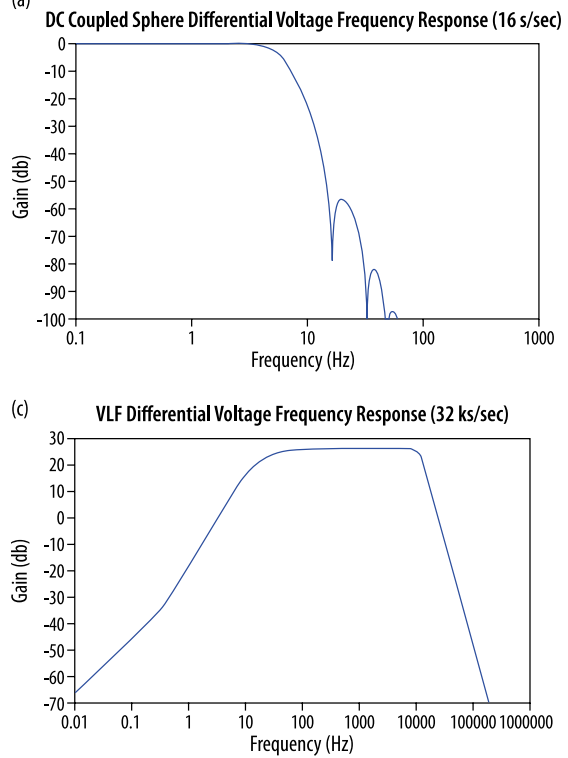

(b)

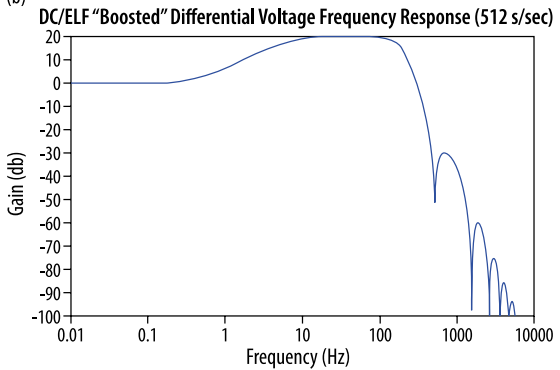

(d)

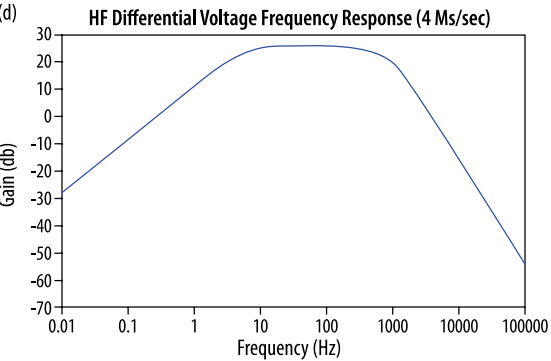

Fig. 27 Transfer functions (showing signal amplitude power) of the various VEFI filter types, including: (a) DC coupled data, (b) DC-coupled with enhanced or "boosted" ELF data, (c) VLF differential bandpass filter, and (d) HF differential bandpass filter

and fast survey data are described further on below. The filter bank data and on-board power spectral density data were gathered continuously at all local times.

Broadband DC/ELF Data Broadband DC/ELF data were gathered from 2 channels continuously at $512 \mathrm{~s} / \mathrm{sec}$ in the nominal mode. The input for the $512 \mathrm{~s} / \mathrm{sec}$ broadband data were from either two selectable electric field components (ELF34 or ELF56) or one selectable electric field component and the output of the VEFI fixed-bias Langmuir probe (discussed below). Each electric field signal had a 4-pole low pass filter with a $3 \mathrm{~dB}$ point at 7/8 of the Nyquist frequency, as shown in Fig. 27b. The ELF electric field broadband components were DC coupled with a boosted, 1-pole high pass filter at $5 \mathrm{~Hz}$ above which the signal was amplified by a mid-band gain of 10 .

Broadband VLF Data (High Rate Mode) The VLF broadband data were gathered from 1, 2, or 4 selectable electric field channels that included these differential pairs: VLF12, VLF34, VLF56, and VLF9-10. The data were available at $2048 \mathrm{~s} / \mathrm{sec}$ (4 channels), $4096 \mathrm{~s} / \mathrm{sec}$ ( 2 channels), or $8192 \mathrm{~s} / \mathrm{sec}$ ( 1 channel) when the C/NOFS telemetry was in the fast mode. The high pass filters in each case were fixed at $10 \mathrm{~Hz}$. The low pass filters rolled off $(-3 \mathrm{~dB}$ points) at frequencies at $7 / 8$ Nyquist frequency that were selectable via a digital filter. The channels included a gain of 20 .

Power Spectral Density VEFI included on-board FFT computations in both the VLF and HF modes for any selectable electric field channels. The FFTs consisted of 1024-point FFTs with Hanning windows from which power spectra densities were computed on board from 
Fig. 28 VEFI filter bank. (a) block diagram of main filter bank architecture, (b) Photograph of miniaturized filter bank circuitry, (c) 12

logarithmically-spaced filter bank frequencies

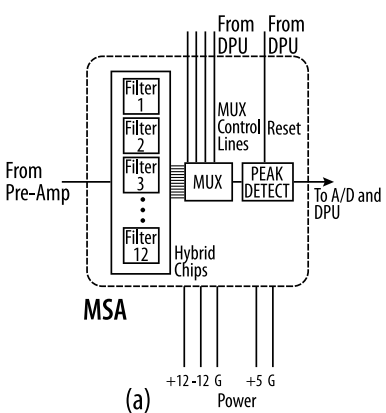

(a)

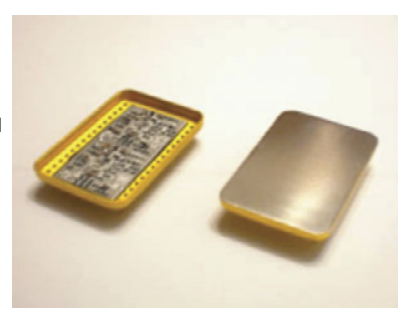

(b)

Filter Bank Channels

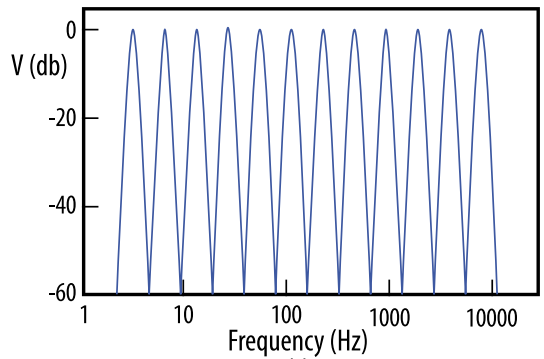

(c)

the sum of the squares of the real and imaginary components and ensemble averaging of the data were performed. The amplitudes were logarithmically compressed and telemetered using 8 bit words. Because of telemetry limitations, 128 logarithmically spaced frequencies were selected from the available 512 frequencies for continuous transmission to the ground at a nominal rate for both the VLF and HF spectra of every 2 seconds. At certain times, however, all 512 frequency bins were transmitted to the ground and/or the spectra were transmitted at a higher rate, up to $64 \mathrm{spectra} / \mathrm{sec}$ depending on the available telemetry.

The VLF spectra are computed from potential differences from any selectable electric field double probe with a 4-pole low pass Butterworth filter at $12.2 \mathrm{kHz}$, a low pass filter at $10 \mathrm{~Hz}$, and a mid-band gain of 20, as shown in Fig. 27c.

VEFI included HF transfer functions with different, selectable low pass filters, for example at $1 \mathrm{MHz}$ with a gain of 18 or at $1.5 \mathrm{MHz}$ or $3 \mathrm{MHz}$, each with a gain of 36 . The low pass filter was determined by the selected sample rate, which was nominally $8 \mathrm{Ms} / \mathrm{s}$, but could be $4 \mathrm{Ms} / \mathrm{s}$ or $2 \mathrm{Ms} / \mathrm{s}$. Lower sampling rates, for example at $500 \mathrm{ks} / \mathrm{s}$, could also be chosen to emphasize lower frequencies. In these cases, a digital filter was employed as an anti-aliasing filter.

Filter Bank Data VEFI included a multi-channel spectrum analyzer (MSA) or "filter bank", as shown in Fig. 28, to gather continuous spectral data of electric field signals in logarithmically spaced frequency bands from 3-8000 Hz. The output of each filter bank channel corresponded to the power within the specified band and was digitized with 16-bit words. The MSA consisted of 12 analog channels forming a "filter bank" that was telemetered at 1 spectra every 0.75 seconds (or $1.33 \mathrm{spectra} / \mathrm{sec}$ ). The multi-channel spectral analyzer frequencies were selected to examine ELF and VLF wave activity associated with Spread-F and other local irregularities as well as ambient plasma waves. Importantly, selected signals from the MSA were used to trigger the burst memory that gather snapshots of high-rate 
time series data at interesting times in the mission. The filter bank data served to provide evidence of irregularities in low telemetry rate (forecast mode) data for the C/NOFS project via TDRSS.

The 12 analog channels forming the filter bank utilized miniaturized hybrid circuitry, as shown in Fig. 28. The design was based on previous GSFC design work for a planetary experiment. Each hybrid included two channels, with 6 hybrids total. The hybrid electronics fabrication was sub-contracted to Quad Tron, Inc., in Pennsylvania (USA).

The ensemble mass of the MSA analog electronics was $300 \mathrm{~g}$ and utilized power of $0.5 \mathrm{~W}$, which was part of the main VEFI electronics budget. The 12 analog channels which formed the filter bank included log-spaced frequency coverage from 3-8000 Hz, with a channel Bandwidth of $20 \%$, as shown in Fig. 28. The channels included 6th-order linear phase filters and were designed to minimize filter ringing. The signals from each filter were log-compressed with $>90 \mathrm{~dB}$ of dynamic range and were represented by 8 bit words. Channel selectivity was better than $-55 \mathrm{~dB}$.

The MSA block diagram is shown in Fig. 28. The input to MSA consisted of one bipolar analog line from an electric field differential amplifier. Electric field data could be selected from 4 MUX control lines and included a detector reset. It was powered with $\pm 12 \mathrm{~V},+5 \mathrm{~V}$. The output from MSA consisted of a unipolar analog output between 0-5 V. The output was digitized at $85.333 \mathrm{~s} / \mathrm{sec}$ and further averaged in the IDPU. The analog MUX switched between channels at $0.97 \mathrm{msec}$ with a single spectral sweep lasting $11.6 \mathrm{msec}$. Below $40 \mathrm{~Hz}$, the channels were internally over-sampled. The IDPU averages values to the slower return rate of 1,8 , and 64 sweeps per second. The nominal rate was 0.75 seconds for a full 12 frequencies.

\subsection{Analog/Digital Converters and MUX Circuits}

The VEFI digital electronics gathered data from all the instrument sensors (DC and AC electric fields from spheres and cylinders, magnetometer, Langmuir probe). It digitized the data, averaged the data, and in some cases, implemented digital low-pass filters (required for some wave channels sampled with variable rates). It also computed on-board calculations, identified burst data, and transferred data to the spacecraft telemetry system both real-time and from its internal memory.

VEFI's primary A/D converter was the 16-bit CS5016, which was run at a survey mode sample rate of $16.260 \mathrm{kHz}$ for DC and ELF data and at $32.768 \mathrm{kHz}$ for wave data intended for the VLF FFTs and the burst mode wave capture data (both DC-coupled and AC coupled data). The sample rate in burst mode could be selected between $2-32 \mathrm{kHz}$, as discussed further on below. The VEFI A/D converter for high frequency waves is the 12-bit SEi 9240LPRP converter, which ran at $8 \mathrm{MHz}$ sample rate.

The digital electronics controlled numerous MUX circuits to optimize the performance of the instrument and maintain flexibility for different signal inputs. Analog multiplexers were implemented to provide simultaneous sampling of critical signal triplets (e.g., Bx, By, and Bz; V12, V34, and V56). The MUX configuration provided redundancy for the core mission objectives in that critical signals could be routed to different A/D converters, on command, in the event of an anomaly.

In a later section, the digital architecture is described from the standpoint of the IDPU and DSP software and processors. We now present the other sensors that were part of the VEFI, the flux-gate magnetometer, fixed-bias Langmuir probe, and optical lightning detectors. 
Fig. 29 Rendering of the C/NOFS spacecraft showing the deployed magnetometer in the aft and the VEFI fixed-bias Langmuir probe directed towards the nadir

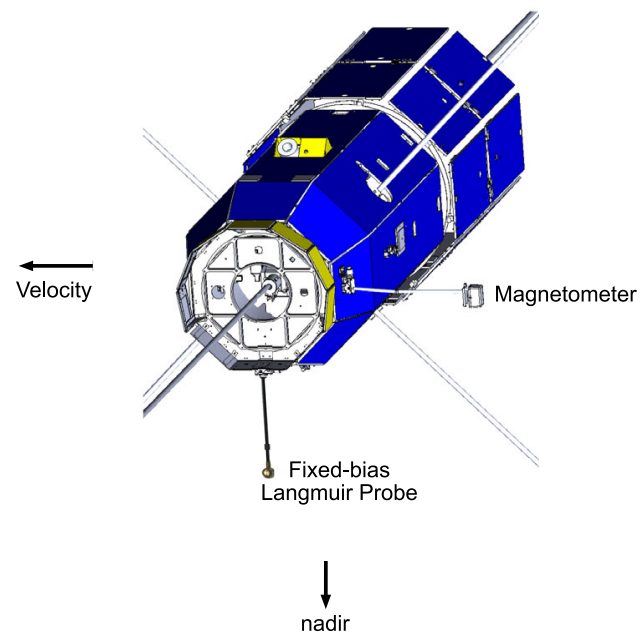

\section{Flux-Gate Magnetometer}

VEFI includes a flux-gate magnetometer configured to provide both DC and AC vector magnetic field measurements. The magnetometer measurements are used to compute and remove the $\mathbf{V} \times \mathbf{B}$ contribution to the measured electric field, to convert the measured electric fields to $\mathbf{E} \times \mathbf{B}$ velocities, to provide direct measurement of ionospheric currents, and to measure the magnetic field component of ionospheric disturbances and Alfven waves. Several additional scientific research topics were also addressed with the VEFI magnetic field data.

Vector DC magnetic field data are gathered with a flux-gate magnetometer with active thermal compensation extended on a $0.6 \mathrm{~m}$ boom, described below. Figure 7 shows a photograph of the VEFI magnetometer mounted on the $60 \mathrm{~cm}$ boom stowed against the spacecraft body. The orange/gold covering is the Kapton material included for thermal reasons. After deployment, the magnetometer was situated in the anti-ram direction of the spacecraft motion, as shown in Fig. 29.

Sensor Details The VEFI magnetometer was designed and built by Dr. Mario Acuna in NASA/Goddard's Laboratory for Extraterrestrial Physics and included a three-axis sensor at the end of a $60 \mathrm{~cm}$ boom, as described below, and a drive board that was part of the main VEFI electronics box, described above. The magnetometer is a tri-axial fluxgate magnetometer that uses the ring core technique (e.g., Acuña 1974). This flux-gate magnetometer has extensive heritage on a number of low earth orbit missions including the DMSP, Freja, and MAGSAT satellites. The same flux-gate design was also flown on a number of planetary missions, including Voyager, Mars Surveyor, and Mercury Messenger. A photograph of the flux-gate sensor showing orthogonal sensors is shown on the left-hand side of Fig. 30.

The magnetometer sensitivity was set to $\pm 45,000 \mathrm{nT}$ to account for the weaker magnetic field at the earth's equator. This still allowed for testing and calibration at mid latitudes at NASA/Goddard in Maryland (USA). The DC magnetic field data were sampled with a 16bit A/D converter at $1016.265 \mathrm{~s} / \mathrm{sec}$ for each component which was averaged to $0.992 \mathrm{~s} / \mathrm{sec}$ and included a low pass filter with a $3 \mathrm{db}$ point at $0.316 \mathrm{~Hz}$, as shown in Fig. 31. The LSB of the data was $1.4 \mathrm{nT}$. The floating-point averaged 24-bit word was telemetered with each sample, in order to slightly improve the sensitivity, adding the equivalent of 1-2 "extra" bits. 
Fig. 30 Photograph of the VEFI magnetometer showing the flux-gate coils on the left and the heater on the right
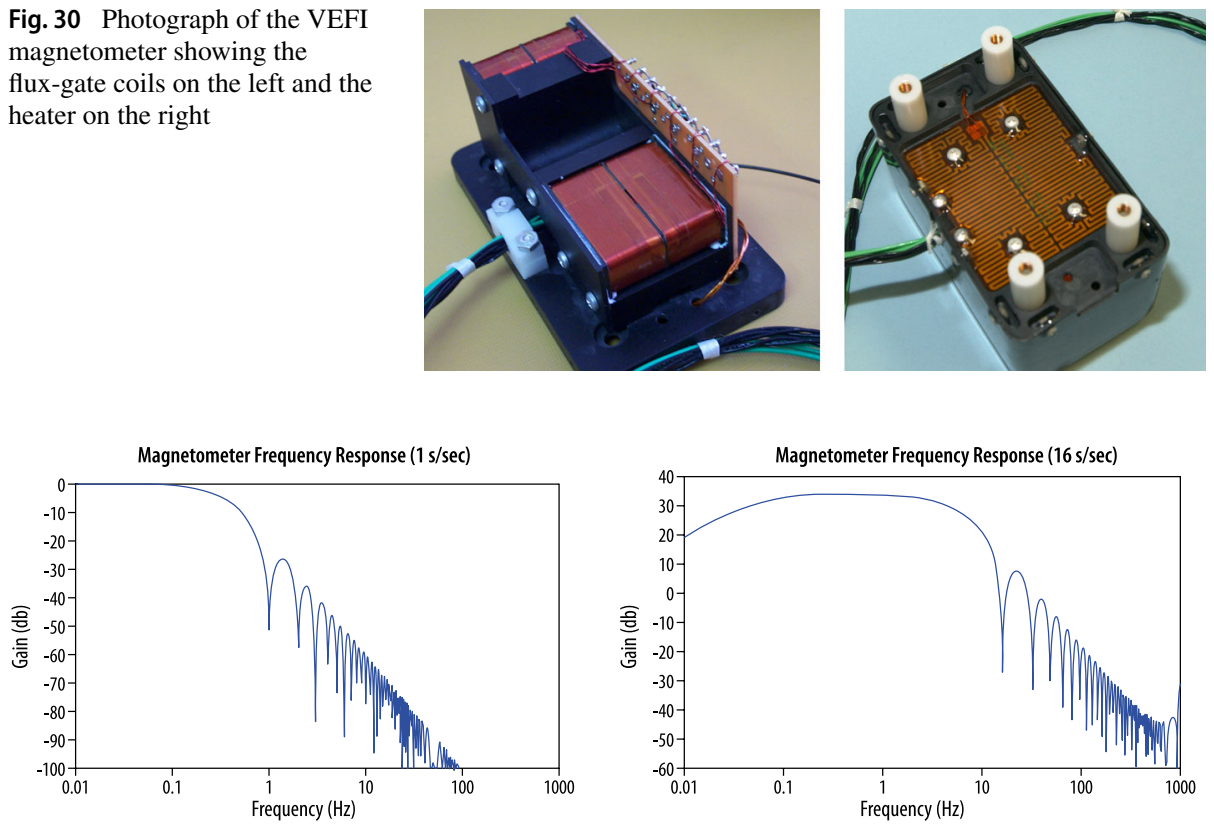

Fig. 31 Transfer functions of the DC-coupled (left) and AC-coupled (right) magnetometer channels

The three DC magnetometer components are digitized with the same 16-bit A/D converter that samples the vector DC electric field data. The design of the digital converters expressly enabled the three components of the electric field and the three components of the magnetic field to be sampled precisely simultaneously.

The magnetometer included an active thermal control to maintain a sensor temperature within the bounds of 5 to $25 \mathrm{deg} \mathrm{C}$ and was situated at the base of sensor on the boom mount, as shown on the right hand side of Fig. 30. The heater uses pulse-width modulation to drive $\mathrm{AC}$ currents in the resistive elements at higher frequencies outside the bandpass of the sensor to avoid magnetic contamination. It operated on a thermostat controlled by the magnetometer drive board that was part of the VEFI electronics stack. Temperature measurements were provided as routine state-of-health data and formed an important part of the instrument offset determination in the magnetometer data analysis.

The sensor is mounted on the end of a boom with low thermal conductivity that was deployed on a single hinge. The boomlength was $60 \mathrm{~cm}$ and was used to minimize the stray magnetic fields associated with the space vehicle. The boom, as shown in Fig. 32, consisted entirely of Zelux material provided by the VEFI team. Zelux is non-magnetic and has excellent thermal and mechanical properties. Whereas Goddard magnetometers had used Zelux on previous missions (e.g., Cluster, Mars Global Surveyor, Lunar Prospector, Wind, ACE, DMSP), this was the first time that we used $40 \%$ glass content, which made it stronger that the ones used in previous missions, which were 10-20\% glass.

Only non-magnetic materials were used in the magnetometer construction, including Lexan, aluminum, and titanium. The sensor cable, provided as part of the magnetometer, had a mass density of $60 \mathrm{~g} / \mathrm{m}$. The entire assembly was wrapped in a thermal blanket to help maintain a constant temperature inside the magnetometer housing and to minimize temperature-dependent measurement errors. 
Fig. 32 Photograph of the $60 \mathrm{~cm}$ magnetometer boom made entirely of Zelux with $40 \%$ glass shown above a drawing of the magnetometer boom stowed along the side of the spacecraft

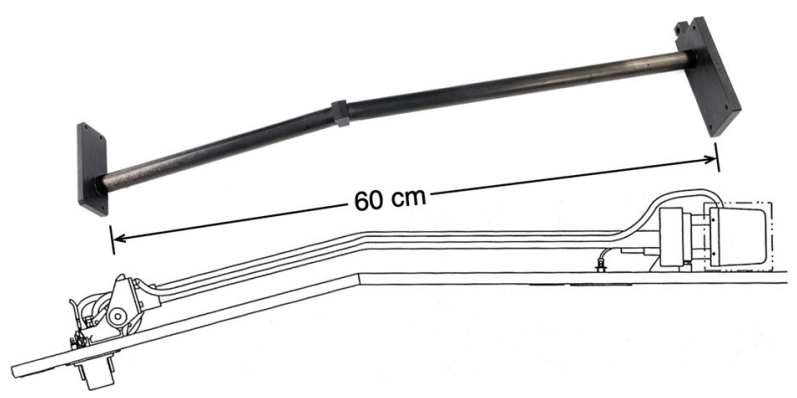

The entire magnetometer (including the drive board) required power of $0.5-0.7$ Watts, with the sensor requiring $60 \mathrm{~mW}$ on the boom. The sensor mass was $275 \mathrm{~g}$ (not including the thermal blanket).

The unit was calibrated prior to launch in the Goddard Magnetic Field Test Facility. Independent non-orthogonality measurements were carried out on a magnetically quiet day. The Earth's ambient, mid-latitude magnetic field was measured in each of the three orthogonal directions. Non-orthogonality and calibration procedures were also performed in space, during the entire 7.5 year C/NOFS mission, as described in Freudenreich and Pfaff (2021).

Although the accuracy of models of the Earth's magnetic field have improved in the last decade (e.g., POMME model, CHAOS model), in some regions, such as those of the south Atlantic, the magnetic field vector is not known to an accuracy better than $250 \mathrm{nT}$. This was particularly true when the C/NOFS mission was in formulation in the late 1990's. For reference, for a satellite such as C/NOFS orbiting at $8 \mathrm{~km} / \mathrm{sec}$ at low latitudes, a $\mathbf{V} \times \mathbf{B}$ induced electric field of $1 \mathrm{mV} / \mathrm{m}$ corresponds to a magnetic field of $125 \mathrm{nT}$ perpendicular to the velocity, and a $0.1 \mathrm{mV} / \mathrm{m}$ field corresponds to $12.5 \mathrm{nT}$.

The inclusion of a sensitive flux-gate magnetometer on the C/NOFS spacecraft drove magnetic cleanliness requirements stipulating that the non-geophysical magnetic field due to the spacecraft at the sensor location shall not exceed $125 \mathrm{nT}$. To achieve this, the solar arrays were "backwired" to minimize currents, and non-magnetic materials were used in the spacecraft wherever it was practical. These requirements were not onerous, and the general ambient or "DC" magnetic environment due to the spacecraft met the requirements and was acceptable. Nevertheless, large magnetic perturbations were observed due to torque rod firings, activity of the momentum wheel, and other sources. Although they were detrimental to the measurements, these discrete perturbations were largely deterministic, relatively shortlived, and could generally be identified in the data and removed. More challenging were step-like variations of a few nT, which corresponded to slight changes in the offset of some of the components, and typically occurred at night and at the terminators, as discussed by Freudenreich and Pfaff (2021).

AC Magnetic Field Measurements AC or wave vector magnetic field components were gathered by passing the same DC magnetic field components through a bandpass filter with gain. The resulting three AC magnetic field components are digitized with 16 bits at $15.879 \mathrm{~s} / \mathrm{sec}$ with $\pm 900 \mathrm{nT}$ dynamic range, providing a least significant bit corresponding to $0.02 \mathrm{nT}$. For the AC magnetic field data, the input had a gain of 50 at the center of the passband and a high pass, one pole filter at $0.05 \mathrm{~Hz}$ and a low pass, filter at $5 \mathrm{~Hz}$, as shown in Fig. 31. The vector magnetometer data could also be digitized at higher rates in the burst memory as either DC or AC-coupled channels. 


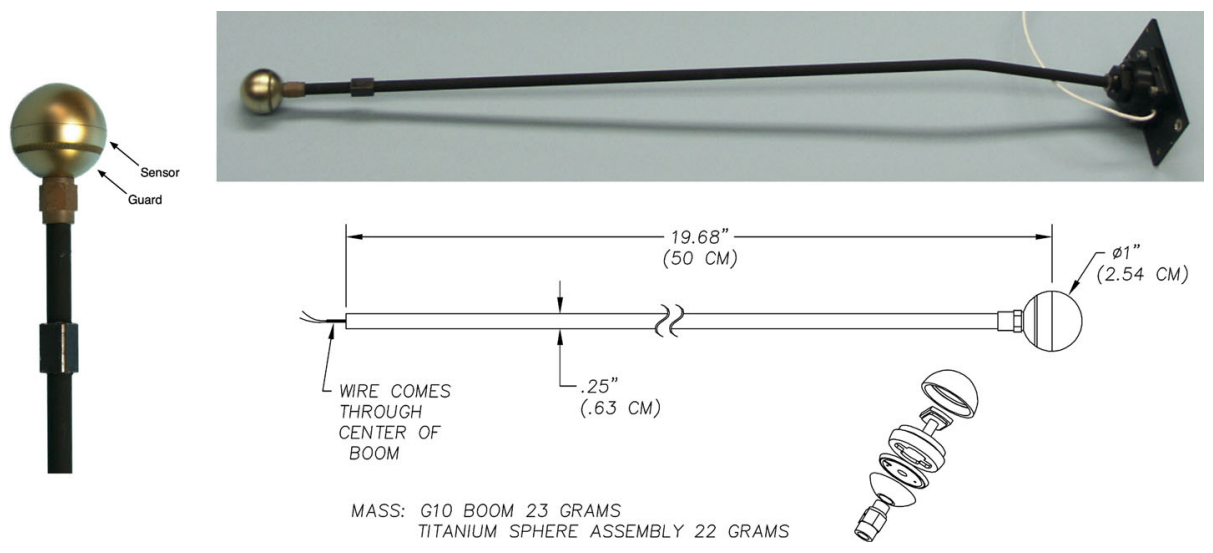

Fig. 33 Photograph of the VEFI fixed bias Langmuir probe showing the Titanium-Nitride coated sensor and guard (left). The probe is shown on the $50 \mathrm{~cm}$ deployable boom on the upper right, with a diagram on the lower right providing details

\section{Fixed-Bias "Fast" Langmuir Probe}

The VEFI Langmuir probe was designed to measure plasma density with a range of $10^{2}-10^{7} \mathrm{~cm}^{-3}$ at high time resolution. The VEFI relative plasma density measurements served as a back-up to the Planar Langmuir Probe on the spacecraft, built by the Air Force Research Laboratory (Roddy et al. 2010), which consisted of a swept Langmuir probe and an ion trap. The main function of the VEFI relative density fixed-bias probe was to provide input to be used to trigger the VEFI burst memory, identifying plasma depletions for high resolution spread-F irregularity studies. Accordingly, the VEFI fixed-bias probe was often referred to as the "Trigger Probe".

The principles of obtaining plasma density from the collected current on an orbiting spacecraft are provided by Brace (1998), and were used in Langmuir probes developed at Goddard and successfully flown on the DE-2, AE, and Pioneer Venus satellite probes.

The VEFI Langmuir probe consists of a $2.5 \mathrm{~cm}$ diameter sphere on a $50 \mathrm{~cm}$ arm, which is deployed from the nadir face of the spacecraft to avoid density disturbances behind the vehicle and spacecraft plasma sheath effects, as shown in Fig. 29. The lower third of the spherical probe included a guard, kept at the same potential as the main probe or collector, to mitigate any adverse effects of the boom, as shown in Fig. 33. The sphere (collector and guard) was made from titanium and had a surface treating of titanium nitride provided by TiSurf (Uppsala, Sweden), which also provided the coating for the Cassini Langmuir probe (Jacobsen et al. 2009).

The boom to deploy the Langmuir probe was made of G-10 and was provided by the VEFI team. The boom and sphere assembly are shown in Fig. 33. The boom deployed using a spring-loaded single-hinge mechanism provided by the spacecraft. Delrin was used for the insulator between the upper and lower sphere sections as well as for the mounting nut. The fundamental frequency of the probe was $10.7 \mathrm{~Hz}$ (deployed).

Relative plasma density data are gathered in a fixed-biased mode to collect ion current. The Langmuir probe gathered positive currents at a fixed voltage at a level of $-1.25 \mathrm{~V}$, $-2.5 \mathrm{~V},-3.75 \mathrm{~V}$, or $-5 \mathrm{~V}$, which was selectable from the ground. For most of the mission, the Langmuir probe was operated at $-3.75 \mathrm{~V}$ fixed bias. The data are nominally sampled at $15.879 \mathrm{~s} / \mathrm{sec}$ but could also be sampled at $508.133 \mathrm{~s} / \mathrm{sec}$ (nominal ELF mode) along with 


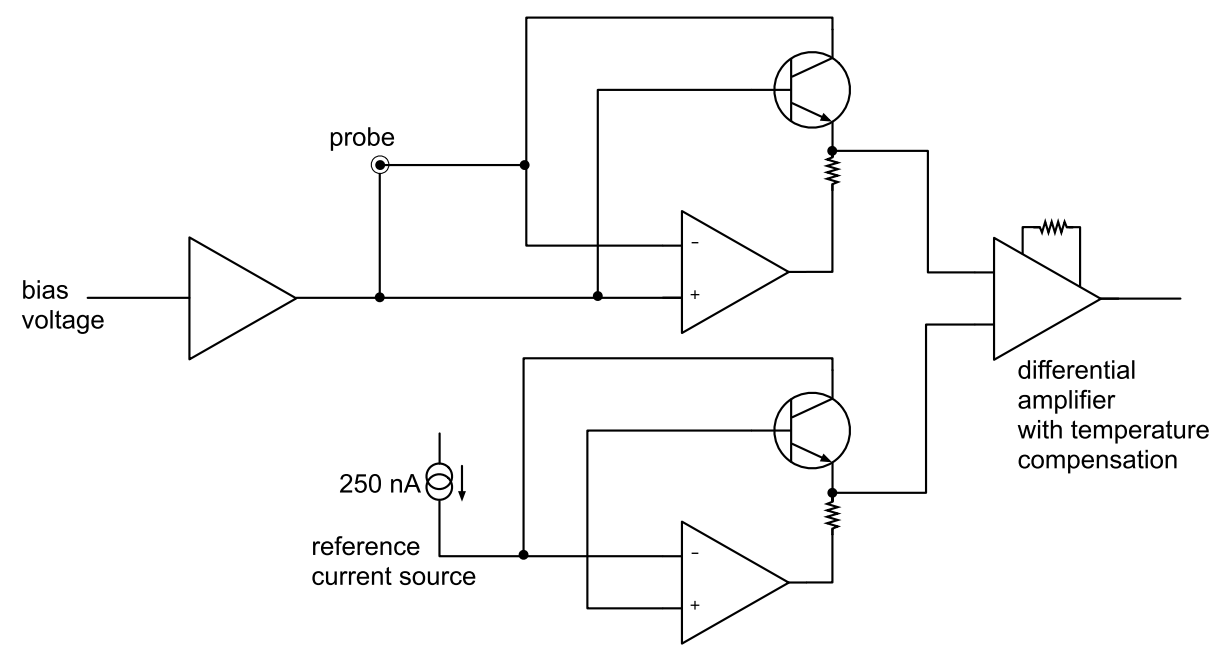

Fig. 34 Fixed-bias Langmuir probe circuit with temperature compensation
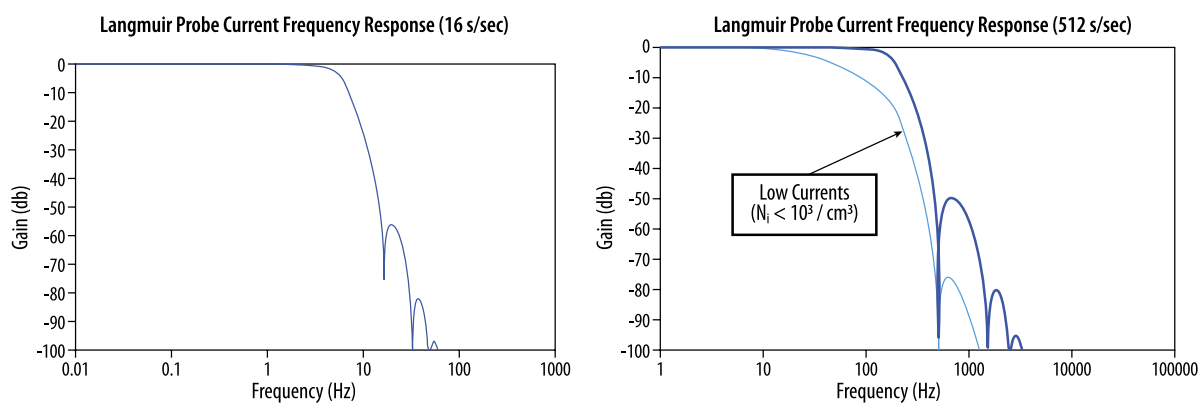

Fig. 35 Transfer functions of the low frequency $(16 \mathrm{~s} / \mathrm{sec})$ DC Langmuir probe channel (left) and the DC Langmuir probe with extended ELF response (right) which includes an attenuated frequency response for very low currents (densities)

one component of the electric field data, and at rates up to $32 \mathrm{ks} / \mathrm{sec}$ in the burst memory. The Langmuir probe was sampled with the same A/D converters that sampled the electric field data.

The Langmuir Probe block diagram is shown in Fig. 34, which shows a logarithm amplifier for the collected current, provided by a transistor. Because of the transistor's sensitivity to temperature, compensation is a necessity. The temperature compensation is achieved with a second, similar log amplifier, and a set reference current (as if it were a probe). Both transistors are assumed to be at the same temperature.

The transfer functions of the Langmuir probe frequency response are provided in Fig. 35. The frequency response varied for low current (low density). The transimpedance gain (from probe input current to output voltage) varies with the current magnitude. When the probe current is low, the gain is high. This results in a degraded frequency response, as the parasitic capacitances (the diode in particular) remain almost constant. As shown in the figure, for very low densities $\left(<10^{3} / \mathrm{cm}^{3}\right)$, the frequency response is attenuated. In other words, the circuit behaves like a $32 \mathrm{~Hz} 1$-pole low-pass filter for a $40 \mathrm{pA}$ current and a $16 \mathrm{kHz} 1$-pole 
Fig. 36 Optical lightning detector "footprint" for the north and south-oriented sensors along the spacecraft track
Optical Lightning Sensor "Footprints"

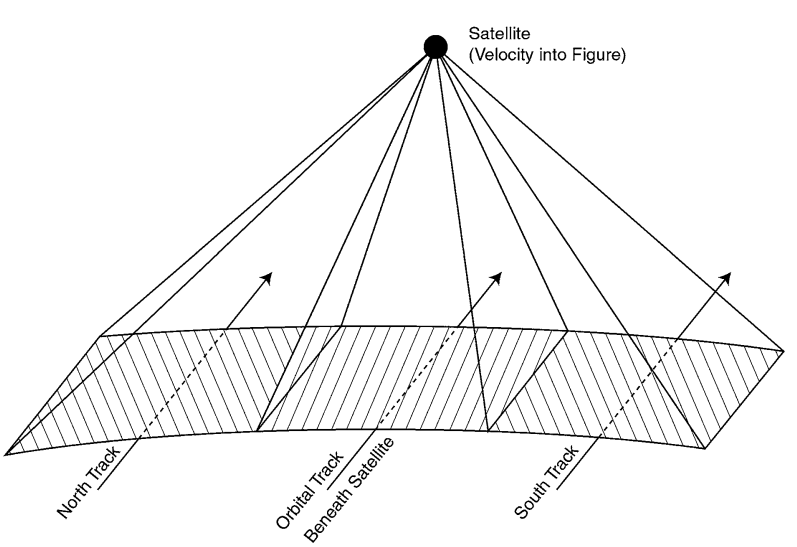

low-pass filter for currents above $20 \mathrm{nA}$. The transition from one response curve to the other is a smoothly varying function of current.

As discussed further on below, the conversion to plasma density of the collected ion current is dependent on the relative density of the ambient ions (see Klenzing and Rowland 2012).

\section{Lightning Detector}

VEFI includes an optical lightning detector (LD) for which the design, development, and prototype were provided by Drs. R. Holzworth and M. McCarthy at the University of Washington. This instrument recorded lightning count rates within specified levels, as well as raw optical lightning waveforms in a dual-channel instrument oriented north and south, as shown in Fig. 36.

The primary reason for including the lightning detector as part of the VEFI instrument is to distinguish electric field irregularities in the equatorial ionosphere that are driven by lightning. For example, lightning is a primary indicator of the source phenomena which may lead to explosive Spread-F (Woodman and Kudeki 1984) and other lightning induced phenomena (e.g., Kelley et al. 1985, 1990; Holzworth et al. 1985, 1999).

The overall design of the lightning detector which flew on C/NOFS is discussed in Jacobson et al. (2011). The detector had been flown previously on stratospheric balloons (Holzworth et al. 1986), sounding rockets (Li et al. 1991), and on the Blackbeard/Alexis satellite. In addition to recording lightning counts, the raw lightning detector signal could be digitized in the burst memory and used to trigger the burst memory wave capture.

The lightning detector included a two-sector field of view with one photodiode sensor per sector, and thus two optical channels consisting of one analog output per channel. Figure 37 shows the Lightning Detector fields of view of the two sensors. The actual sensor housing was milled from an aluminum block.

As discussed in Jacobson et al. (2011), the optical lightning sensor uses two independent silicon photodiodes $\left(44 \mathrm{~mm}^{2}\right.$ area each) oriented to look toward the nadir (i.e., toward the Earth), with one offset to the north and one to the south. The field of view is approximately 10 degrees of longitude by 20 degrees of latitude in both the north- and south-looking directions. The LD broadband input channel gathers optical power from $3 \mathrm{pW}$ to $70 \mathrm{nW}$ over the 
Fig. 37 Photographs of the lightning detector in which one of the optical photo-diodes is visible on the photograph on the right

Fig. 38 Chart showing the different amplitude levels for the lightning detectors
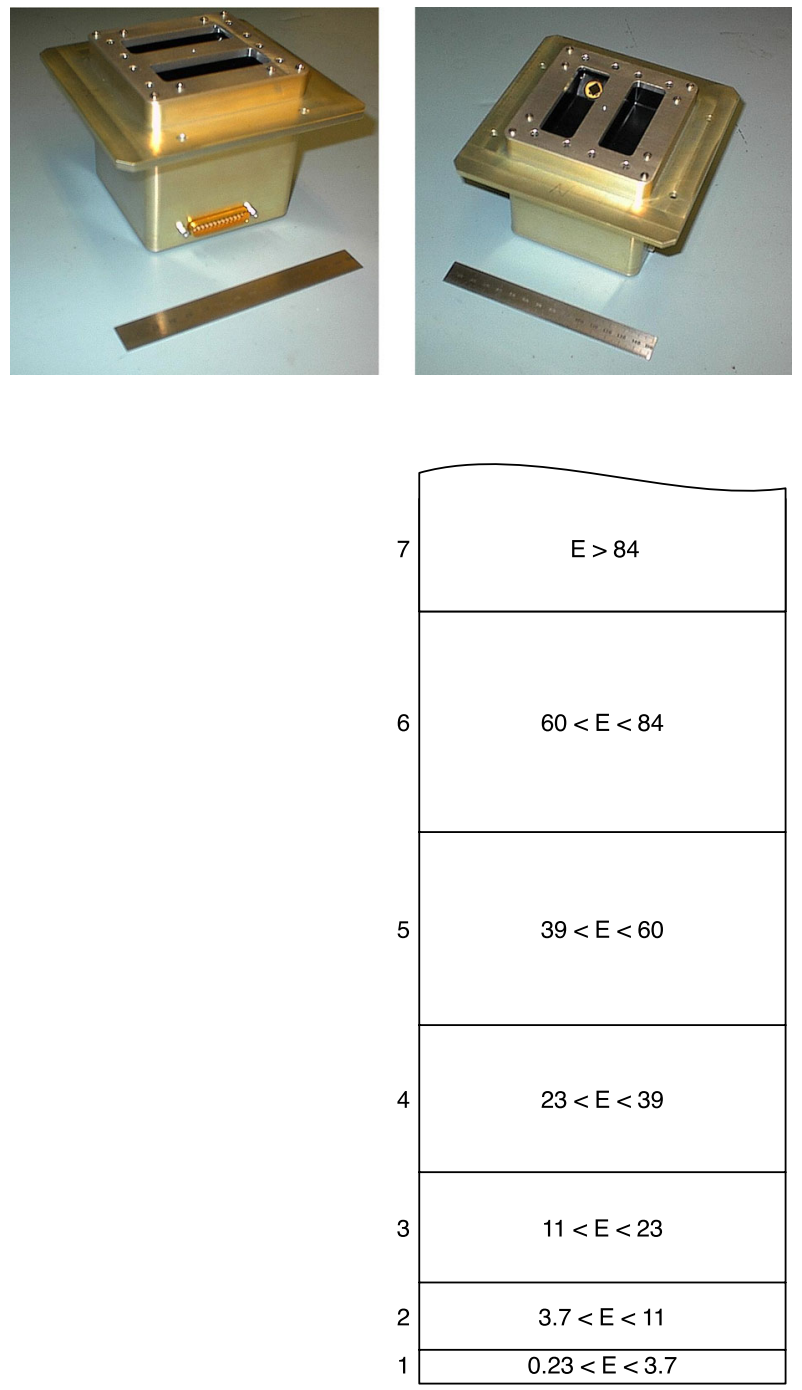

optical band defined by the silicon photodiode response of $400 \mathrm{~nm}$ to $900 \mathrm{~nm}$. The LD is designed with a passband that eliminates slowly varying optical power below about $0.3 \mathrm{~Hz}$. On-board comparators and counters for each channel enabled background contributions to be automatically removed. The data were binned into 7 different intensity levels as shown in Fig. 38.

The LD has two primary modes for data collection: low-time-resolution "counts" and continuous data collection in burst mode. In the low-time-resolution mode, which is available continuously, the instrument samples optical power at seven optical power levels for each photodiode, counting the time each channel is in each of the given power levels. These counts are telemetered every $0.5 \mathrm{~s}$ for each of the north- and south-looking LD detectors. In the burst mode, the optical power level from both the north and south LDs are sampled up to $32 \mathrm{ksamples} / \mathrm{s}$, along with other VEFI instruments such as the electric field waveforms. 


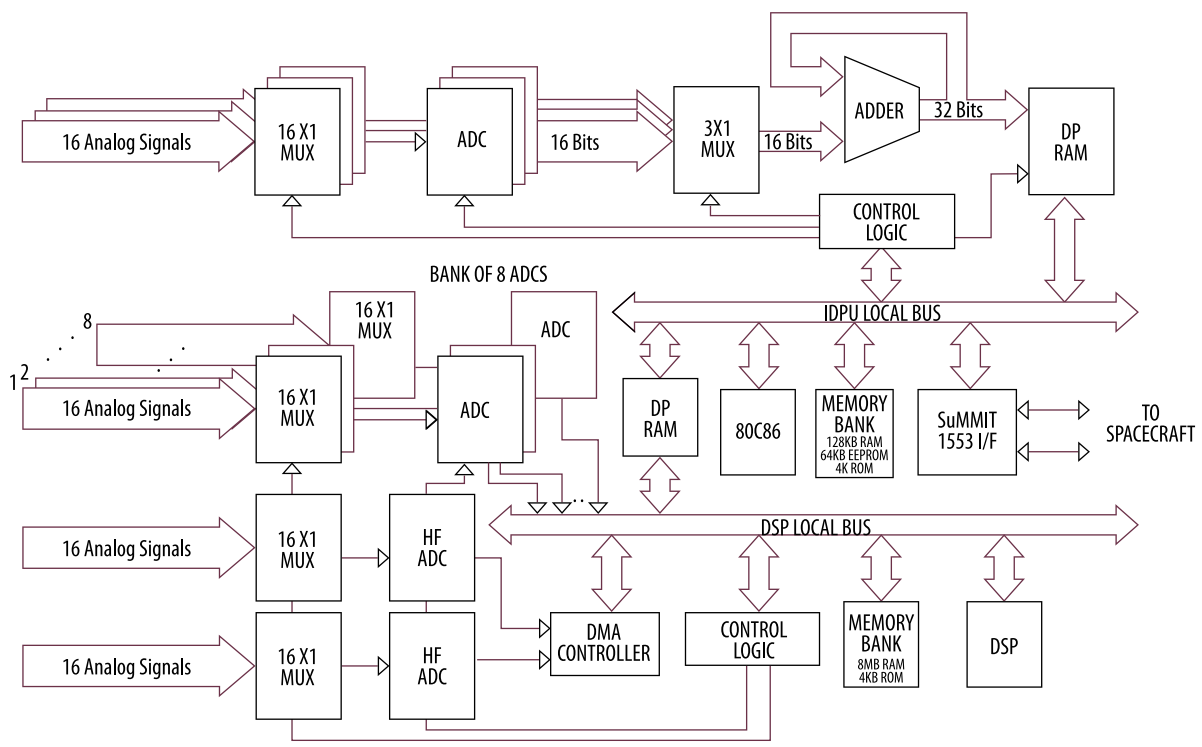

Fig. 39 Digital electronics block diagram

\section{Digital Architecture, Flight Software, Commands, and Operations}

Overview The VEFI digital architecture is divided into two units: The Instrument Data Processing Unit (IDPU) and the Digital Signal Processor (DSP). The IDPU was the core of VEFI as it received and executed commands from the ground, gathered the science data, formatted it, and transmitted it to the spacecraft Command and Data Handling (C\&DH). The DSP enhanced the functionality of the VEFI by computing the Fast Fourier Transform (FFT) on the data as well as implementing digital filtering and the burst memory functionality. The IDPU flight software ran on an 80C86 microprocessor while the DSP flight software ran on a $320 \mathrm{C} 25$ microprocessor.

A detailed block diagram of the VEFI digital architecture showing the different banks of ADCs, the MUXes, and the IDPU and DSP local buses is provided in Fig. 39. This diagram includes the memory and control logic as well as the 1553 interface to the spacecraft.

\subsection{IDPU Software}

The IDPU software was broken into two parts: the bootstrap loader and the main program. When power was first applied to VEFI, the IDPU began executing the bootstrap loader software stored in PROM. This program fit within $1.5 \mathrm{kB}$ and initialized the 1553 interface, RAM, FPGAs and various internal variables. After initialization, the software checked if the current start-up was caused by the application of power (cold start) or by a ground command or a watchdog timer reset (warm start). The bootstrap loader would abort the automatic boot process and wait for operators to manually load the flight software after 16 consecutive warm starts. It is worth noting that during the entire C/NOFS mission, there was only one VEFI warm start caused by the watchdog timer.

The IDPU main flight software managed all of the activities of the instrument. Due to power constraints it ran on the $80 \mathrm{C} 86$ clocked at $4.8 \mathrm{MHz}$. Because some of the tasks required deterministic timing execution, it was written in assembly language and ran without 
the aid of an operating system. Real time tasks were executed inside an interrupt service routine while command and telemetry routines ran in the foreground. The IDPU software was very compact, occupying only $6 \mathrm{kB}$ of memory, and implemented the following tasks:

- Continuosly sample 48 analog signals at different sampling rates.

- Transfer the DSP data (FFT and burst memory) using DMA.

- Produce state of health (SOH) telemetry.

- Format the real time, DSP, and SOH data and transmit that data to the S/C.

- Implement three different modes of operation: Slow Survey, Nominal Survey, and Fast Survey.

- Decode and execute commands.

- Perform Burst Trigger algorithm.

- Time tag the data with an absolute time accuracy of $1 \mathrm{~ms}$.

\subsection{DSP Software}

The DSP flight software executed on the $320 \mathrm{C} 25$ microprocessor running at $24 \mathrm{MHz}$ with $8 \mathrm{MB}$ RAM and $4 \mathrm{~KB}$ ROM. It managed all of the higher speed signal processing tasks that the IDPU could not handle and was in charge of the burst memory system. It was also written in assembly language for speed and also ran without the aid of an operating system.

The DSP implemented a special 512-point Radix-4/Radix-2 real-FFT algorithm to transform 1024 data samples for the survey mode FFTs. This algorithm transformed the data using radix-4 butterflies in the first stages and a final radix-2 butterfly stage completed the transform. The reverse indexing required to order the data was complex as not only binary but also quaternary bit-reverse addressing was required. The benefit of using this algorithm was the speed of execution as it took less than half the time a 1024-point radix-2 FFT algorithm took. The data was pre-processed by a window selected from the ground (rectangular, Hamming, or Hanning).

The burst memory software was very flexible and could be configured to capture from one to 255 bursts of one to eight channels of data, with each channel having eight different selectable inputs, and variable sampling rates from $2 \mathrm{kHz}$ to $32 \mathrm{kHz}$, and having a burst memory pre-cursor of variable length. This flexibility introduced complexity in the allocation of the $8 \mathrm{MB}$ memory. A separate mode could allow the burst memory to sample the two HF ADCs at $8 \mathrm{MHz}$. The burst memory is further discussed below.

\subsection{State of Health}

VEFI included comprehensive state of health $(\mathrm{SOH})$ and housekeeping data packets that include temperature measurements and raw data from each sensor, digitized with VEFI's 16-bit Crystal 5016 at $1 \mathrm{~s} / \mathrm{sec}$, as well as currents and voltages and other verifiers of the state of the instrument. The SOH data was sent to the spacecraft and transmitted to he ground at variable rates, but never higher than $1 \mathrm{~s} / \mathrm{sec}$. The $\mathrm{SOH}$ data was also kept in the VEFI science data header at $1 / 32 \mathrm{~s} / \mathrm{sec}$.

\subsection{Telemetry and Operations}

VEFI transmitted data to the $\mathrm{S} / \mathrm{C}$ through the 1553 interface. The forecast and survey data streams were transmitted using time division multiplexing and the S/C took care of piping those data streams to their corresponding transmitters (see Fig. 40). The forecast data was 
Fig. 40 Diagram showing the different VEFI downlink telemetry modes
Telemetry: Downlink Modes

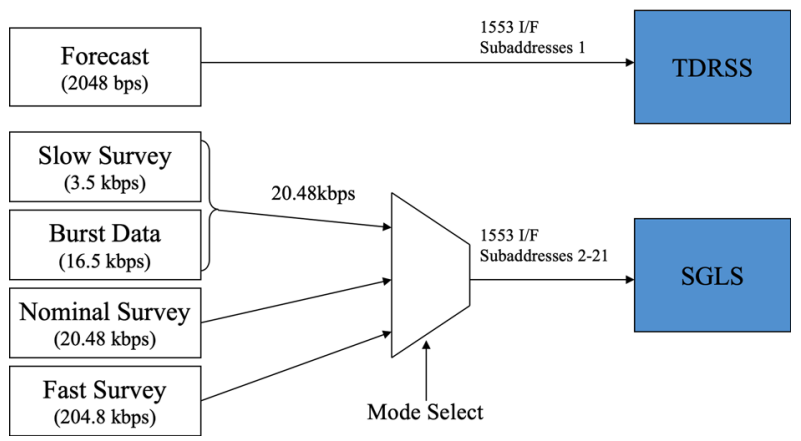

a $2.048 \mathrm{kbps}$ subset of the survey data while the latter also contained three sub modes: slow (3.5 kbps), nominal (20.48 kbps), and fast survey (204.8 kbps). Table 2 shows the science data that was transmitted in each of the modes. The fast survey mode was a special high telemetry rate provided by the spacecraft at certain intervals during the mission. The forecast (TDRSS) mode was a low-rate mode for real-time operations.

Because the scintillation-producing irregularities are a nighttime phenomenon, VEFI operated in slow survey during the daytime portion of the orbit and nominal survey and burst mode collection occurred at night. See Fig. 9 for a diagram showing the operations for a typical orbit. The shift to fast survey occurred 5 minutes prior to sunset and continued to 5 minutes past sunrise.

During the day, the $20.48 \mathrm{kbps}$ survey mode consisted of $3.5 \mathrm{kbps}$ "slow survey" and $16.5 \mathrm{kbps}$ download of the VEFI burst data acquired during the previous nighttime portion of the orbit. During the nighttime, VEFI collected (and transmitted to the spacecraft) both slow survey data and the two-channel broadband ELF data.

\section{Programmable Burst Memory}

VEFI includes a programmable burst memory to capture high resolution (up to $32.768 \mathrm{ks} / \mathrm{sec}$ ) 16-bit data "snapshots" of time-domain DC- and AC-coupled electric fields, plasma density, magnetic field, and optical lightning data. The data can be sampled up to $32.768 \mathrm{k} \mathrm{sam}$ ple/sec ( $\sim 0.25 \mathrm{~m}$ spatial resolution) and stored in the instrument's 8 Mbyte dedicated burst memory in response to programmable triggering. The number of bursts per orbit, channels per burst, sample rate per channel and duration of burst are configurable by command. All data are 16 bits.

The VEFI Burst memory gathers high resolution data from selected channels when interesting events are detected. The burst memory is programmed to "trigger" on a variety of different parameters that could be selected in advance. Triggers include:

Electric Field Wave Power (from the 12-channel filter bank)

Plasma Density Depletions

Lightning Flashes

Pre-assigned Time 
Table 2 VEFI data channels showing their specific bit rate allocation for forecast, slow survey, nominal, and fast survey data modes
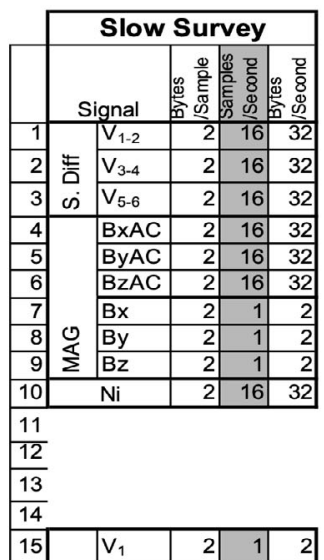

\begin{tabular}{|l|l|l|l|l|l|}
\hline 15 & & $V_{1}$ & 2 & 1 & 2 \\
\cline { 1 - 4 } 16 & & 2 & 1 & \\
\hline
\end{tabular}

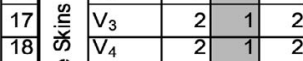

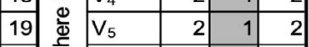

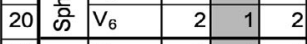

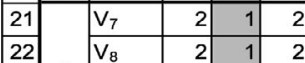

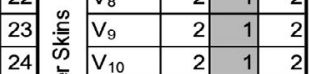

\begin{tabular}{|l|l|r|r|}
\hline 25 \\
\hline \multirow{2}{*}{ के }
\end{tabular}

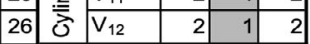

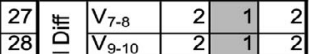

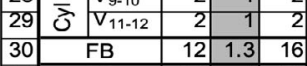

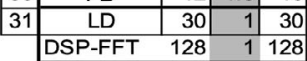

\begin{tabular}{rrrr} 
DSP-FFT & 128 & 1 & 128 \\
\hline Total: & & 434
\end{tabular}

*can substitute for either $V_{3-4}$ elf or $V_{5-6}$ elf
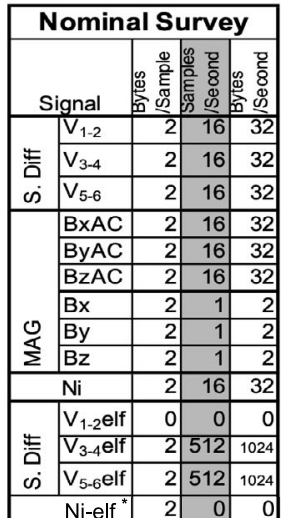

\begin{tabular}{|c|c|c|c|c|}
\hline \multirow{6}{*}{ 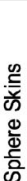 } & $V_{1}$ & 2 & 1 & 2 \\
\hline & $V_{2}$ & 2 & 1 & 2 \\
\hline & $V_{3}$ & 2 & 1 & 2 \\
\hline & $V_{4}$ & 2 & 1 & 2 \\
\hline & $V_{5}$ & 2 & 1 & 2 \\
\hline & $V_{6}$ & 2 & 1 & \\
\hline
\end{tabular}

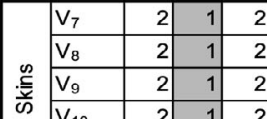

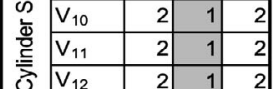

\begin{tabular}{|l|l|r|r|r|}
\hline \multirow{2}{*}{ 纴 } & $V_{7-8}$ & 2 & 1 & 2 \\
\cline { 2 - 4 } & $V_{9-10}$ & 2 & 1 & 2 \\
\hline
\end{tabular}

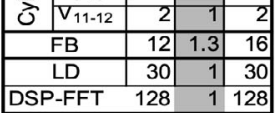

Total: 2482
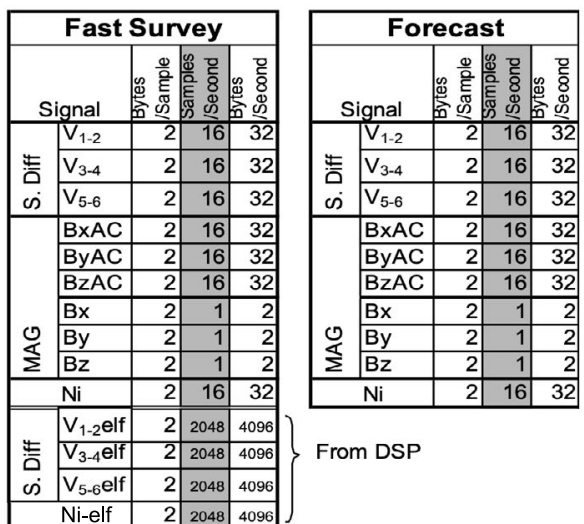

From DSP

\begin{tabular}{|c|c|c|c|c|c|c|c|c|c|}
\hline & | II-en & $<$ & 048 & 1096 & & & & & \\
\hline \multirow{6}{*}{$\begin{array}{l}\frac{\mathscr{2}}{5} \\
\text { के } \\
\frac{\Phi}{\Phi} \\
\frac{0}{0}\end{array}$} & $V_{1}$ & 2 & 1 & 2 & \multirow{6}{*}{ 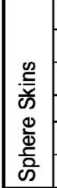 } & $V_{1}$ & 2 & 0.5 & 1 \\
\hline & $V_{2}$ & 2 & 1 & 2 & & $V_{2}$ & 2 & 0.5 & 1 \\
\hline & $V_{3}$ & 2 & 1 & 2 & & $V_{3}$ & 2 & 0.5 & 1 \\
\hline & $V_{4}$ & 2 & 1 & 2 & & $V_{4}$ & 2 & 0.5 & 1 \\
\hline & $V_{5}$ & 2 & 1 & 2 & & $V_{5}$ & 2 & 0.5 & 1 \\
\hline & $V_{6}$ & 2 & 1 & 2 & & $V_{6}$ & 2 & 0.5 & \\
\hline
\end{tabular}

Trigger criteria included plasma density depletions detected by an onboard algorithm provided as part of the VEFI software, electric field wave power amplitude from any of the 12 selectable filter bank channels, optical lightning detector irradiance, a combination of these, or a selected pre-determined time. Examples of pre-selected times correspond to when the $\mathrm{C} / \mathrm{NOFS}$ satellite was in conjunction with a ground-based radar or in conjunction with a burn of the Space Shuttle.

The trigger algorithm embedded in VEFI flight software continuously scores events that are encountered; the bursts with the highest scores are subsequently telemetered to the ground. The highest scoring burst data are kept in separate memory and only the final, highest scoring data are then output to the spacecraft on the next orbit.

VEFI's burst memory provided a special "wave interferometer" mode that enabled the measurement of the phase velocity and wavelength of waves. It did this by gathering "spaced receiver" phase-difference measurements between opposing co-linear double probes, formed from the potential difference of a cylinder sensor and its nearby sphere sensor, or by determining the wave vector direction from the $\mathrm{dE}$ vector obtained in the orbit plane from single-sphere potential outputs. A typical low-rate selection might include four 
bursts of 120 second duration each during a nighttime pass, consisting of 4 channels at $2048 \mathrm{~s} / \mathrm{sec}$ triggered on spread-F plasma depletions.

The burst modes include the following variables. These variables were selected via command and were same for all bursts in a given orbit:

\author{
\# Bursts per orbit \\ \# Channels per burst \\ Sample rate per channel (same for all channels in burst) \\ Duration of burst \\ Duration of the precursor time within a burst
}

A common group of low-rate burst memory data captured at $2048 \mathrm{~s} / \mathrm{sec}$ is:

$$
\text { V12, V34, V56, Ni }
$$

Higher-rate selections might include 2 bursts of 15 second duration during a nighttime pass, each consisting of 4 channels.

Common groups of high-rate burst memory data captured at $32768 \mathrm{~s} / \mathrm{sec}$ are:

VLF12, VLF34, VLF56, Ni; or

VLF34, VLF56, LD1, LD2; or

VLF3-9, VLF4-10, VLF5-11 and VLF6-12 (interferometer channels)

Burst signals are sampled at $32.768 \mathrm{kHz}$ by eight 16-bit A/D converters. These signals could be downloaded at slower rates, in which case the decimation takes place through a digital filtering process. Since the decimation rate varies during the mission, the analog antialiasing filter cutoff frequencies reflect the A/D sampling rate, not the download rate. The anti-aliasing performance is also affected by the digital filters.

The burst memory enabled 1-8 selectable channels at rates of 2048, 4096, 8192, 16384, and $32768 \mathrm{~s} / \mathrm{sec}$ each. The channel inputs were selectable among $4 \mathrm{DC}$ channels sampled at $32768 \mathrm{~s} / \mathrm{sec}$. These inputs might be a DC electric field, a magnetic field, or optical photometer outputs from the dual-channel lightning detector. Additionally, $4 \mathrm{AC}$ or waves channels were available that included either a DC-coupled channel with a boosted AC response or an AC-coupled broadband data with a bandpass filter. The frequency response of these different channels is shown in Figs. 41. The fixed-bias Langmuir probe data could also be captured in the high-rate burst memory, either DC coupled or in a mode with the higher frequencies boosted. The frequency response of these channels is shown in lower portion of Fig. 41, and shows two curves that are functions of the detected current, as discussed in the fixed-bias Langmuir probe section above.

\title{
9 VEFI Coordinates, Electric Field Boom Straightness, and Spin Maneuvers
}

In this section, we provide measurements to illustrate the straightness of the electric field booms and their sensor positions. We also show an example of a C/NOFS spin maneuver. To interpret these observations, we begin by presenting the VEFI coordinate system.

\subsection{VEFI Coordinates}

The VEFI instrument evaluation and subsequent data processing and analysis utilize three sets of coordinates: spacecraft coordinates, geographic coordinates, and geomagnetic coordinates. These are discussed below. 

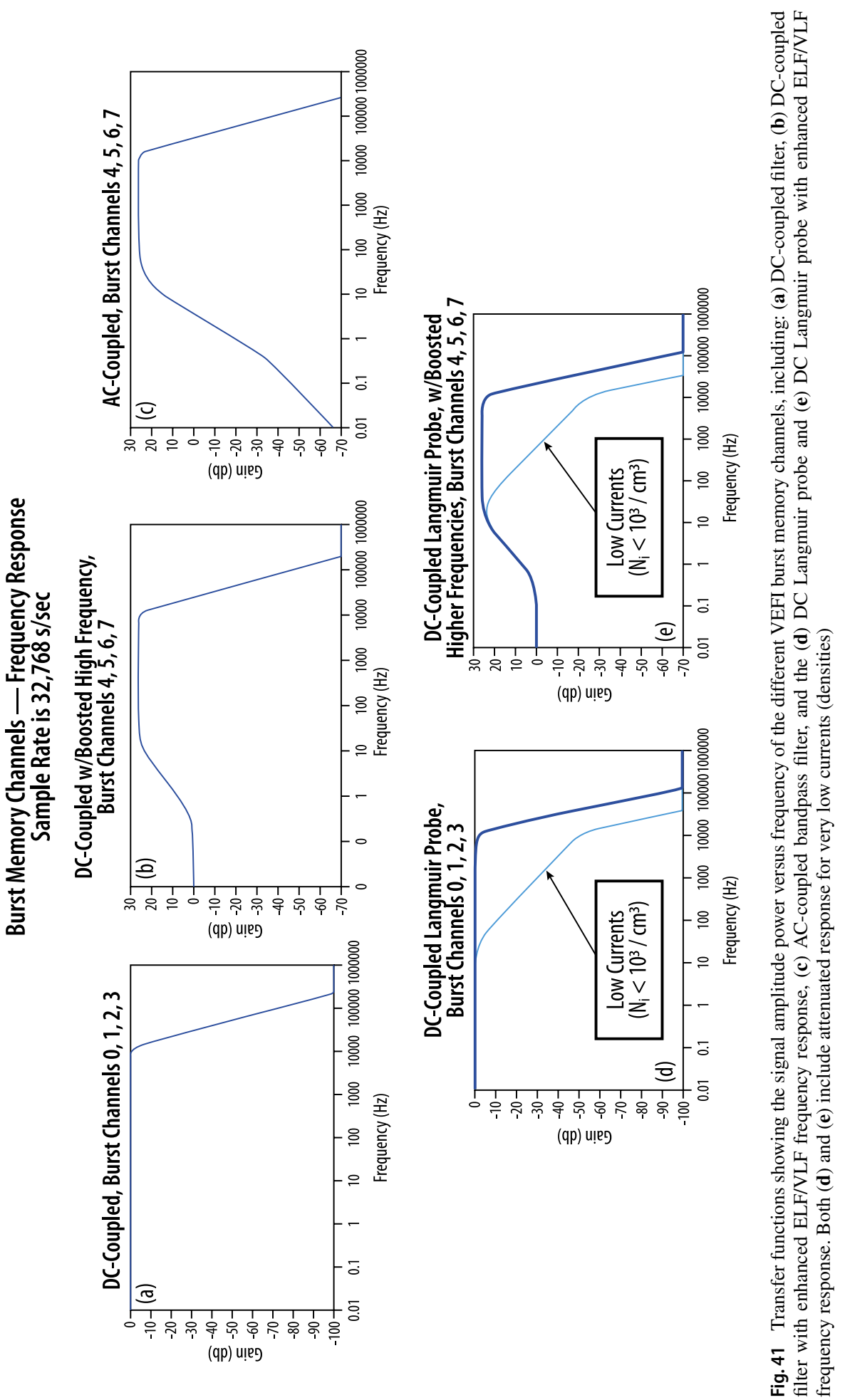

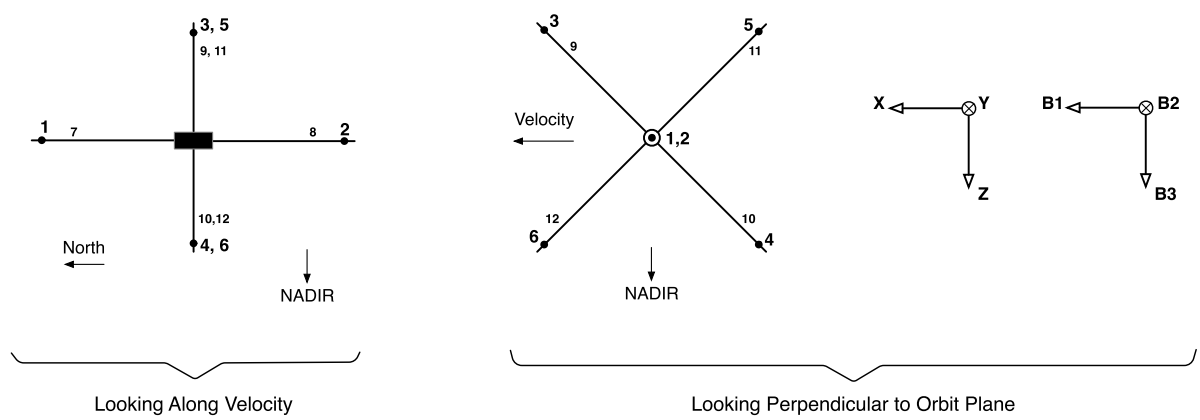

Fig. 42 VEFI coordinates on the spacecraft showing the nominal position of the sensors with respect to the orbit plane and spacecraft velocity

The C/NOFS spacecraft coordinates are defined by the orthogonal directions inherent to the spacecraft itself, as shown in Fig. 42: X, from the center of the spacecraft in the nominal ram direction (generally eastward); Y, perpendicular to the plane of the orbit (generally southward); and Z, nominally toward the nadir.

Four electric field booms, as discussed previously in Fig. 8, lie in the plane of the orbit, or the X-Z plane, but are rotated 45 degrees from the $\mathrm{X}$ direction. These are the " $3-4$ " and "5-6" booms as shown in Fig. 42, in which spheres 3 and 6 lead the spacecraft and 4 and 5 trail. The third electric field component is generally perpendicular to the orbit and is along the Y direction. It is represented by the " $1-2$ " boom with sphere 1 in the nominal north direction and sphere 2 in the nominal south direction.

The VEFI magnetometer is deployed in the aft or trailing portion of the spacecraft, as described previously (see Fig. 29). Its three orthogonal components, B1, B2, and B3, correspond to $\mathrm{Bx}, \mathrm{By}$, and $\mathrm{Bz}$.

A three-dimensional rendering of the electric field booms is shown in Fig. 8. The direction of each of the three electric field components, E12, E34, and E56, is described as follows. As discussed earlier, each component of the electric field is measured as a potential difference between opposing spheres. As $\mathbf{E}=-\nabla \Phi$, the measured potential differences between spheres a and $\mathrm{b}$ correspond to an electric field component directed from a to $\mathrm{b}$. Accordingly, the E12, E34, and E56 measurements correspond to the directions from spheres 1 to 2 , spheres 3 to 4 , and spheres 5 to 6 .

The geographic coordinate system is the standard "local vertical/local horizontal" reference frame defined by the three orthogonal directions: north (x), east (y) and vertical down (z). This reference frame is co-rotating with the Earth.

Another coordinate system which is important for the VEFI data analysis is the geomagnetic reference frame. This coordinate system will be encountered later in this article when representative science data are shown. Essentially, after gain, offsets, and non-orthogonality corrections are made, the VEFI vector measurements (both electric field and magnetic field data) are rotated from spacecraft coordinates to a geophysical coordinate system for scientific analysis and comparison with other measurements. In the case of the VEFI fields data, a useful geophysical coordinate system is that defined by the earth's ambient magnetic field, or local geomagnetic coordinates, as shown in Fig. 43.

Figure 43 depicts the earth's dipole magnetic field, for which the magnetic poles are shifted from the geographic poles by about 10-15 degrees, and hence the geomagnetic equator is also shifted accordingly. With its 13-degree-inclination orbit and initial apogee and perigee of 400 and $850 \mathrm{~km}, \mathrm{C} / \mathrm{NOFS}$ measurements are gathered entirely within the low 

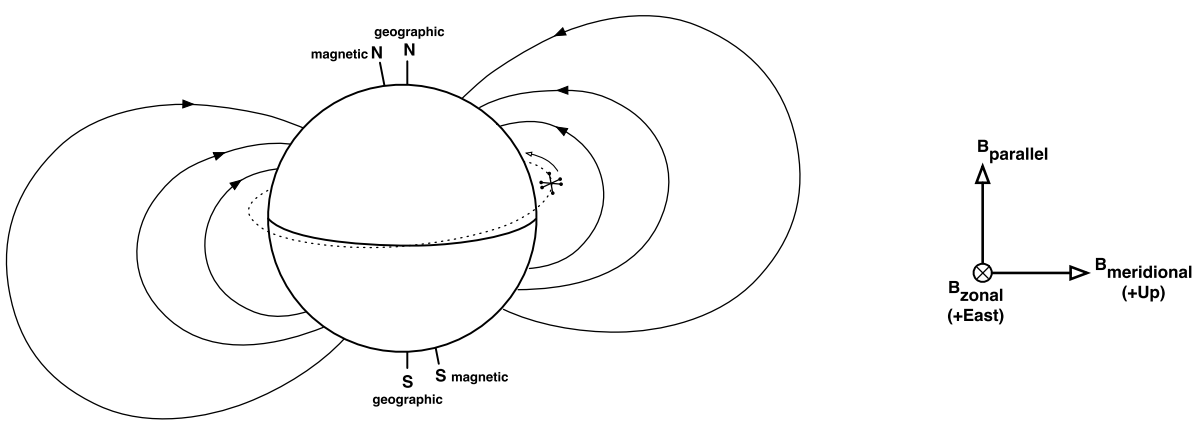

Fig. 43 Magnetic coordinates defined by the earth's dipole magnetic field at low latitudes

latitude, closed dipole magnetic field region. Hence, at all altitudes along the C/NOFS orbit throughout its 7.5 year mission, the primary magnetic field direction (defined as B-parallel in Fig. 43) is generally in the north direction. We now define a coordinate system about the local magnetic field vector such that the zonal direction is defined by $\mathbf{B} \times \mathbf{R}$, where $\mathbf{B}$ the local magnetic field vector and $\mathbf{R}$ is the vector from the center of the Earth to the spacecraft. The zonal component is positive in the eastward direction. The meridional component is the zonal direction $\times \mathbf{B}$ and is positive outward. Note that at the magnetic equator, the (positive) meridional component is essentially in the (upward) vertical direction.

\subsection{Observed Electric Field Boom Straightness and Orthogonality}

An important requirement for a successful DC electric field experiment is that the booms maintain their orthogonality and straightness and that the positions of the sensors are thus known with a required level of precision. This enables the sensor potential differences to be transformed from the spacecraft frame to the geophysically relevant frames and to ensure the full vector is measured with high accuracy. This is particularly important at low and mid latitudes where the ambient electric field is on the order of $1 \mathrm{mV} / \mathrm{m}$ and the $\mathbf{V} \times \mathbf{B}$ contribution is typically several hundred $\mathrm{mV} / \mathrm{m}$. Slight deviations from orthogonality of the components in their fully deployed sensor configuration can be determined by establishing a non-orthogonality matrix at regular intervals during the mission as afforded by examination of the measurements themselves in conjunction with concurrent, highly accurate spacecraft attitude knowledge. Possible deviations might occur due to slight bending of the booms resulting from neutral density forcing or thermal variations, including possible changes between daytime and nighttime conditions on each orbit, such as when the satellite crosses the terminator.

As discussed previously, the six VEFI electric field booms were precisely situated on the spacecraft such that when fully deployed, the intersection of the opposing spheres along the three double-probe axes form three mutually orthogonal components. This required canting the booms when they were mounted on the spacecraft body to account for the physical offset of the deployers on the spacecraft platform. Shims were used to negate the slight, reproducible, inherent bending of each antenna element, as discussed earlier. With the use of alignment mirrors, the boom unit orientations at launch were known with high precision and a nominal non-orthogonality matrix was thus available for the initial electric field data analysis. During the C/NOFS mission, the actual alignment and orthogonality of the measurement axes were checked using several methods, including the following: 
(a) Comparison of the measured potential differences with the requirement that there be no DC electric fields along the magnetic field direction or that $\mathbf{E} \cdot \mathbf{B}=0$.

(b) Comparison of the measured potential differences with the assumption that the measured averaged DC electric fields were those of the $\mathbf{V} \times \mathbf{B}$ fields.

(c) Examination of the measured spacecraft shadow location when one sphere of a double probe pair was eclipsed by the spacecraft during the day, and a resulting distinct signature of photoelectron imbalance was observed in the electric field data.

We now examine each of these methods in turn with respect to the actual C/NOFS VEFI observations.

(a) Definitive information regarding the orthogonality of the three double probes can be obtained by examining whether the measured potential differences met the requirement that there be no DC electric fields along the magnetic field direction or that $\mathbf{E} \cdot \mathbf{B}=0$. This analysis was carried out during the early part of the mission and was used to confirm the general orthogonality of the tri-axial electric field measurement directions.

(b) Another approach to pinpoint the locations of the electric field sensors consisted of equating the measured potential differences to $\mathbf{V} \times \mathbf{B}$, which comprise more than $99 \%$ of the detected signal, except, perhaps, during large spread-F depletions at night. Here, we assume that the measured averaged DC electric fields, integrated over a large number of orbits, were represented by those of the $\mathbf{V} \times \mathbf{B}$ fields. Here, the very small amplitude ambient fields would be negligible, or, when averaged over all local times, extremely small. These assumptions are valid at low latitudes on a low inclination satellite in low earth orbit such as C/NOFS, even during active conditions.

This analysis was treated as an optimization problem with 21 free parameters: the $\mathrm{X}, \mathrm{Y}, \mathrm{Z}$ positions of the six spherical probes, in spacecraft coordinates and the additive offsets in the $\mathrm{X}, \mathrm{Y}$, and $\mathrm{Z}$ components. Because the instrument offsets and electric fields may vary with local time, the analysis was run separately as a function of local time for different times of the mission.

The data used in the analysis shown here consisted of almost 4 years of data from August 8, 2008 to June 9, 2012. Starting from the nominal boom lengths and orientations, $\mathbf{E}-\mathbf{V} \times \mathbf{B}$ was input to an optimization program that found the position of each sphere that minimized $\mathbf{E}-\mathbf{V} \times \mathbf{B}$ for that sphere. The program was constrained in that it would allow the boom to bend, effectively getting shorter, but not to stretch. If a sphere was too far from the origin, the program applied a penalty to the figure of merit, guiding the sphere inward.

Using this condition, we found the optimal physical displacement of the spheres for one day (15 orbits) at a time. The results are shown in Fig. 44. The red rectangles mark the interquartile range $\left(1.35^{*}\right.$ standard deviation) of these points in two dimensions. All distances can be converted to degrees by atan[X or Y or Z]/boomlength.

The analysis shown in Fig. 44 reveals that the predicted sphere locations deviated on the order of 10's of cm or less, in support of a non-orthogonality of less than 1 degree. Importantly, the analysis shows that the predicted displacement for all 6 sensors showed similar results. To investigate systematic variations with local time and also with altitude and solar activity, including possible thermal-induced bending, the boom straightness was analyzed in a similar fashion for other times during the mission. For all cases, the general sphere locations were shown to be consistent with the statistical distribution shown in Fig. 44. In particular, we determined that there were no observed differences between the sphere positions during the daytime and nighttime.

(c) Spacecraft occultations occur when the sphere of one double probe enters the spacecraft shadow. This is an excellent means to both verify the sensor position, as well as the 
C/NOFS Electric Field Probe Positions

August, 2008 to June, 2012 -- 9:30 - 17:30 LT

White Circle: Nominal Position; Red: Mean, Interquartile Range
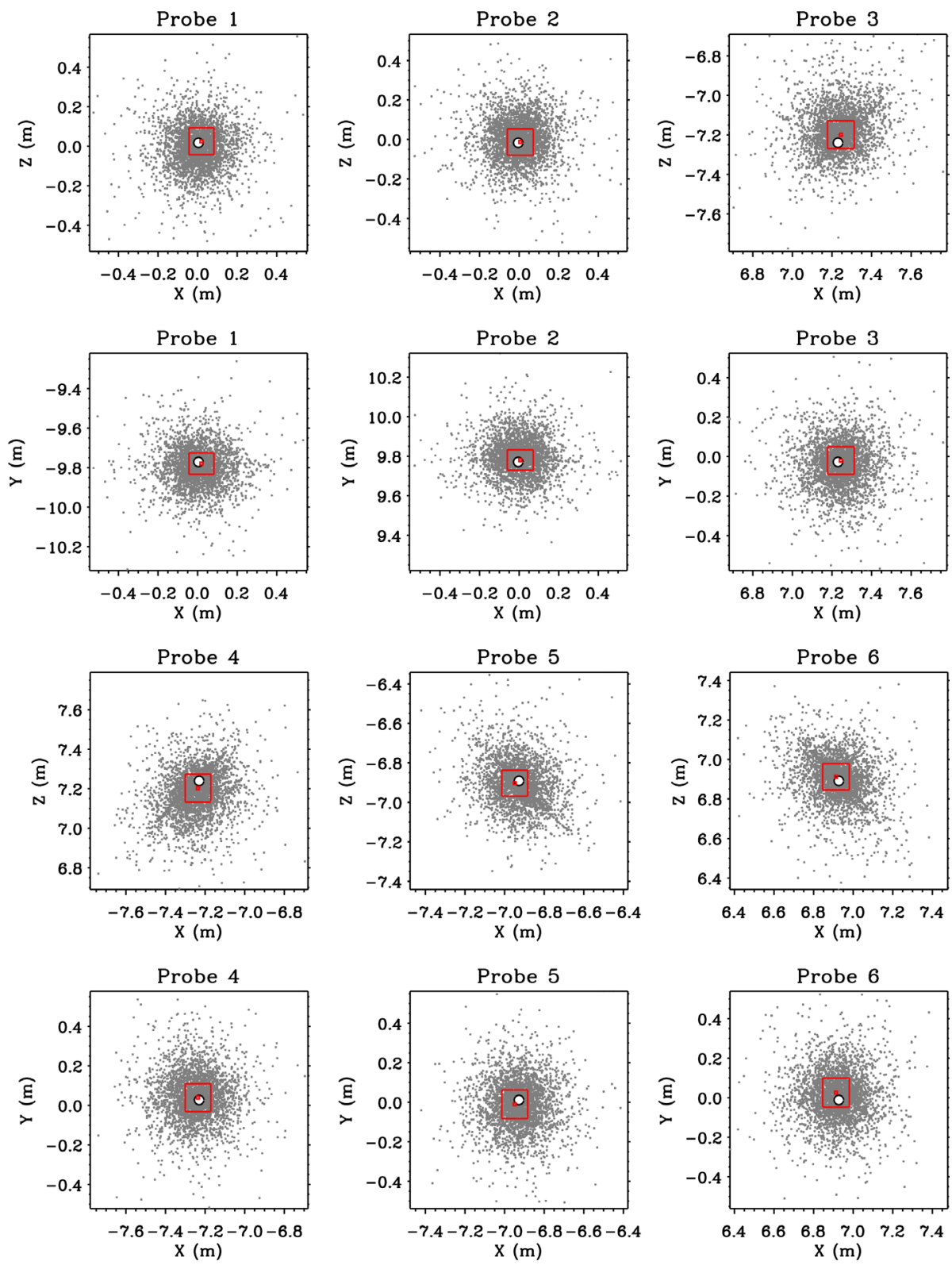

Fig. 44 Variations of the VEFI electric field probe positions in spacecraft positions deduced from the measurements during the period from August 2008 to June 2012 
Fig. 45 Diagram showing the nominal positions of the VEFI electric field double probes in the orbit plane with respect to the sun showing the local times in which spacecraft shadowing might occur for certain solar angles
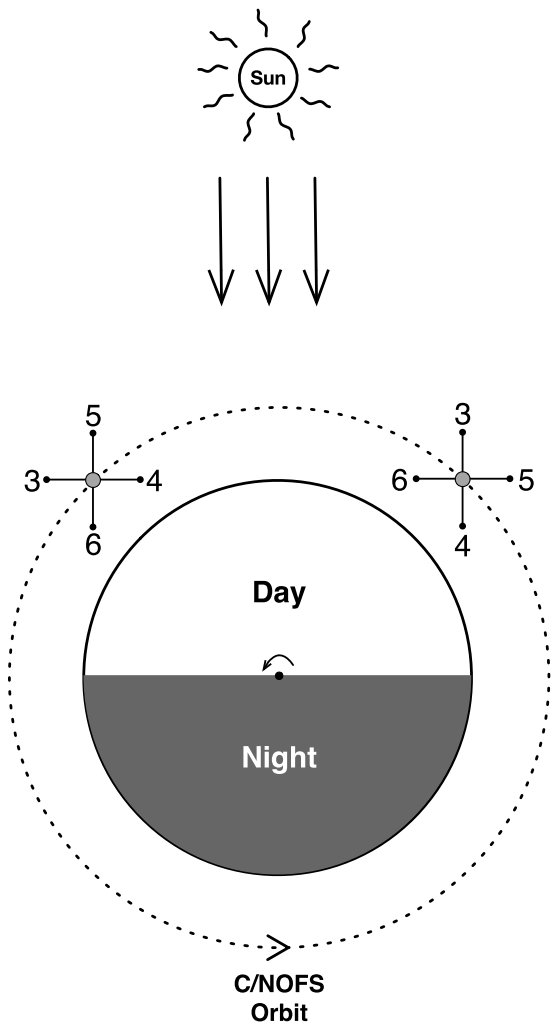

attitude information. For C/NOFS, the occultation of a sensor by the spacecraft shadow occurred during intervals when the sun-ecliptic angle was less than the C/NOFS inclination of 13 degrees. As illustrated in Fig. 45, this condition occurred for the probes in the orbital plane in the morning and afternoon whenever the orbit plane was aligned with the sunspacecraft direction. This occurred in the morning, near 9 LT when the "3-4" double probe was aligned with the sun and sphere 4 went behind the spacecraft, and near 15 LT when the "5-6" double probe was aligned with the sun, and sphere 6 went behind the spacecraft into shadow.

Figure 46 shows a series of double probe potentials from August 24, 2008, plotted against the azimuth of the Sun in the XZ plane (180 degrees is C/NOFS “noon"). A spike marks the occultation of a sensor. They occur at 225 degrees and 315 degrees, 90 degrees apart, as expected. These observations not only confirm that the boom orthogonality was within one degree of expectation (assuming the spacecraft $\mathrm{X}$-axis was perfectly aligned with the ram direction), but their reproducible offsets provide definitive information regarding the precise sensor locations. Note that the duration of the shadow depends on the aspect ratio of the boom length and spacecraft diameter.

Figure 47 shows an ensemble of measured electric field variations due to the sunspacecraft shadows for the first and third eclipse periods of the mission. Note that the shadows appeared consistently at their expected locations with respect to the solar azimuth, with the center of the 5-6 shadow off by about 1 degree, providing information on the \#6 sphere location in the $\mathrm{X}-\mathrm{Y}$ plane, and the center of the 3-4 shadow off by about 0.5 degree, providing information on the \#4 sphere location in $\mathrm{X}-\mathrm{Y}$ plane. 
Fig. 46 Electric field measurements from the " $3-4$ " and "5-6" double probes on August 24, 2008, plotted versus solar azimuth showing the spacecraft shadow effects (depicted by the dashed lines)
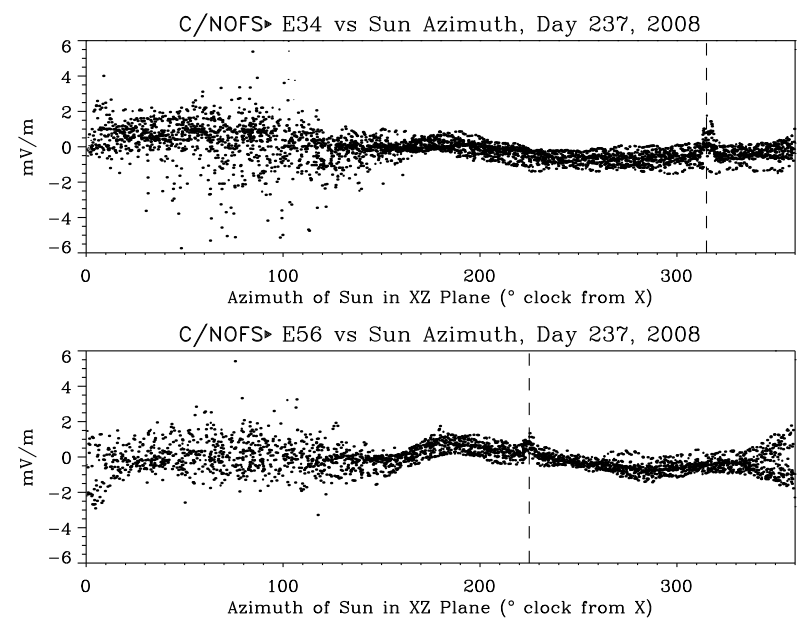

The observed angular offset of the solar elevation angle was due to the asymmetry of the boom positions on the satellite. Since the C/NOFS satellite is considerably extended in one direction with respect to the center line of the booms, the shadow effects were skewed. This is shown in the spacecraft sketches in Fig. 47 for the 3-4 and 5-6 booms. In fact, since the 5-6 boom was positioned slightly below that of the 3-4 booms, the elevation angles of the two shadow geometries differed by approximately 1.5 degree. This small difference is discernible upon careful inspection of the actual shadow angles in enlargements of the data (not shown). This result confirms that the sphere locations were in their expected positions within the $\mathrm{X}-\mathrm{Z}$ plane. Furthermore, we remark on the silhouette of the spacecraft shadow in azimuth. The width of the overall shadow signature is approximately 6-7 degrees and corresponds to the aspect ratio of the satellite diameter (about 1 meter) to the boomlength of $9.5 \mathrm{~m}$. The wider extent of the lower portion of the shadow reflects the greater diameter of the lower portion of the spacecraft due to its extended solar array skirt, as shown on the diagrams in Fig. 47. This is particularly evident in the E34 data shown for days 885-1249 on the upper right hand panel. The remarkable similarity of the shadow geometry and the spacecraft profile supports our interpretation that photoelectron shadowing is a useful tool to interpret the precise sphere locations.

Note the faint line that extends from the center in elevation in Fig. 47. This is believed to be the shadow of the "1-2" boom system that may be considered an extension of the spacecraft body that ultimately eclipses the shadowed sphere when perfectly aligned with the sun direction. Since the boom diameter is $2.86 \mathrm{~cm}$, the shadow from the boom element would be expected to be far less than a degree, but here glint and other effects may account for the observed width of $\sim 1$ degree of this feature.

We now extend this analysis for the entire C/NOFS mission, as shown in Fig. 48. The upper panel of this figure shows the elevation of the sun in the spacecraft $\mathrm{X}-\mathrm{Z}$ plane for the entire 7.5-year C/NOFS mission. The figure clearly show that such eclipse "seasons" are present once a year during months centered on the equinoxes, as depicted by the dashed lines. Again, the dashed lines are offset from zero because the booms were not in the geometric center of the spacecraft.

The lower panels of Fig. 48 show the azimuth of the measured, mean sun-spacecraft shadow effects in the "3-4" and "5-6" double probe data which is accurate to within a few tenths of a degree. The specific azimuths assume that the ram direction is precisely at 0.0 degrees. Although there may be noise and non-shadow signal at the shadow locations which 

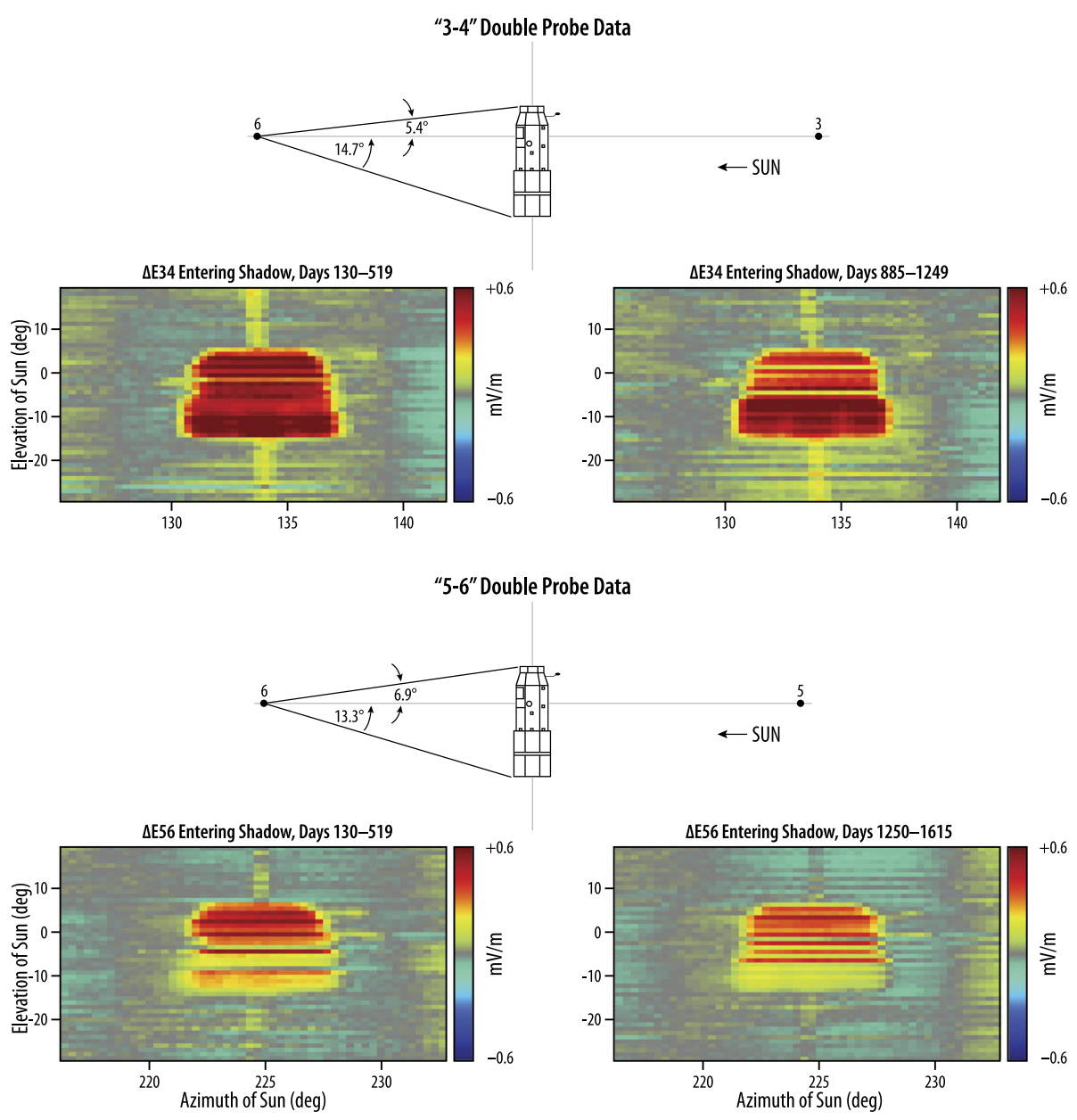

Fig. 47 Composite (averaged) measurements of the electric field variations measured by the "3-4" (top) and "5-6" (bottom) double probes for two different eclipse seasons plotted as a function of the solar azimuth and elevation. The diagrams above show the angles subtended by the spacecraft shadow for the two double probes

would affect the location of the centroid, in general the sun-shadow results shown here confirm that the electric field booms (or specifically, spheres 4 and 6) are in their expected locations for the duration of the C/NOFS mission.

The overall assessment of the observed VEFI electric field boom straightness and orthogonality is that the boom system maintained its expected configuration throughout the mission. The small variations that contribute to observed non-orthogonality matrices facilitate a more accurate DC electric field analysis. Small amplitude ( $\sim$ few tenths of a $\mathrm{mV} / \mathrm{m}$ ) level shifts were occasionally detected as the spacecraft travelled across the terminator. These are discussed in a more detailed assessment of the behavior of the electric field detector in Pfaff et al. (2021).

In addition to the measurements and analysis shown here, comparison of the phase difference of the two double probes in the spin plane, when the spacecraft rotated slowly in pitch on occasional, infrequent intervals, provided still further evidence that the double probes were in their nominal positions during the mission. This is discussed next. 

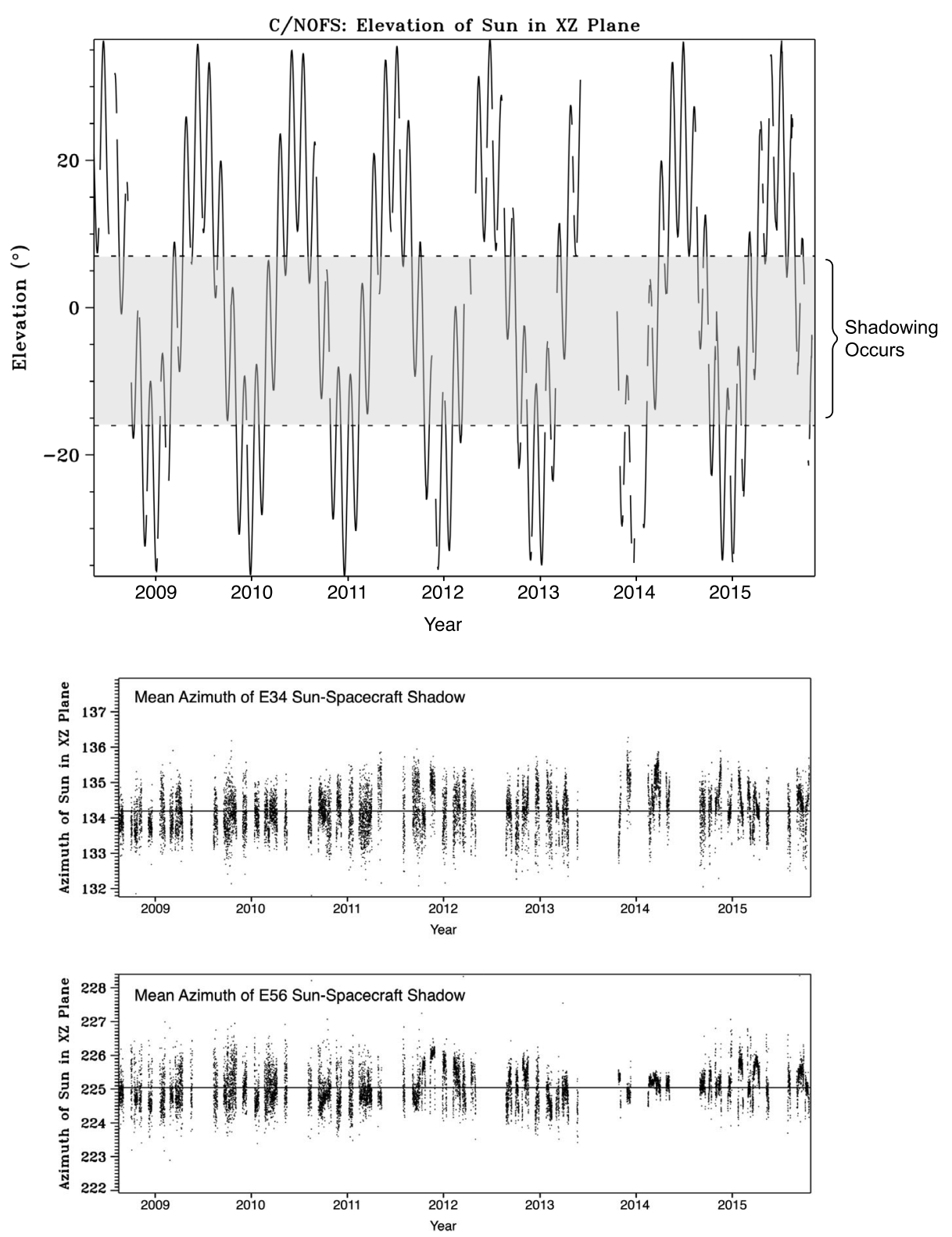

Fig. 48 Elevation of the sun in the spacecraft $X-Z$ plane for the entire 7.5-year C/NOFS mission (upper panel). The lower panels show the azimuth of the measured, mean sun-spacecraft shadow effects in the "3-4" and "5-6" double probe data

\subsection{Spin Maneuvers}

During the C/NOFS mission, the spacecraft occasionally underwent a series of very slow pitch spin maneuvers from which, among other objectives, the electric field and magnetic field offsets of their respective components in the spin plane/orbit plane could be readily 
Fig. 49 Electric field measurements corresponding primarily to $\mathbf{V} \times \mathbf{B}$ potentials from the triaxial electric field double probes during a spin test on May 20, 2008

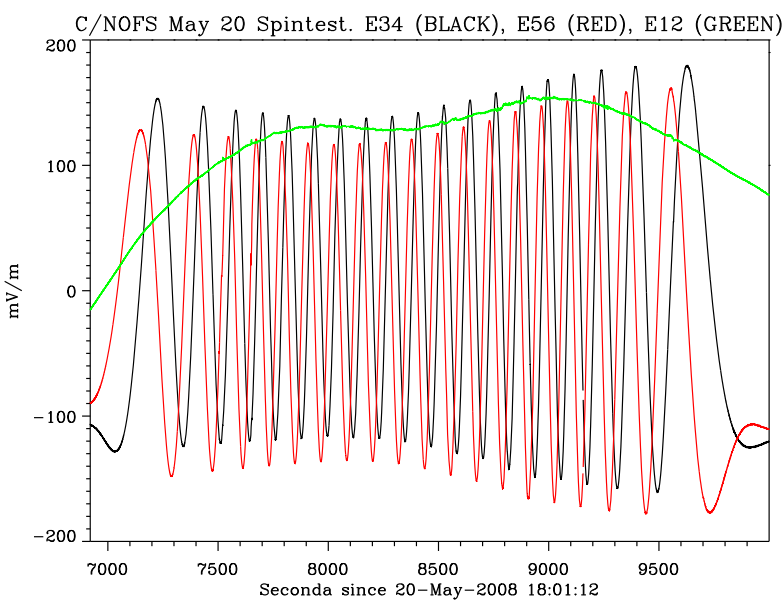

determined. An example of the raw electric field components, E12, E34, and E56 are shown in Fig. 49 for a spin maneuver on May 20, 2008. Note that the two orthogonal electric field components, E34 and E56, show a sinusoidal variation as they are in the "spin plane" whereas there is no such sinusoidal variation in E12 since, for these maneuvers, that double probe is along the spin axis. The figure shows that the E34 and E56 components are out of phase by 90 degrees, confirming their orthogonality. This 90 -degree phase shift was consistent for all spin maneuvers carried out during the mission.

The C/NOFS spin maneuvers also demonstrated that there was no noticeable wake effect in the electric field data when the 4 spheres in the spin plane rotated through the spacecraft wake. Such inspection was carried out at different altitudes, local times, and plasma density conditions, as discussed in Pfaff et al. (2021).

\section{Electric Field and Magnetic Field Processing and Examples of Data Collected from All VEFI Sensors}

\subsection{Electric Fields}

The processing of the VEFI electric field data gathered in the low earth orbit ionosphere followed standard procedures, such as those outlined in Maynard (1998) and Pfaff et al. (2010a). As described previously, the potential difference measurements provide the three components of the electric field vector along the spacecraft orbit. The data were converted to $\mathrm{mV} / \mathrm{m}$ using the gain and boomlength information and then non-orthogonality information was applied. Offsets and $\mathbf{V} \times \mathbf{B}$ electric fields were removed and the remaining "ambient" or geophysical electric fields were rotated from the spacecraft frame to geophysical coordinates for analysis and study. This ambient electric field consisted of both a DC and $\mathrm{AC}$ (or wave) component. We discuss the DC electric fields first.

\subsubsection{Offsets}

A key aspect of the DC electric field analysis involves identifying and removing offsets from each of the three orthogonal components. Such offsets vary slowly during the mission and 
are generally due to changes in the sensor surface properties and interactions with the plasma as well as changes in the surface properties (e.g., from photoemission or work function) of the sensors.

Examples of the three components of "raw" DC electric field data are shown in Fig. 50 for two different days of the C/NOFS mission. The measurements are represented by the black traces and the $\mathbf{V} \times \mathbf{B}$ electric fields, calculated for each component based on the attitude and positional data of the satellite, are shown in red. The data in Fig. 50 immediately show two important aspects of the DC electric field measurements that are critical for routine, successful electric field data processing on a low altitude, low latitude mission such as C/NOFS: First, the electric field components measured along the low inclination C/NOFS orbit exhibit quasi-sinusoidal variations for each orbit. Thus, although the spacecraft is not spinning, the large scale variations of the electric field components and their intrinsic offsets are readily discernible. Second, the majority of the signal amplitude is due to $\mathbf{V} \times \mathbf{B}$, which changes along the C/NOFS elliptical orbit as the earth's ambient magnetic field strength and direction change with longitude and altitude.

Importantly, the difference between the measurements and the $\mathbf{V} \times \mathbf{B}$ signals produce a noticeable offset that is very slowly varying. This can be seen by inspecting the difference of the measured fields and the $\mathbf{V} \times \mathbf{B}$ values for each component between the two days of data shown in Fig. 50 for an orbit in 2008 and an orbit in 2010. Furthermore, for the daily variations shown here, which comprise 15 orbits and hence all local times, the offsets are essentially constant and do not show any noticeable difference between day and night. The lack of a local time effect on the offsets can be readily discerned from examining the times the spacecraft was in and out of eclipse, as shown in the lowest panels in Fig. 50.

The offsets of the DC electric field components do change slowly over the course of the mission and considerable attention was given to determining these offsets and their slow variations. The offsets were determined using a number of techniques. Of the different approaches utilized by the VEFI team, we comment here on the ones that were the most successful. Ultimately, a combination of these different, independent techniques was used to verify the offsets.

(1) Assume $\mathbf{E} \cdot \mathbf{B}=0$

(2) Assume $\nabla \times \mathbf{E}_{\text {zonal }}=0$ around an entire orbit

(3) Find offsets during quiet periods assuming the measurements correspond to $\mathbf{V} \times \mathbf{B}$

(4) Utilize infrequent spin tests

The main, initial approach is to obtain average daily offsets for each orthogonal doubleprobe component assuming there are no DC electric fields along the magnetic field line (i.e., $\mathbf{E} \cdot \mathbf{B}=0$ ). This assumption is well-established for electric-field measurements in the ionosphere, particularly those gathered away from the high latitude region, and has been validated using detailed comparisons of double-probe and ion-drift measurements on previous satellites (Hanson et al. 1993). To this end, we assumed that the DC offset was constant over an orbit and then varied the offsets via an optimization program along the roughly 100minute orbit for the electric field data sampled at $1 \mathrm{sample} / \mathrm{sec}$ to determine the best fit. This was then repeated for all successive orbits using a half orbit overlap. Offsets derived using this method are shown for the first 1.75 years of the mission in Fig. 51.

An additional refinement of this approach that was included in our analysis is the assumption that the integrated zonal electric field is zero about a single orbit (i.e., $\nabla \times \mathbf{E}_{\text {zonal }}=0$ ). This assumption, valid for low latitude, low altitude missions such as C/NOFS, provided only slight adjustments to the offsets and was not used routinely. The " $\nabla \times \mathbf{E}_{\text {zonal }}=0$ " approach provided a means to validate our overall procedures. 
C/NOFS VEFI Electric Field "raw" data and V $x$ B

May 23, 2008 (Orbits 538-553)

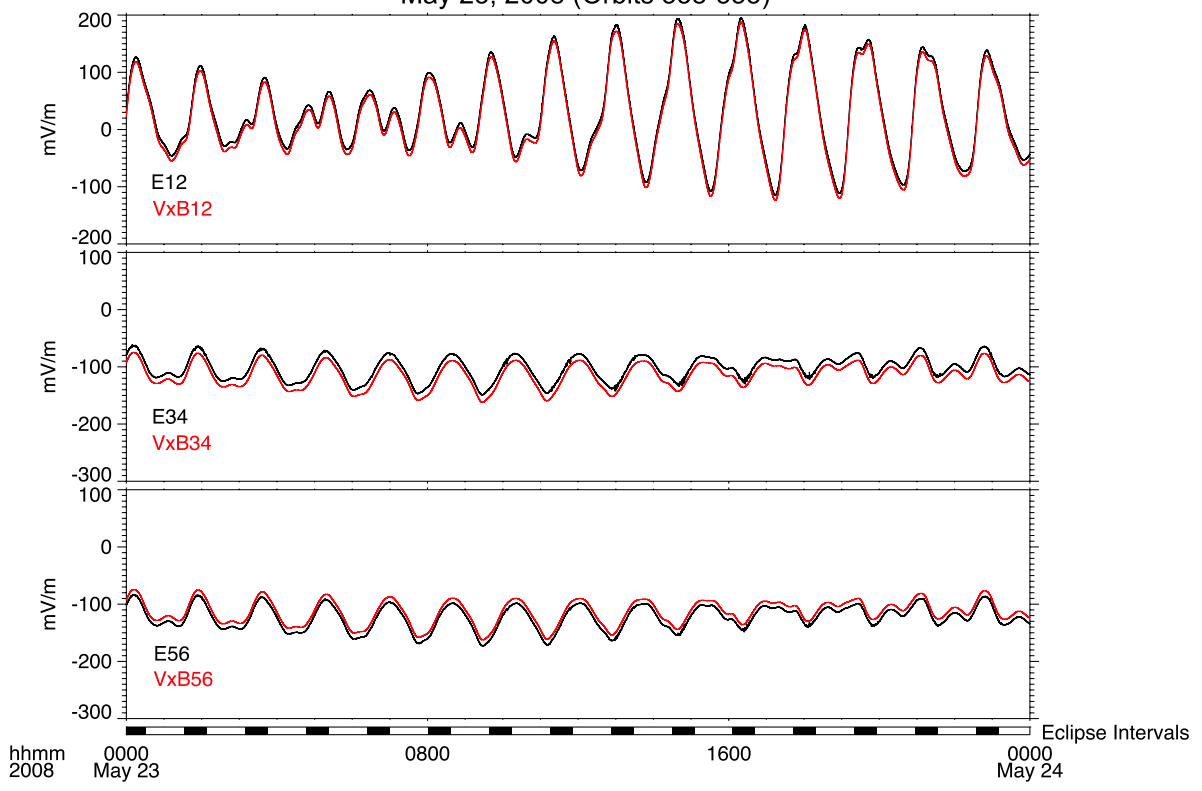

C/NOFS VEFI Electric Field "raw" data and V $x$ B

February 13, 2010 (Orbits 9908-9923)

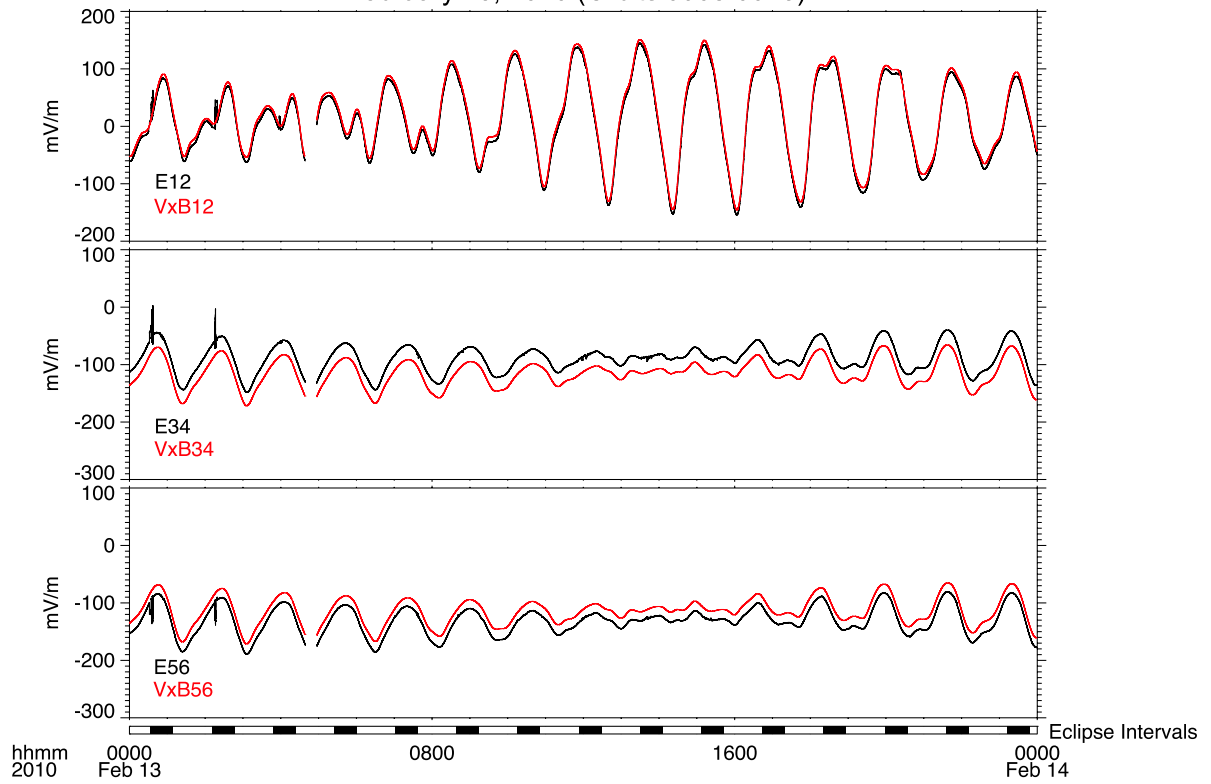

Fig. 50 Three components of "raw" DC electric field data (black) for two different days of the C/NOFS mission. The red curves show the $\mathbf{V} \times \mathbf{B}$ potentials calculated using the spacecraft position and attitude. The lowest panel shows the times in which the spacecraft was in eclipse. The offsets discussed in the text are the roughly constant vertical spacing between the red and black curves 
C/NOFS DC Electric Field Offsets Deduced from Different Techniques Black: V x B Comparisons, Red: "fits" using E•B = 0, Green: Spin Tests

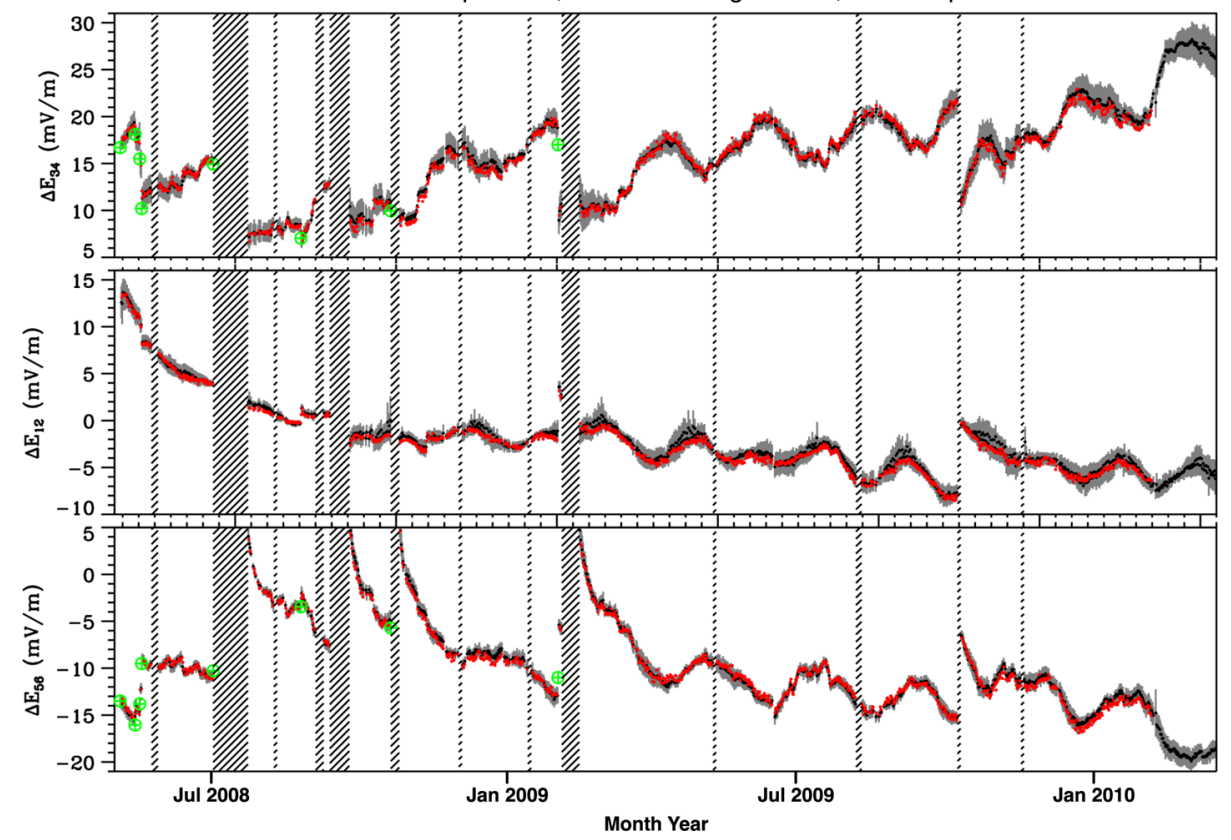

Fig. 51 Electric field offsets for the three orthogonal double probe measurements for the first two years of the C/NOFS mission deduced from different techniques, as explained in the text. Shaded regions correspond to regions when no data are available

Another approach to determine the offsets in a routine manner is to assume that the measurements, during quiet times when no spread-F is occurring, are entirely due to $\mathbf{V} \times \mathbf{B}$. Here, we take advantage of the fact that the ambient electric fields change diurnally and essentially the contributions from the ambient fields cancel when averaged over an orbit. In fact, this assumption is particularly valid for the zonal ambient electric fields, which is essentially the same approach as utilizing the $\nabla \times \mathbf{E}_{\text {zonal }}=0$ assumption. Although this is not strictly valid for the $\mathbf{E}_{\text {meridional }}$ electric fields, those ambient fields do change direction diurnally and for the most part, average close to 0.0 over an orbit. The results of the offset determination using this approach are shown in Fig. 51. We note that the $\mathbf{V} \times \mathbf{B}$ approach was the same technique used to determine the DC electric field offsets on the polar-orbiting Dynamics Explorer-2 satellite. For that satellite, offsets were determined from $\mathbf{V} \times \mathbf{B}$ measurements at low and mid-latitudes (e.g., Maynard et al. 1988) and subsequently used at high latitudes as well (e.g., Maynard et al. 1982).

Still another approach for which considerable effort was expended was to treat the offsets and the non-orthogonality unknowns as one combined system problem. To this end, as discussed in Maynard (1998), an analysis of 39 equations and 39 unknowns was performed in which the precise sphere location and the electric field offsets were treated as unknowns. Using the $\mathbf{E} \cdot \mathbf{B}=0$ assumption and utilizing data gathered over varying lengths of time, attempts were made to determine the most statistically reasonable offsets and sphere positions that supported the observations. This approach did not yield definitive conclusions, primarily because the different variables changed only slightly over the course of the analysis intervals. 

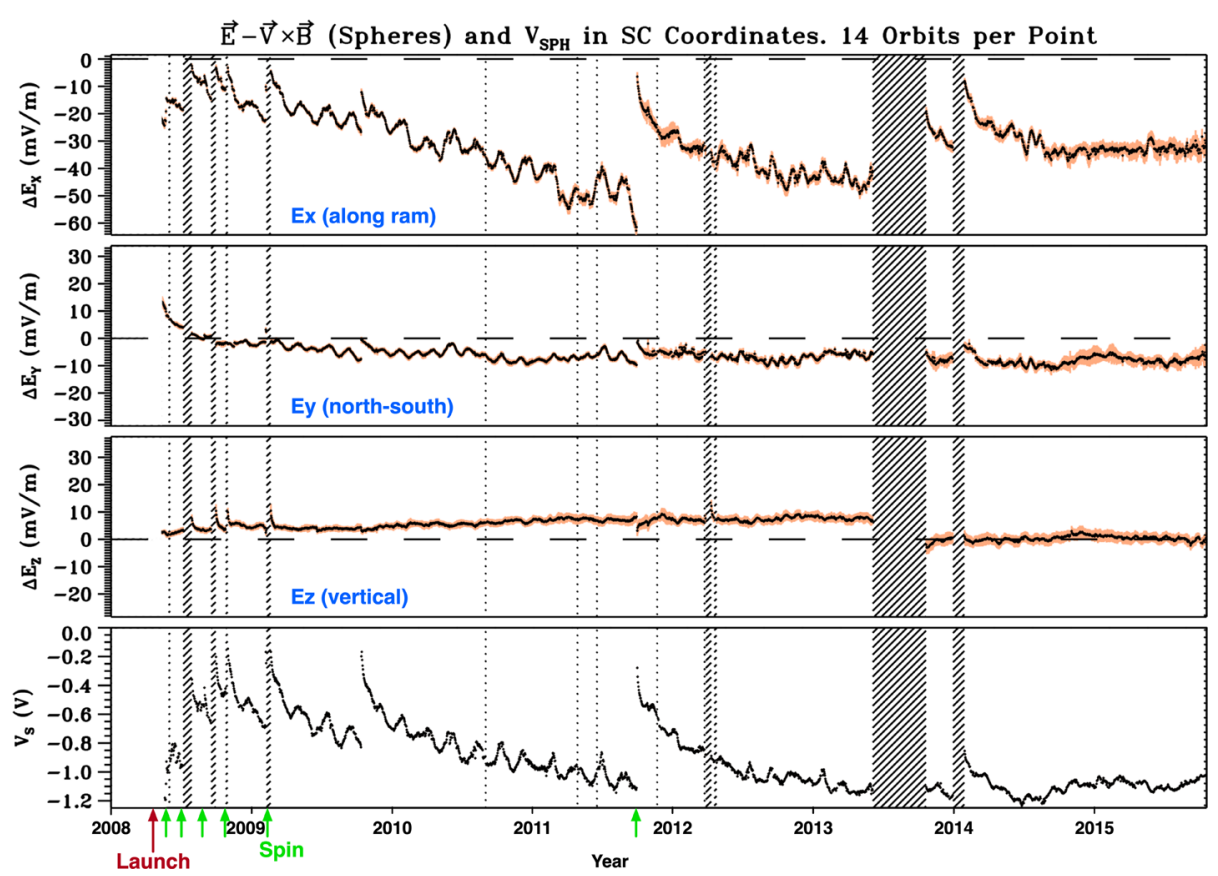

Fig. 52 DC electric field offsets rotated into spacecraft coordinates for the entire C/NOFS mission, one data point per day (14 orbits). Shaded regions correspond to times when no data are available. The orange traces correspond to the standard deviation of each offset determination

Finally, the offsets derived for the double probes in the orbit plane (essentially the E34 and E56 measurements) were independently verified using occasional spins of the C/NOFS spacecraft. These are also shown in Fig. 51 and show good agreement with the offsets determined by the other techniques.

Despite the good agreement of the offsets determined by the different techniques, Fig. 51 shows a number of noteworthy features. First, the offsets are on the order of 10 's of $\mathrm{mV} / \mathrm{m}$ and display superimposed, small variations of several $\mathrm{mV} / \mathrm{m}$ in all of the components, with periodicities of approximately 64 days that correspond to the apsidal precessional period of the satellite. Thus, as the local times of apogee and perigee changed with this time period, the probes encountered different ambient plasma conditions that subsequently produced small variations in the offsets.

A surprising result are the "jumps" in the offsets, particularly associated with offsets observed after the satellite was spun. Although such jumps did not affect the analysis of the DC electric fields, they presented a puzzle to the VEFI team. We now rotate the offsets into spacecraft coordinates and display the results for the entire mission in Fig. 52. Note the jumps in the offsets appear most pronounced in the ram (x) component, as do the large variations associated with the orbital precession. This result suggests there was a frictional charging effect along the spacecraft velocity. We interpret this as due to the presence of Torlon insulating material that was used to suspend the spheres at the ends of the booms, as discussed by Ferguson et al. (2013). Importantly, we note that such large "jumps" were not observed when offsets of the double probes comprised of the inner, "cylinder" sensors were examined. Further details are discussed in Pfaff et al. (2021). 
Fig. 53 Triaxial double probe electric field measurements with the $\mathbf{V} \times \mathbf{B}$ computed fields shown in red (upper three panels). The lower two data panels show the DC electric fields after the $\mathbf{V} \times \mathbf{B}$ and offsets were removed and the results rotated into geomagnetic coordinates

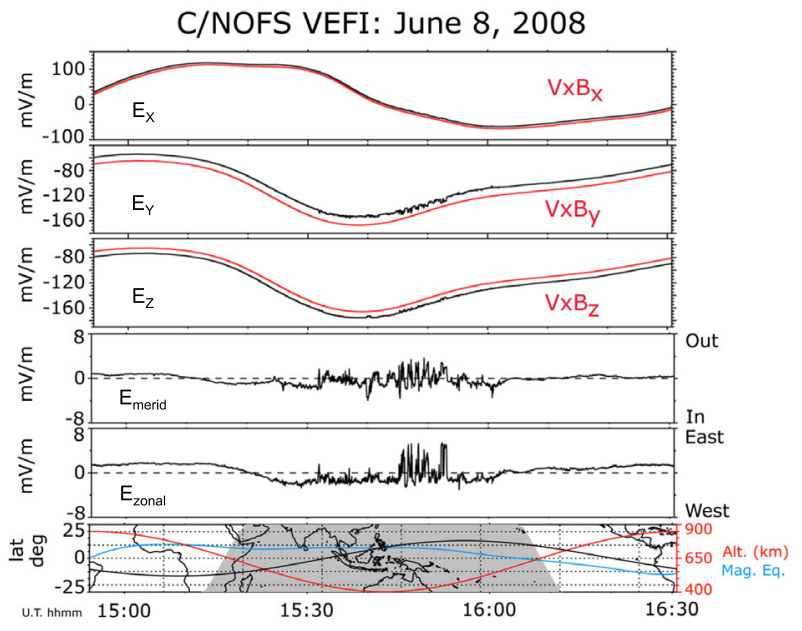

With the routine determination of reliable DC electric field offsets, the analysis of the measurements proceeded for the entire data set. An example of one orbit of data is shown in Fig. 53 in which the raw data corresponding to the three components are shown in the upper panels along with the $\mathbf{V} \times \mathbf{B}$ computed fields for each of these components. The two panels below these show the resulting DC electric fields after the $\mathbf{V} \times \mathbf{B}$ and offsets were removed and the resulting ambient electric fields were rotated into geomagnetic coordinates. The lowest panel shows the geographic footprint of the satellite along with the magnetic equator for reference and the satellite altitude. The data display is centered on midnight local time. The grey shading depicts the nighttime conditions on the Earth's surface.

\subsubsection{Electric Field Examples}

A full day (14 orbits) of electric field data is shown in Fig. 54 in which the $16 \mathrm{~s} / \mathrm{sec}$ electric field data have been rotated into magnetic (zonal and meridional) components. Here, the large scale zonal and meridional components clearly vary periodically with local time within each orbit. Note that electric field variations are superimposed on the DC electric fields and are, in fact, part of the DC electric field solution. These higher time resolution structures are pronounced during the nighttime, as shown by the eclipse intervals in the lower bar, on almost every orbit, even when no spread-F depletions are present, and are typically larger than the ambient, slowly varying DC electric fields.

Electric field measurements, averaged to $1 \mathrm{~s} / \mathrm{sec}$, are shown for two orbits in Figs. 55(a, b) where the time-series presentation is centered on local midnight. The second and third panels from the top show the meridional and zonal components of the DC electric fields, whereas the uppermost panel shows "arrow" plots of their associated $\mathbf{E} \times \mathbf{B}$ velocities. In this presentation, these drift vectors (top panel) have been averaged in time by a factor of 20 both for clarity in the arrow-type display and to emphasize the larger scale variations. The lowest data panels show the plasma density. The density data in Fig. 55(a) shows significant density depletions with enhanced zonal electric fields, corresponding to upward plasma drifts within the depletions. The two orbits shown in Fig. 55(a, b) were chosen as representative of data when perigee was located near midnight (a) and near noon (b). These data are published in Pfaff et al. (2010a). 
C/NOFS VEFI Electric Fields (16 s/sec) - May 232008

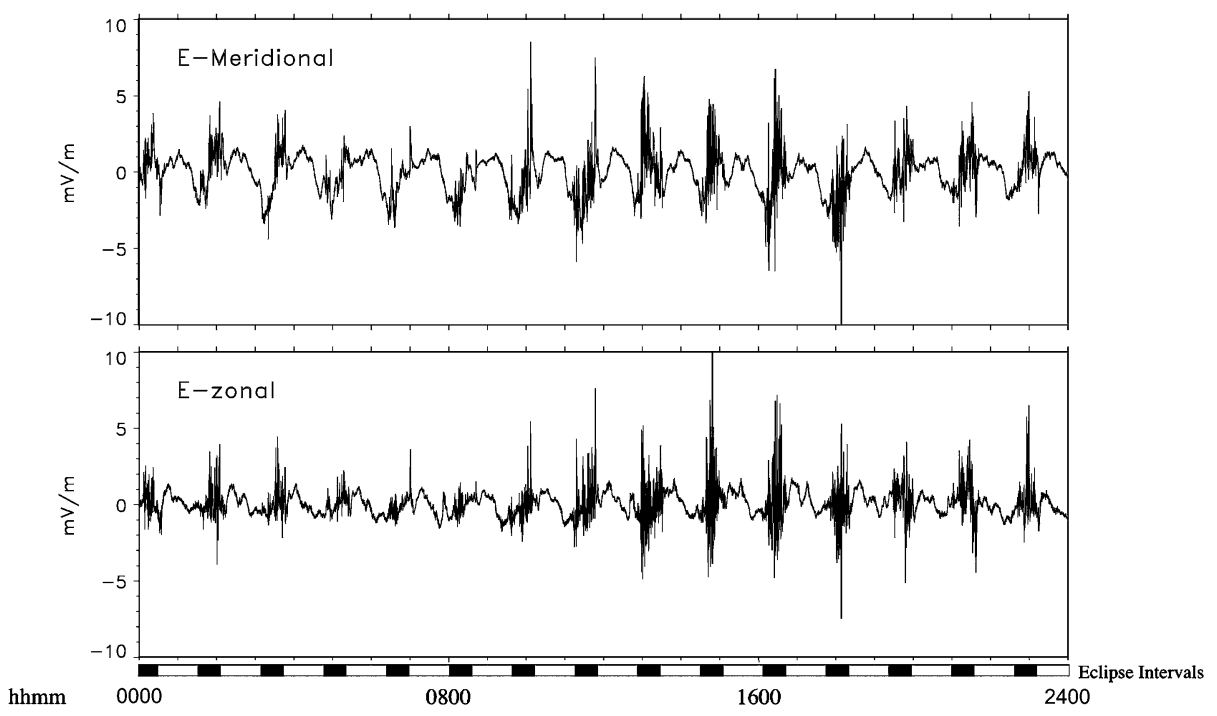

Fig. 54 A full day (14 orbits) of $16 \mathrm{~s} / \mathrm{sec}$ electric field data in geomagnetic coordinates showing the diurnally varying electric fields with superimposed, larger amplitude, structured, high time resolution electric fields that are prevalent at night

\subsubsection{Comparisons with the lon Velocity Meter and with Jicamarca Radar Observations}

We now present comparisons of the electric field data with other measurements. Figure 56 shows comparisons of $\mathbf{E} \times \mathbf{B}$ meridional plasma drifts measured by VEFI and simultaneous measurements of the same component gathered by the C/NOFS ion velocity meter (IVM) (see Heelis and Hanson 1998) for portions of two night-time passes near perigee. Note the excellent agreement between the two techniques for this component. Additional comparisons of C/NOFS drifts measured by these two techniques are published by Klenzing et al. (2011). These comparisons are in agreement with comparisons of ion drift and $\mathbf{E} \times \mathbf{B}$ drifts measured on the DE-2 satellite as reported by Hanson et al. (1993).

A comprehensive calibration/validation program for the C/NOFS data, in particular the plasma drift and plasma density measurements, was enacted by the Air Force that primarily entailed comparisons with simultaneous measurements with the Jicamarca Radar Observatory (JRO) in Peru. An example of four days of C/NOFS meridional $\mathbf{E} \times \mathbf{B}$ drifts and Jicamarca vertical velocity observations, gathered primarily in the daytime, are shown in Fig. 57. There is generally good agreement between the two techniques.

Another example of coincident JRO and VEFI vertical plasma drifts was gathered during a nighttime spread-F event reported by Hysell et al. (2009) and shown in Fig. 58. Here, detailed Doppler velocity measurements by Jicamarca within the scattering volume of the unstable ionosphere show a generally good correspondence between the radar measurements and the $\mathbf{E} \times \mathbf{B}$ vertical velocity measurements gathered on the C/NOFS satellite as it pierced the unstable ionosphere illuminated by the radar at $424 \mathrm{~km}$ altitude.

Finally, average Jicamarca zonal drifts compare well to average zonal VEFI $\mathbf{E} \times \mathbf{B}$ drifts gathered within the same longitude region for the first three years of the C/NOFS mission 
C/NOFS Orbit 756 -- June 06, 2008 (Day 158)

VEFI and PLP Observations

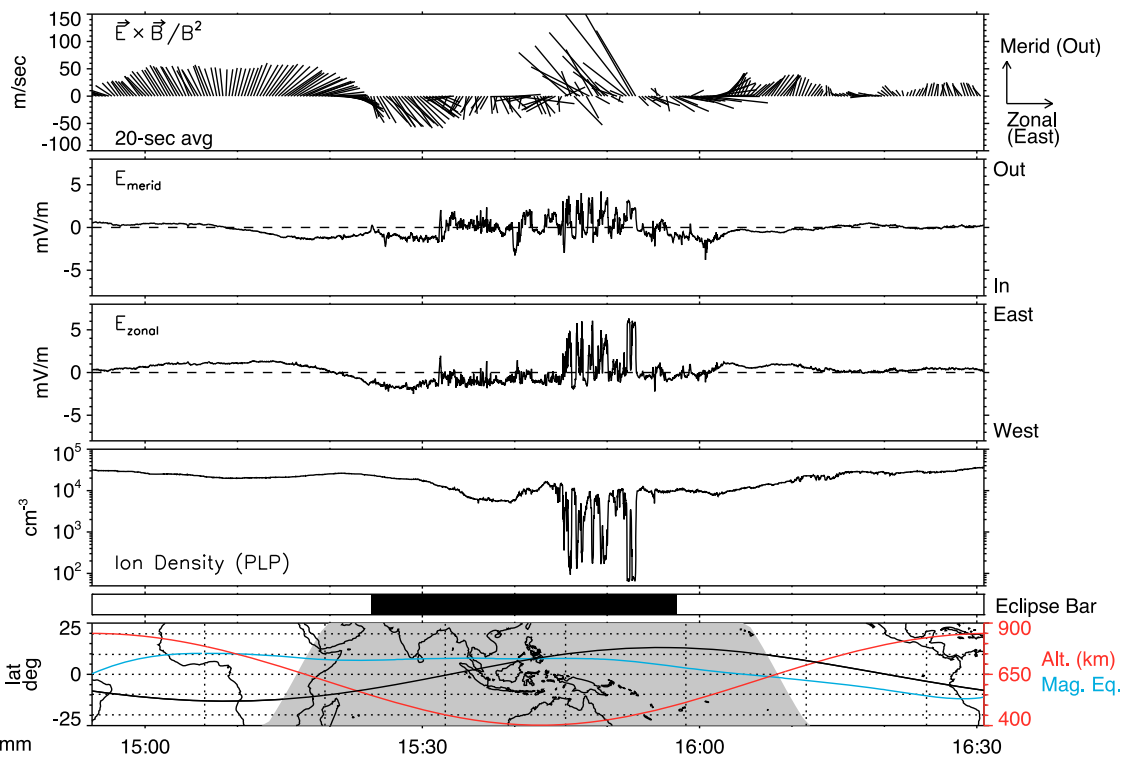

(a)

C/NOFS Orbit 387 -- May 12, 2008 (Day 133)

VEFI and PLP Observations

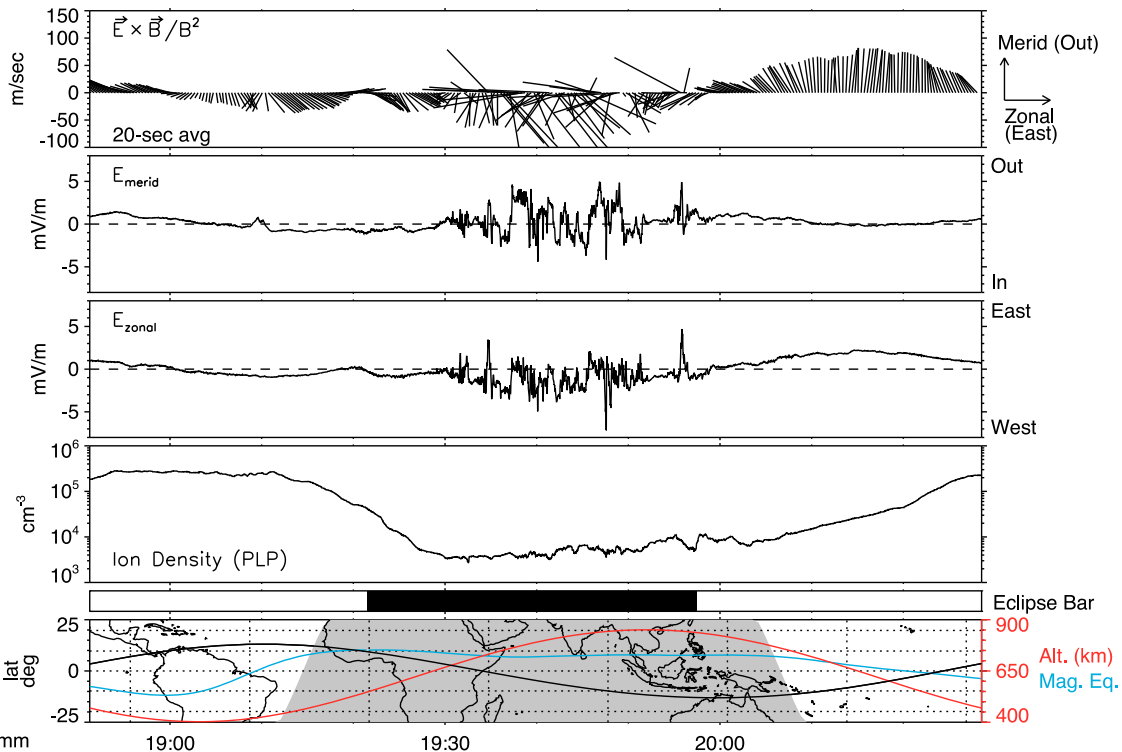

(b)

Fig. 55 Examples of vector electric fields, associated $\mathbf{E} \times \mathbf{B}$ plasma drifts, and plasma number density gathered on two orbits of the C/NOFS satellite 


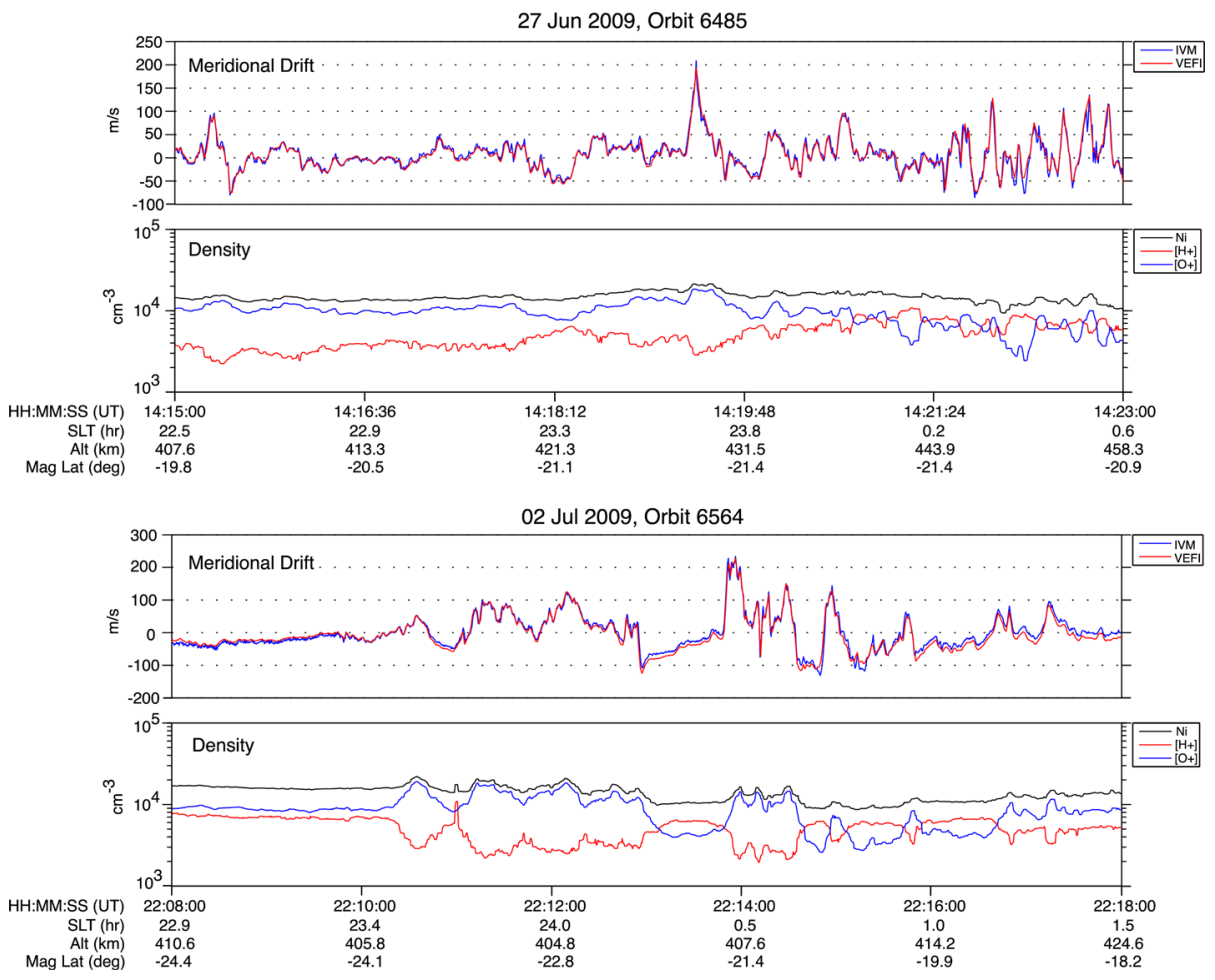

Fig. 56 Two examples of simultaneous meridional plasma drifts measured by VEFI and the ion drift meter. (Upper panels) Meridional $\mathbf{E} \times \mathbf{B}$ plasma drifts measured by the VEFI electric field instrument overlaid with the meridional ion drifts measured by the ion velocity meter for two orbits of the C/NOFS satellite. (Lower panels) Plasma density (black) measured by the Retarding Potential Analyzer in which the percentage of $\mathrm{O}^{+}$ (blue) and $\mathrm{H}^{+}$(red) ions present are also shown

as a function of season. These comparative zonal drifts have been analyzed by Fejer et al. (2013) and are shown in Fig. 59.

\subsubsection{Summary of the VEFI DC Electric Fields}

As shown above, the VEFI electric field instrument performed well throughout the C/NOFS mission and met the performance specified by the project. There were two aspects of the measurement which were surprising that are discussed in more detail in Pfaff et al. (2021): (1) The zonal component of the DC electric field exhibited a bias that varied systematically with the apsidal precession period ( $\sim 2$ months) which was subsequently removed with a detrending algorithm. This bias may have resulted from charging associated with the torlon insulator that held the spherical sensors at the tips of the booms, as discussed above with respect to the observed variations of the zonal electric field offset. This effect can be easily removed on future missions by including symmetric guards in-board and out-board of the sphere driven at the output of the pre-amp. The requirement to cage the spheres at their base when stowed did not enable this option to be utilized for the boom systems that were provided for use in this experiment. (2) Spurious fields were sometimes observed which may have resulted from capacitance/inductance loading of the pre-amplifiers during certain 

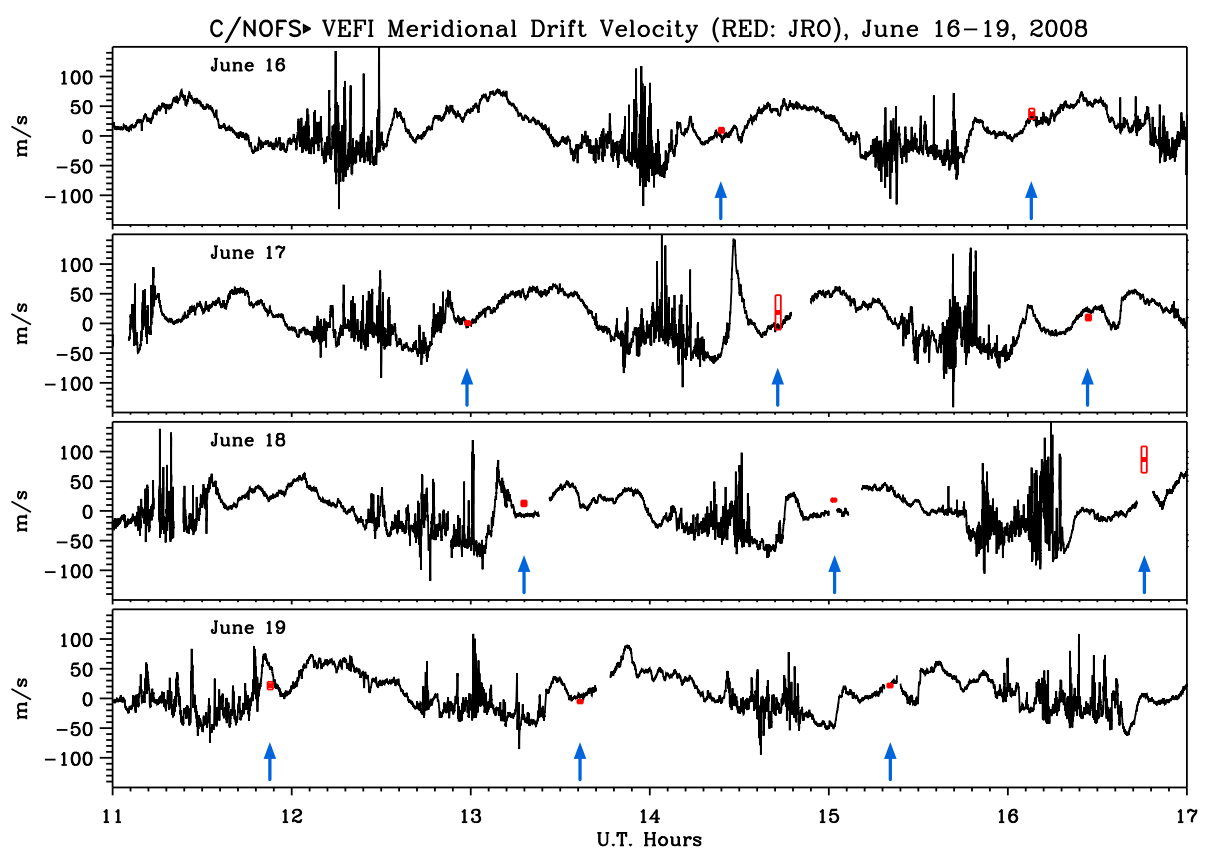

Fig. 57 Meridional $\mathbf{E} \times \mathbf{B}$ plasma drifts measured by the VEFI electric field instrument for 6 hours of four consecutive days in June, 2008, in which simultaneous measurements of the vertical drift were measured by the Jicamarca incoherent scatter radar. The radar velocities are shown in red for the times when the C/NOFS satellite orbit traversed the same longitude. The blue arrows simply guide the eye to the conjunctions

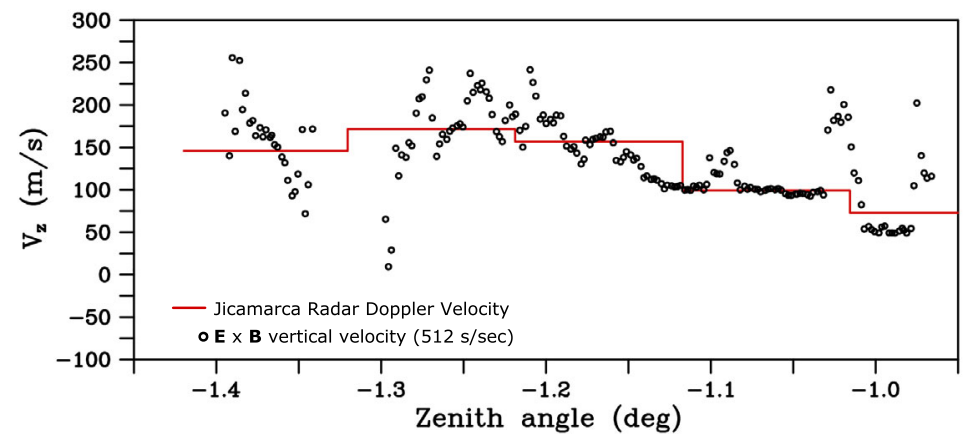

Fig. 58 Meridional $\mathbf{E} \times \mathbf{B}$ plasma drifts measured by the VEFI electric field instrument at $512 \mathrm{~s} / \mathrm{sec}$ for a brief interval in which the satellite flew through the vertical beam of the Jicamarca radar at $424 \mathrm{~km}$ altitude during an active spread-F irregularity event. The simultaneous radar-measured velocities are shown in red. See Hysell et al. (2009) for details

plasma conditions. Such effects had not been observed previously on electric field experiments on ionospheric missions and can be eliminated in a straightforward manner for future experiments. 
Fig. 59 Comparison of average zonal $\mathbf{E} \times \mathbf{B}$ plasma drifts measured by VEFI in the western American longitude sector and the Jicamarca radar-measured $\mathrm{F}$ region zonal drifts (from Fejer et al. 2013)

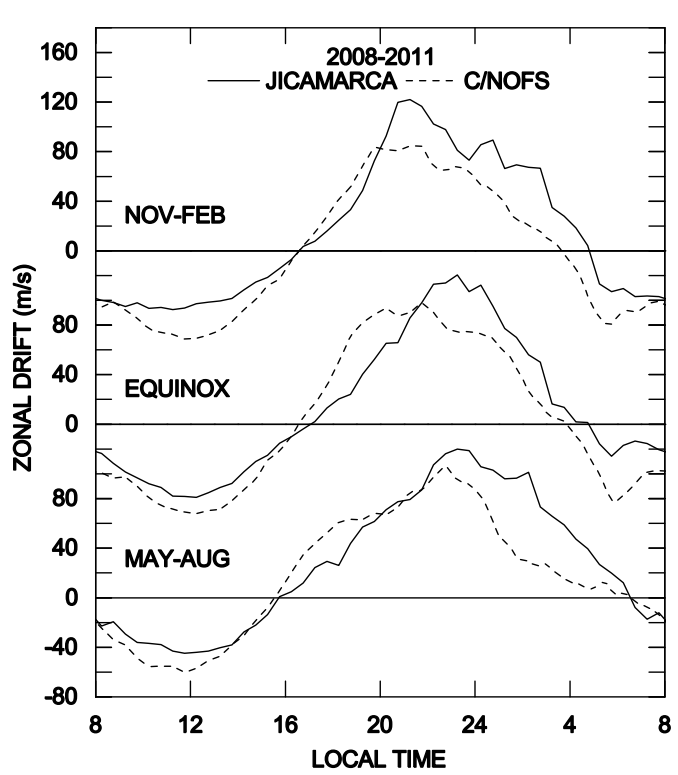

\subsection{AC Electric Fields and Examples}

In addition to the DC electric field data, VEFI also produced $\mathrm{AC}$ electric field measurements in different frequency regimes. These data were recorded by the instrument as outlined in the previous sections. Here we show examples of data collected in the different frequency regimes to illustrate the instrument performance and high quality of the measurements.

Continuous measurements of two orthogonal ELF electric field channels are shown in the spectrograms in Fig. 60 for two complete orbits. The lowest panel shows the geographic position of the C/NOFS satellite along its orbit and its altitude. The magnetic equator is provided as a reference and the black bar shows when the satellite was in eclipse. The data illustrate the preponderance of ELF hiss in the low latitude ionosphere, for which the sharp cut-offs in frequency depend on the ambient magnetic field and ambient thermal ions present (e.g., Chen et al. 2020). The lowest frequencies $(<50 \mathrm{~Hz})$ show evidence of Schumann resonances first observed in space by the C/NOFS VEFI instrument and reported by Simões et al. (2011). Because two components of the AC ELF electric field were gathered simultaneously in the orbit plane, cross spectra between these two components readily distinguish circular from linear polarization of the wave modes as well as provide information regarding wave cutoffs and absorption features of the electromagnetic components.

An example of the broadband electric field wave data is shown in Fig. 61. Here, spectrograms from two orthogonal electric field channels, each sampled at $2048 \mathrm{~s} / \mathrm{sec}$, are shown, extending the spectral measurements to $1024 \mathrm{~Hz}$. The uppermost panel displays the output of the filter bank data for this interval.

We turn now to the on-board FFT data. Figure 62 shows a series of subsequent orbits, with time increasing vertically down the page. Here are shown daytime VLF spectrograms produced from 128 spectral bins every 2 seconds from the on-board FFT processor. The right-hand panel show the coincident Dst measurements, showing the appearance of a strong magnetic storm during which these measurements were gathered. 


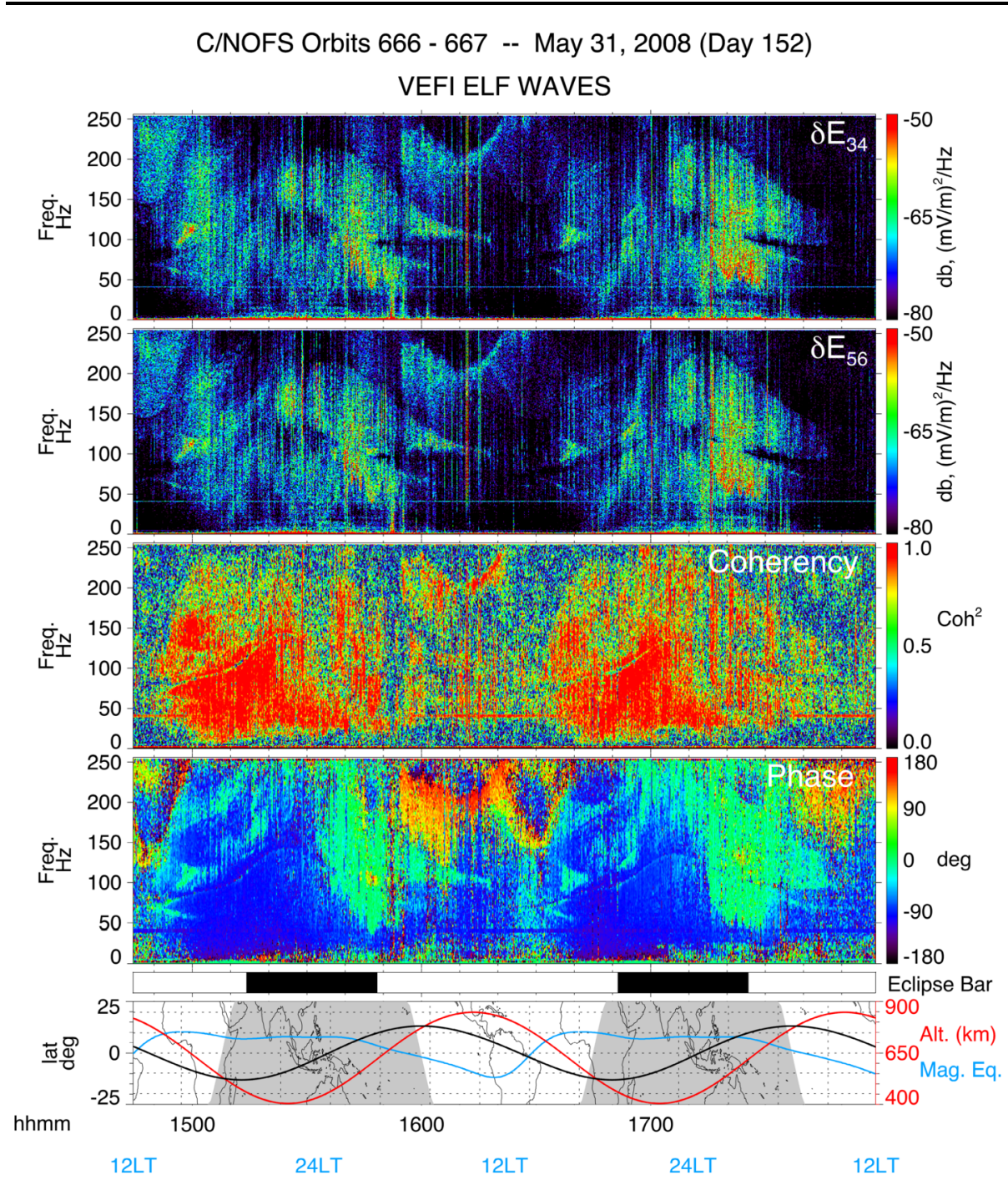

Fig. 60 Spectrograms, computed on the ground, of the time series electric field wave data from the orthogonal "3-4" and "5-6" double probes sampled at $512 \mathrm{~s} / \mathrm{sec}$ for two consecutive orbits. The phase and coherency computed from the cross spectra of these two channels are also shown. The eclipse bar shows when the satellite was in darkness and the lowest panel shows the satellite location and altitude

In Fig. 63, we display an example of the HF FFT data along with simultaneous measurements from the on-board VLF FFT data. Both data panels show waves with definitive lower cutoffs. As discussed in Pfaff et al. (2010b), the lower frequency cutoff displayed in the upper panel (HF data) corresponds to the Z-mode cutoff which depends on the plasma frequency, and hence provides an independent measurement of the ambient plasma density, shown in the lowest data panel. The lower frequency cutoff shown in the VLF spectrogram (below the HF spectrogram) corresponds to the local lower hybrid frequency, which depends on both the ambient plasma density and the local ion composition, notably $\mathrm{O}^{+}$and $\mathrm{H}^{+}$. 


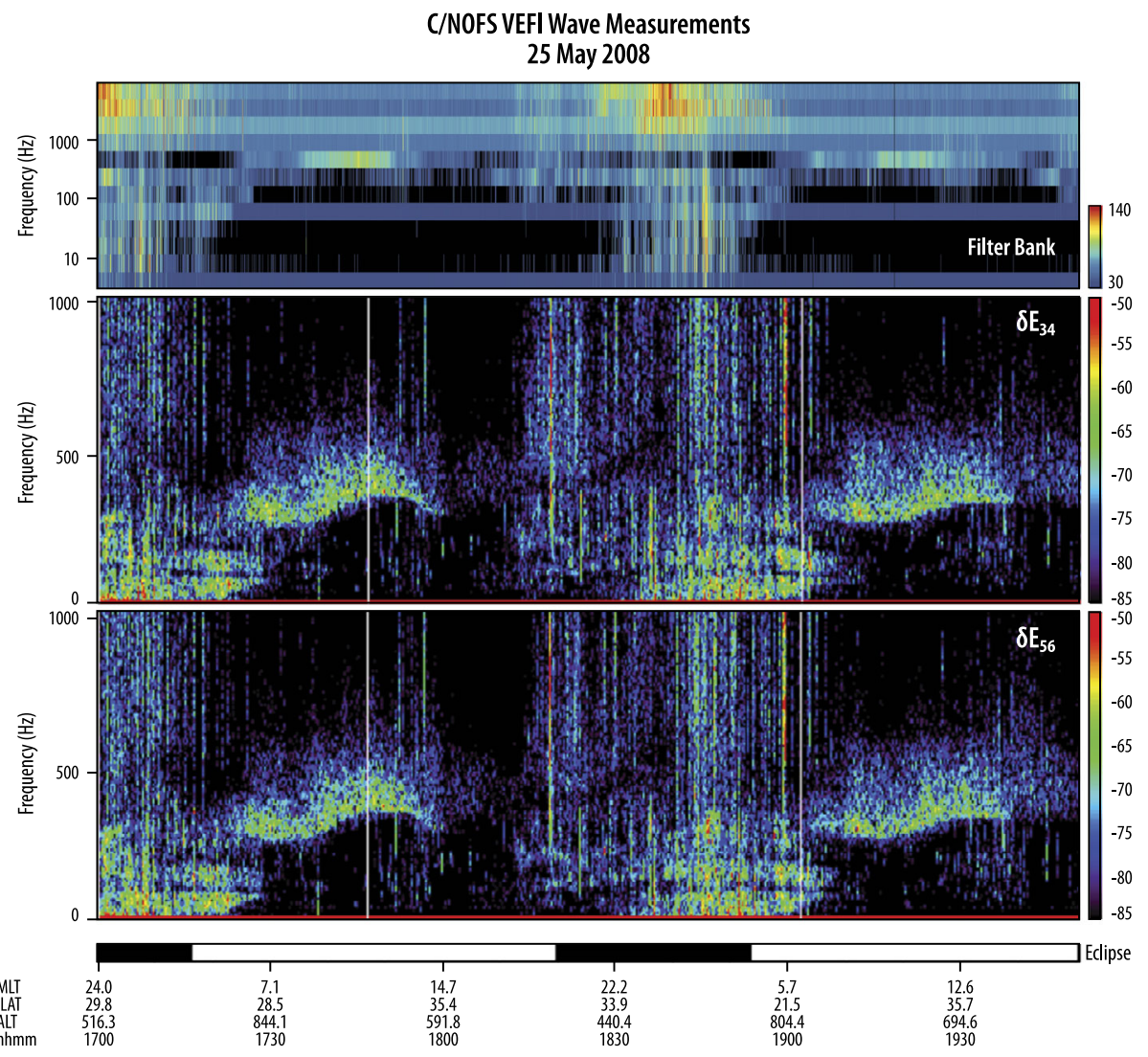

Fig. 61 (Top panel) Filter bank data from 12 logarithmically spaced frequency channels from $3 \mathrm{~Hz}$ to $8192 \mathrm{~Hz}$. (Middle and lower panels) Spectrograms, computed on the ground, of the time series electric field wave data from the "3-4" and "5-6" double probes sampled at $2048 \mathrm{~s} / \mathrm{sec}$ for approximately two orbits

\subsection{Magnetic Fields}

The three components of the flux-gate magnetometer's magnetic field time series data are processed in a manner that is initially similar to the electric field time series data processing. The quality of the measurements are inspected and interference due to torque rods and other non-geophysical effects are identified and removed. The data are converted to nT using the nominal (pre-flight) gains and calibrations. Matrices correcting for non-orthogonality are applied and the data are rotated to geographic coordinates. The model magnetic field is subtracted and the resulting difference or "delta B" fields in each direction are retained for detailed scientific analysis.

An example of one day (14 orbits) of magnetic field data is shown in Fig. 64. Note the rhythmic variations of the magnetic field as the satellite changes longitude and altitude along each orbit. Similar to the DC electric field data that is dominated by $\mathbf{V} \times \mathbf{B}$, these large-scale, periodic variations provide an immediate indication of the offsets of the three components. The data also reveal magnetic contributions from torque rod firings with durations that varied from 5-10 seconds early in the mission to over a minute later in the mission. With typical amplitudes of 100-1000 nT (depending on the component), these intervals are 


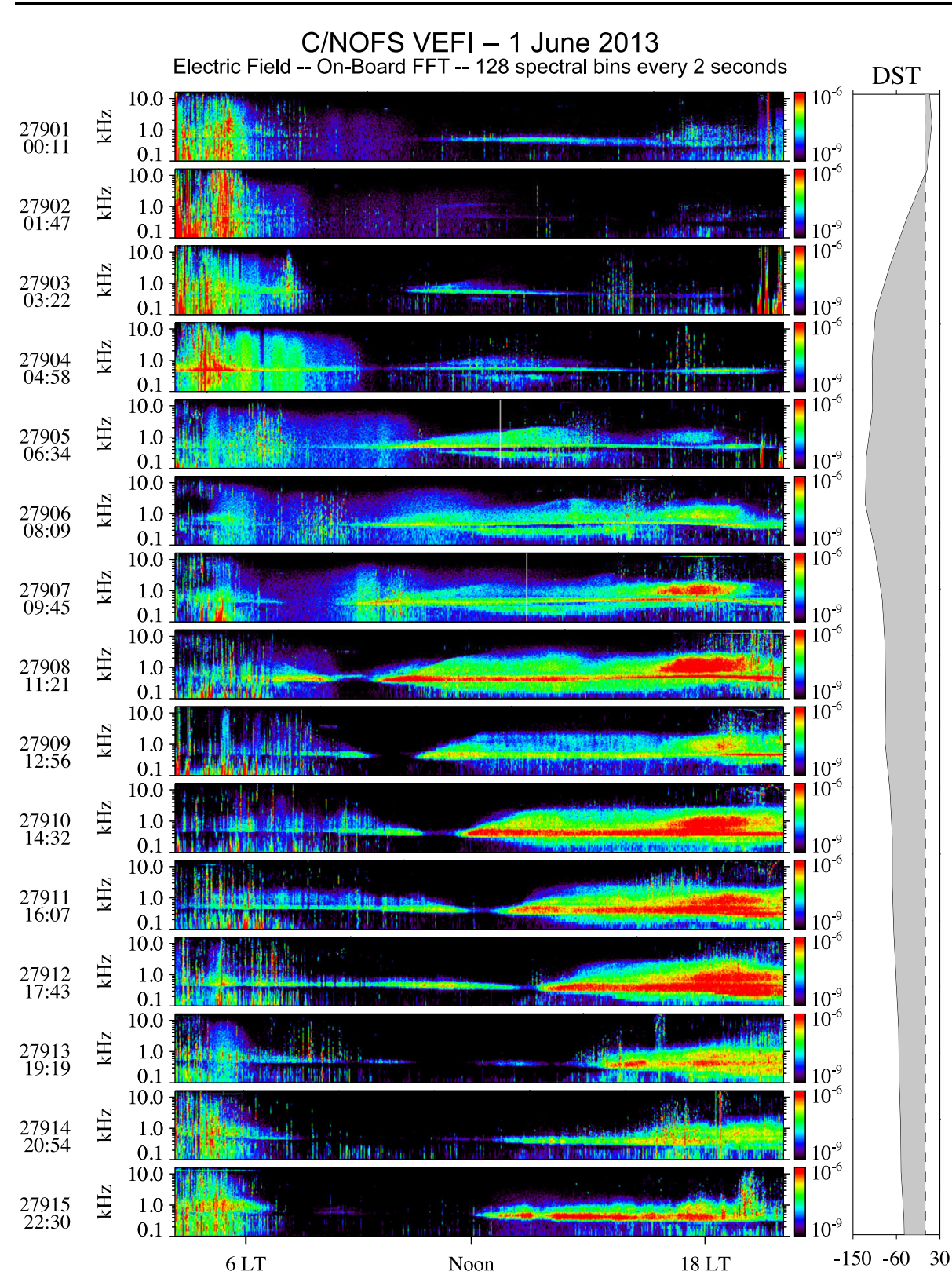

Fig. 62 VLF spectrograms computed on board the satellite of the "3-4" electric field double probe data for 15 consecutive orbits on June 1, 2013 centered on the daytime. The data were gathered during a magnetic storm, as shown by the Dst data plotted in the panel on the right-hand side

easily identified in the data and are removed. An option in the magnetometer processing software enables these intervals to be replaced with interpolated magnetic field data with an appropriate flag set to indicate the intervals when this replacement is inserted. 


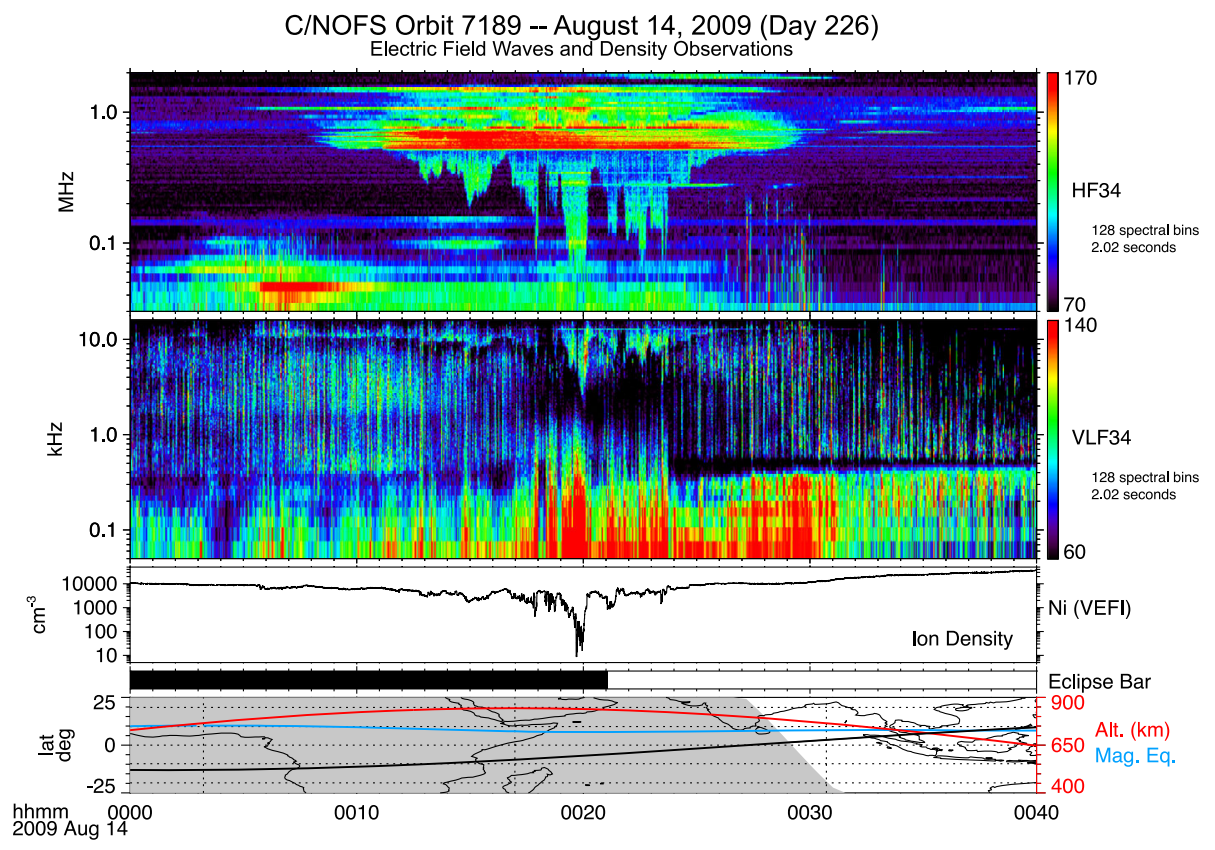

Fig. 63 HF (upper) and VLF (lower) spectrograms computed on board the satellite of the "3-4" electric field double probe data for a 40-minute period as the satellite traversed the African-Asian longitudes. The third panel from the top shows the plasma density, below which is the eclipse bar and map showing the satellite location and altitude. The lower cut off of the natural HF and VLF emissions depends on the plasma density. The HF spectrogram shows strong ground transmitters detected between $500-800 \mathrm{kHz}$

Figure 65 shows the same data as in Fig. 64, but organized such that all 15 orbits are superimposed and plotted as a function of longitude (top) and local time (bottom). Here, magnetic field model data shown in red are superimposed on the measurements shown in black. The measurements are very well represented by the magnetic field model which, in this presentation, mask the actual observations, except for the presence of torque rod firings which are easily identified as black excursions. Note that the torque rod firings are broadly distributed in both longitude and local time.

A key aspect of the magnetic field analysis is the identification of the offsets of the three components. Figure 66 show the offsets derived from the measurements for the entire $\mathrm{C} / \mathrm{NOFS}$ mission. The offsets are shown in black for the three components in the upper three panels. Note they vary slowly on the order of 10's of nT. We also show, in the lowest panel, the temperature data from the magnetometer sensor on the boom. The offsets, particularly for the $\mathrm{x}$ and $\mathrm{y}$ components, "track" the temperature. In fact, we have identified a linear relationship between temperature and the difference between measurement and model. This relation allows us to rectify the magnetometer data to a fixed temperature. Details may be found in Freudenreich and Pfaff (2021).

The VEFI magnetometer measurements compare very well to the magnetic field models (e.g., POMME, CHAOS, IGRF). Daily offsets (14 orbits/day) were calculated using the average of magnetometer measurements minus a model. This was carried out for the IGRF, POMME, and CHAOS models with little significant difference between the resulting offsets. The magnetometer gains and orthogonality matrix were also calculated based on the models. The standard deviation of data taken over several orbits is highly sensitive to non- 


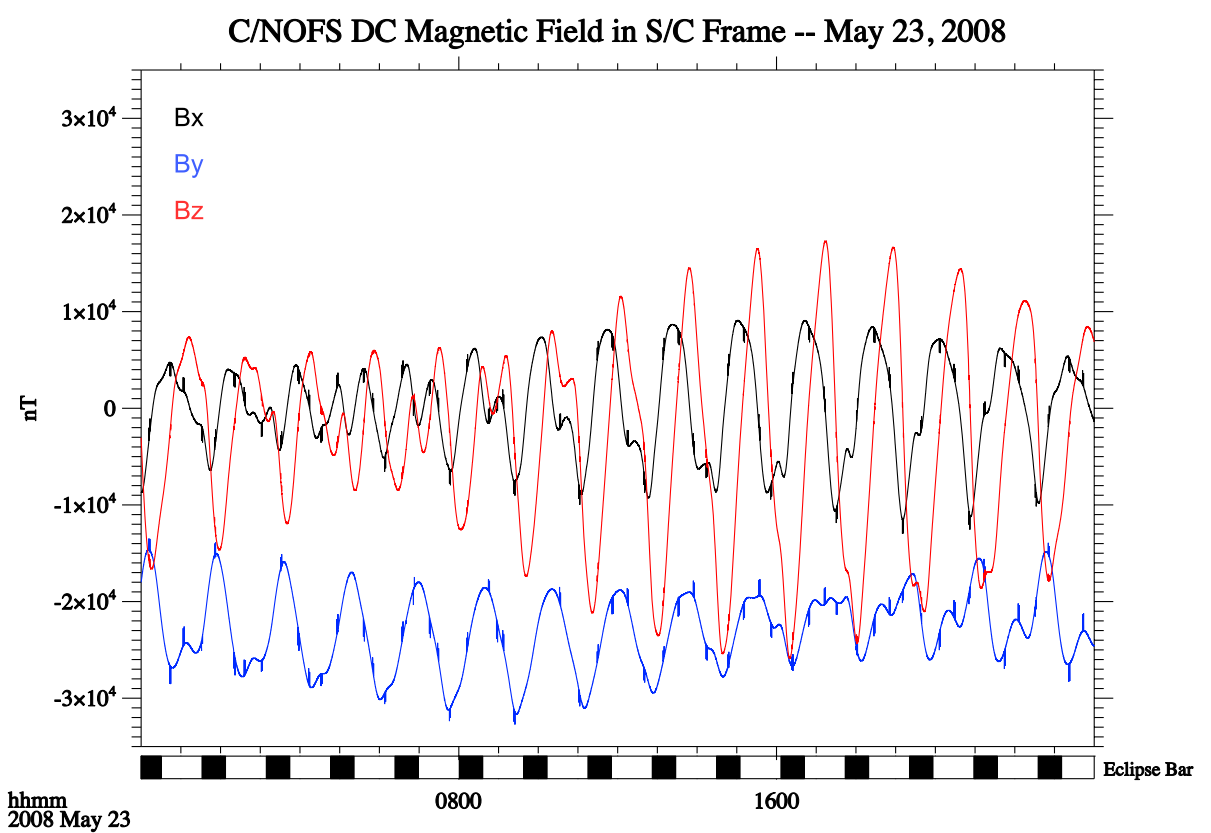

Fig. 64 Three components of "raw" DC magnetic field data for a full day of the C/NOFS mission. The lowest panel shows the times in which the spacecraft was in eclipse. Narrow spikes are superimposed on the magnetic field data which correspond to signatures of the magnetic torque rods

orthogonality. The magnetic field data, minus a modified IGRF model (see Freudenreich and Pfaff 2021, for details), is shown as a function of latitude and longitude for all three components in Fig. 67 for the first 7 years of the mission. In general, the agreement is better than $3 \mathrm{nT}$. Such relative magnetic fields are of very high quality and have been shown to be exceptionally useful for examining currents and magnetic field variations throughout the entire C/NOFS mission.

To illustrate the performance of the VEFI magnetometer, we present two examples of the DC magnetic field data gathered on the C/NOFS satellite, one that shows observed currents and one that shows time series magnetic field observations.

Figure 68 shows an example of electrojet currents observed by the C/NOFS magnetometer as a function of longitude for spring conditions. The upper two panels show eastward electrojet currents exhibiting tidal modulation in the 11-13 LT range which agree nicely with both electrojet theory and other satellite measurements of the daytime electrojet, such as those gathered on the CHAMP satellite as reported by Lühr et al. (2004). Notice that the existence of a strong zonal current is revealed not only in the field-aligned component but also in the meridional (up-down) magnetometer component. The lower two panels show evidence of westward electrojet data in the early morning hours (5-7 LT) flowing along the magnetic equator, as first reported using the C/NOFS magnetic field data (Pfaff et al. 2018).

Figure 69 shows time series data of the electric field, magnetic field, and density associated with plasma depletions in the northern (a) and southern (b) hemispheres (Pfaff et al. 2013a). The zonal and meridional magnetic field perturbations are shown in the upper two panels of each set of measurements shown. Superimposed are the simultaneous electric field perturbations. Here the $\Delta \mathrm{B}$ and $\Delta \mathrm{E}$ perturbations are clearly organized about the density depletions shown in the lowest panel. The Poynting flux $(\Delta \mathbf{E} \times \Delta \mathbf{B})$ is shown in the panel 


\section{C/NOFS Magnetometer Data - 23 May 2008 Measurements - Black, Model - Red}
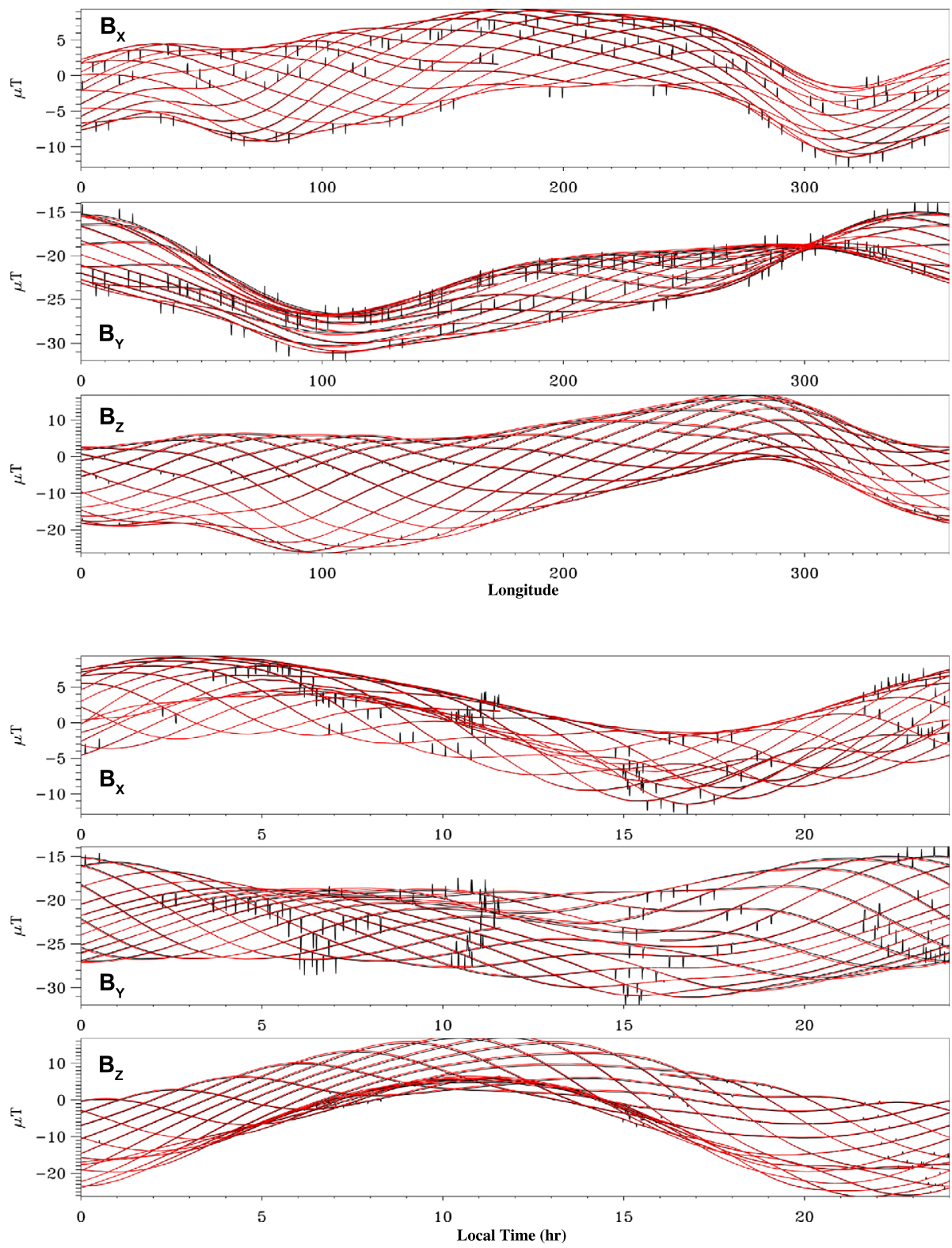

Fig. 65 The same magnetic field data as shown in Fig. 64 organized by longitude (top) and local time (bottom) with each component (Bx, By, and $\mathrm{Bz}$ ) shown in a different panel. Superimposed on the data are the corresponding data from the IGRF model. The small black spike-like signatures correspond to the magnetic torque rods 


\section{C/NOFS Magnetometer Offsets}
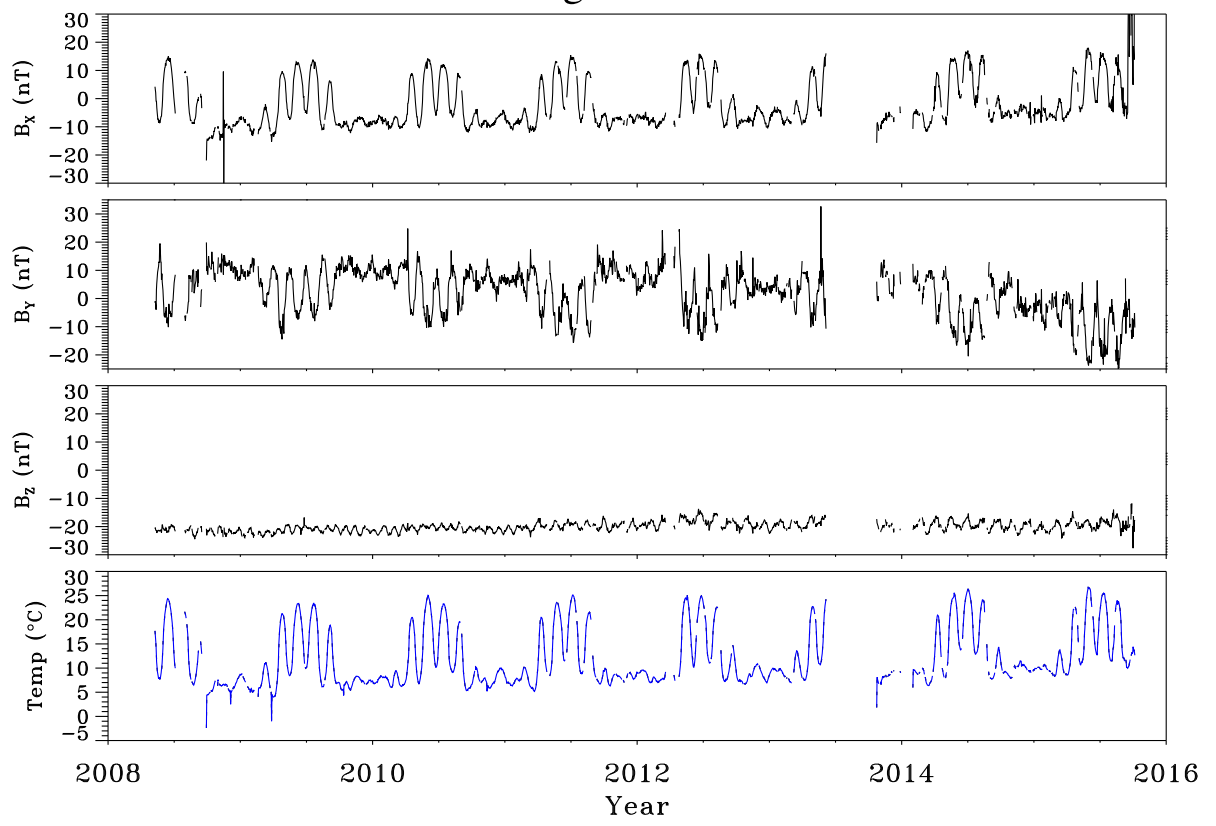

Fig. 66 Offsets computed for each of the three magnetic field components for the entire C/NOFS mission. The Bx and By components show a strong dependency on the measured temperature of the sensor, which is plotted in the lowest panel

second to the bottom. Note the Poynting flux is directed along the magnetic field direction and hence changes direction between the northern hemisphere (a) and southern hemisphere (b) data shown. Importantly, the middle panel shows the parallel magnetic field component corresponding to the magnetic fields associated with the diamagnetic cavity of the plasma depletions (see Stolle et al. 2006) and illustrates the high-quality magnetic field measurements captured by the VEFI magnetometer on the C/NOFS satellite.

Finally, we show an example of the AC-coupled magnetic field data associated with spread-F irregularities in Fig. 70. The upper two panels of this figure show enhanced magnetic field variations observed in one AC magnetic field channel, which correspond to strong plasma density variations associated with plasma irregularities near $400 \mathrm{~km}$ altitude. Such magnetic variations were frequently observed within the C/NOFS data set.

\subsection{Langmuir Probe}

The VEFI Langmuir Probe was included in the VEFI instrument suite to provide a straightforward and low cost means to gather plasma density irregularities in a fast mode and to enable density data to be readily available (i.e., within the same electronics) to identify plasma depletions associated with spread-F as an input trigger for the VEFI burst memory.

As discussed above, the probe consisted of a spherical sensor biased with a fixed negative Voltage, from which current from positive ions was collected. The bias could be varied from $-1.25,-2.5,-3.75$, and $-5 \mathrm{~V}$ to accommodate possible spacecraft charging variations and ion composition variations. For the majority of the time, the VEFI Langmuir Probe was 
C/NOFS: Magnetometer Data - Modified Model

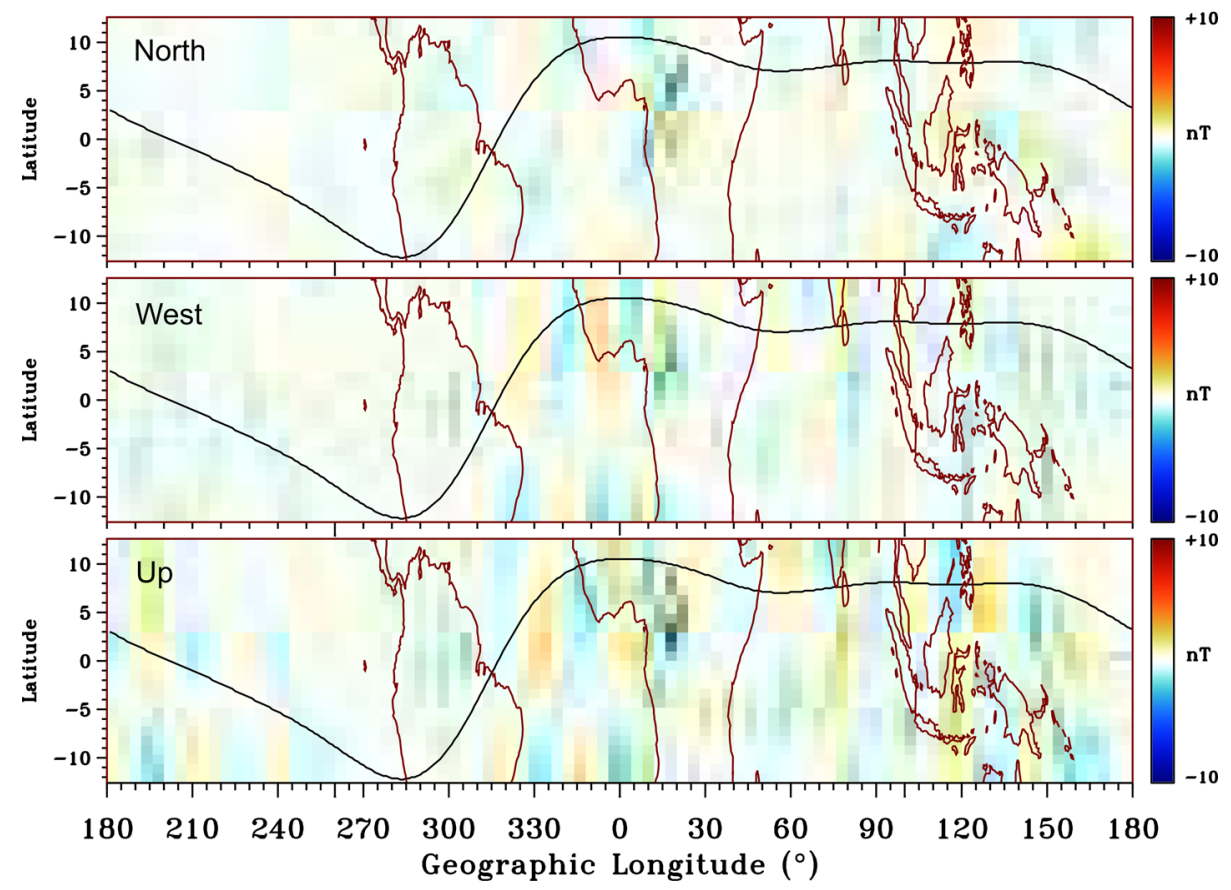

Fig. 67 Distribution of residual magnetic fields displayed in geographic coordinates for the north-south (top), east-west (middle), and up-down (bottom) components after the model field has been subtracted

biased at $-3.75 \mathrm{~V}$. Occasionally, the probe voltage was stepped in an attempt to determine the ion composition, as discussed by Brace (1998).

Although the main objective of this probe was to gather relative plasma density variations, the data can be converted to absolute density values under certain conditions. The conversion of collected ion current to plasma density from a fixed-bias probe such as the VEFI Langmuir Probe is not straightforward, since this conversion depends on the ambient ion composition as well as the ion temperature, probe bias voltage, and spacecraft potential, as discussed in detail by Klenzing and Rowland (2012). In particular, as shown by these authors, the collected current from an ambient $\mathrm{H}^{+}$plasma is larger than that from an $\mathrm{O}^{+}$ ambient plasma. Hence, knowledge of the ion composition is needed to reliably convert the collected current to plasma density.

VEFI converts the collected current to plasma density using a number of different methods:

Method 1: Use an empirical conversion based on a pre-launch calibration

Method 2: Assume the plasma is $100 \% \mathrm{O}^{+}$with a temperature of $800 \mathrm{~K}$

Method 3: Use predicted $\mathrm{O}^{+} / \mathrm{H}^{+} / \mathrm{He}^{+}$fractions and ion temperatures from the IRI model

Method 4: Use measured $\mathrm{O}^{+} / \mathrm{H}^{+} / \mathrm{He}^{+}$data and temperatures from simultaneous RPA measurements

The standard conversion procedures employed by the VEFI team utilize the $\mathrm{O}^{+} / \mathrm{H}^{+} / \mathrm{He}^{+}$ data gathered simultaneously by the RPA instrument on C/NOFS (Method 4) provided as part of the CINDI investigation by the University of Texas at Dallas (e.g., Heelis and Han- 
Fig. 68 Average magnetic field variations at 11-13 LT (upper set of data) and 5-7 LT (lower set of data) plotted in a

longitude-latitude format for the field-aligned component (generally north-south) in the upper panels of each pair and the meridional component (generally up-down) in the lower panels of each pair. The 11-13 LT data show strong evidence of the eastward equatorial electrojet current with significant variations in longitude, whereas the 5-7 LT data show observations of westward electrojet current. The magnetic equator is depicted by the solid thin black line on each panel
C/NOFS: $\triangle \mathrm{B}, 1100-1300$ hrs Local Time, Spring

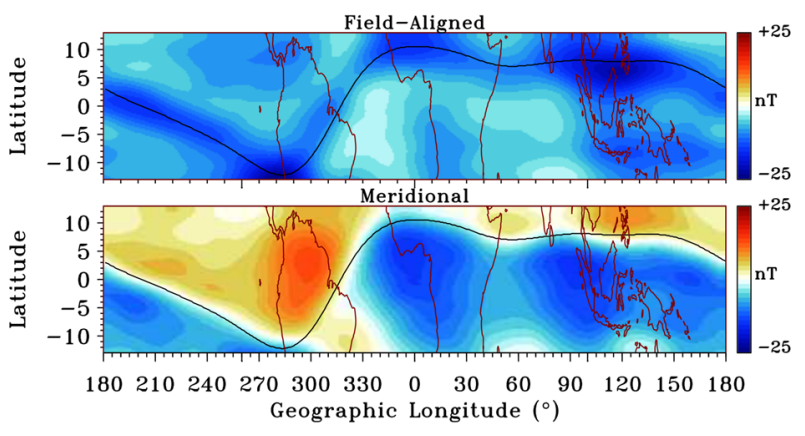

C/NOFS: $\triangle \mathrm{B}$, 0500-0700 hrs Local Time, Spring

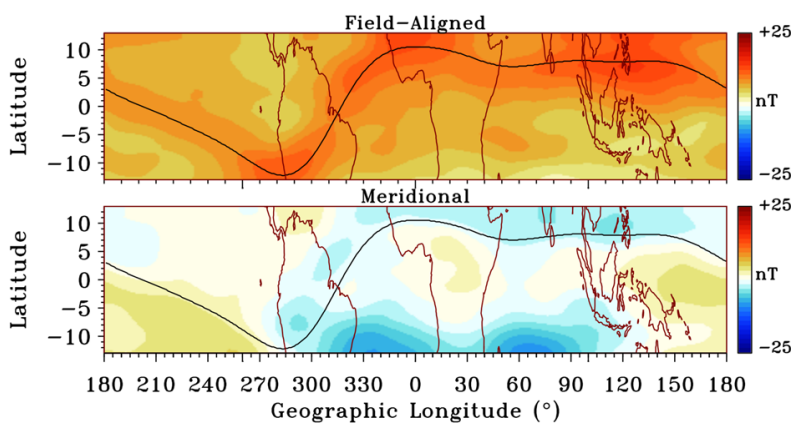

son 1998), or assumed the plasma is $100 \% \mathrm{O}^{+}$(Method 2). Figure 71 shows a C/NOFS pass in which the collected current has been converted to plasma density, assuming both an $\mathrm{O}^{+}$plasma as well as the measured $\mathrm{O}^{+} / \mathrm{H}^{+} / \mathrm{He}^{+}$data from the RPA. The absolute plasma density from the AFRL Planar Langmuir Probe (PLP) is also shown. Clearly, the inclusion of the $\mathrm{O}^{+} / \mathrm{H}^{+} / \mathrm{He}^{+}$measurements provides a density that is more consistent with the PLP absolute density measurements.

As stated above, the main purpose of including the VEFI Langmuir probe was to have a fast probe to collect high frequency density variations including $\mathrm{dN} / \mathrm{N}$ oscillations and to provide a self-contained VEFI trigger signal that could be input to the burst memory.

Two examples of observed plasma density variations are provided in Fig. 72 (a, b). In Fig. 72(a), a series of plasma depletions associated with spread-F is shown. Note that the plasma depletions extended over 4 orders of magnitude and reached values lower than $10^{2} / \mathrm{cm}^{3}$. The density variations from the same probe are also shown, in which power spectra density spectra, computed on the ground, are clearly associated with the plasma depletions. Simultaneous electric field variations show similar, coincident broadband power spectra, indicative of the electrostatic nature of the irregularities.

Plasma density data shown in Fig. 72(b) reveals a large expanse (over 3000 km horizontal distance) of strong density variations, which were observed routinely by the VEFI sensors at lower altitude $(<450 \mathrm{~km})$, and in which no large-scale plasma density structures or depletions were observed. These intense waves appear near $10 \mathrm{~Hz}$, corresponding to scale lengths of approximately $1 \mathrm{~km}$ (assuming near-stationary waves with a near-horizontal orientation) and resemble the bottomside sinusoidal oscillations reported by Valladares et al. (1983). Spectrograms of the density and electric field variations in Fig. 72(b) are illustrative 


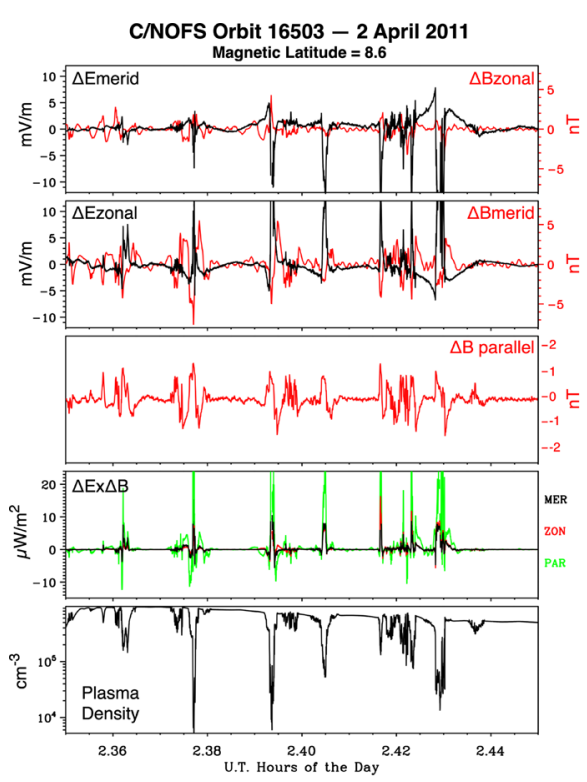

(a)

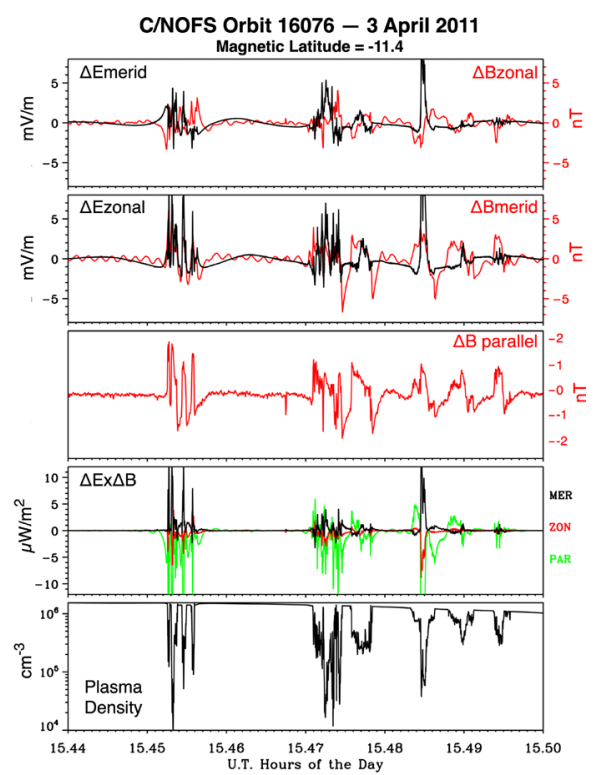

(b)

Northern Hemisphere

Southern Hemisphere
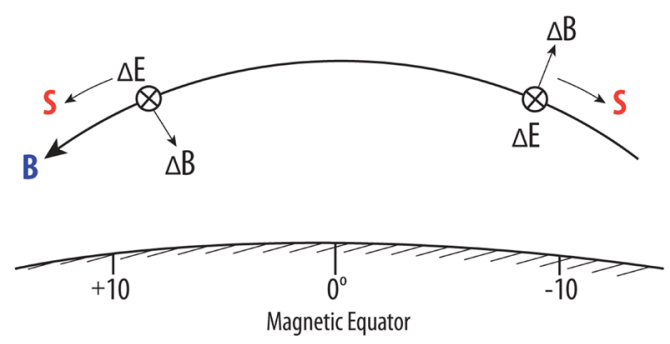

Fig. 69 Magnetic and electric field variations (upper three panels) associated with plasma density depletions (lowest panel) for portions of two nighttime passes of the C/NOFS satellite. The second-to-the-lowest panel shows the Poynting flux which is directed along the magnetic field and changes direction between the two events which correspond to the northern magnetic latitudes in (a) and southern magnetic latitudes in (b)

of how the waves appear in a narrow frequency regime near $10 \mathrm{~Hz}$, particularly near their onset. Observations such as these revealed a new source of scintillations, independent of density depletions, with important space weather implications (Pfaff et al. 2013b).

\subsection{Lightning Detector}

The VEFI Lightning Detector (LD) was designed by the University of Washington (R. Holzworth and M. McCarthy). As discussed above, two detectors were oriented north and south along the spacecraft trajectory. The digital data were provided on board as a series of counts from each detector for different amplitude levels. In addition, the "raw" photometer output from each channel was available for digitization using the VEFI burst memory A/D converters. 


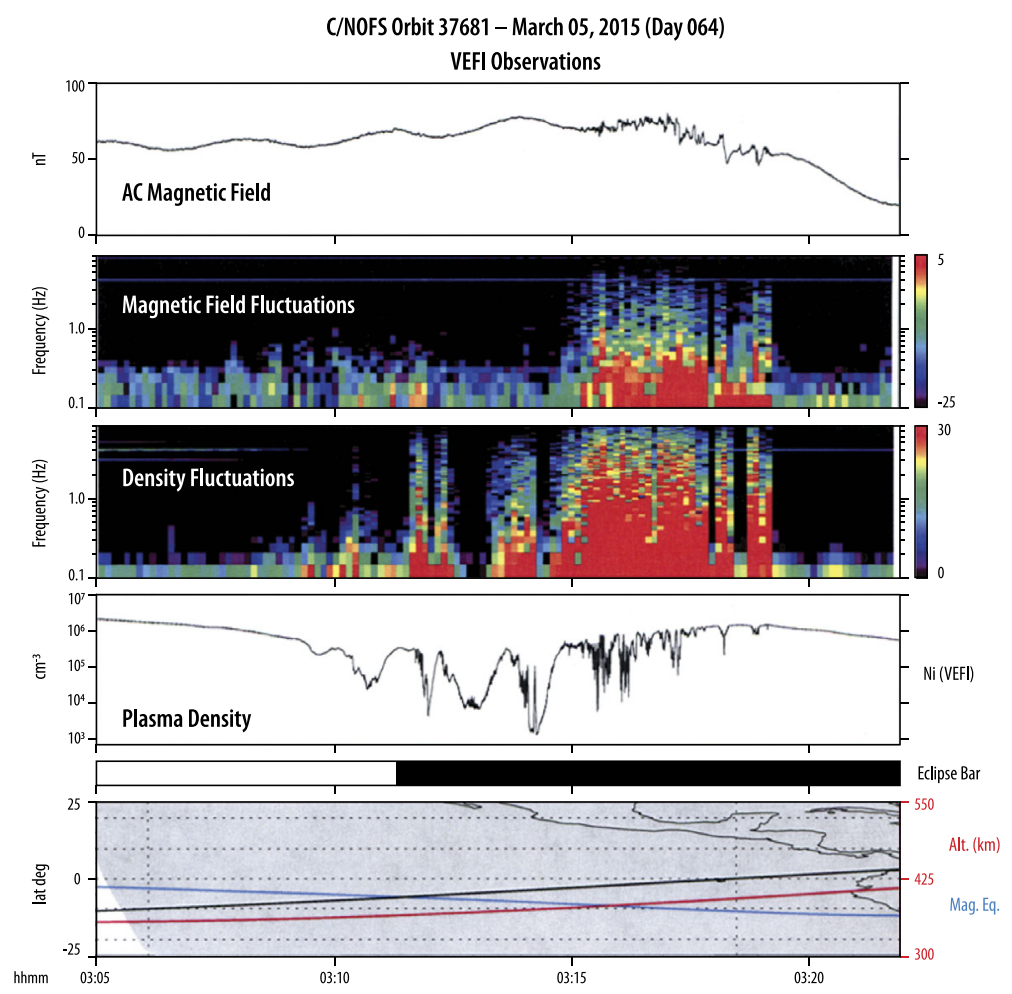

Fig. 70 Example of the AC-coupled magnetic field data showing magnetic field fluctuations associated with density irregularities

The LD digital data include seven amplitude levels in two directions (14 data points comprise one sample) at a cadence of $1.33 \mathrm{~s} / \mathrm{sec}$. Users may thus choose to identify lightning strokes with different amplitudes and compare them to ionospheric electric fields as well as ground-based storm activity. A lightning activity index was computed on the ground for each sample, based on the counts in each energy bin. The geographic location of the composite lightning activity, based on the lightning detector levels, has been reported by Jacobson et al. (2011). An example of the geographic distribution of the lightning detector observations is shown in Fig. 73 which are clearly associated with the specific regions within the continents and agree well with observations from other optical lightning detectors flown in space, such as NASA's TRMM satellite (e.g., Christian et al. 2003).

An example of the time series photometer output is shown in Fig. 74, digitized at $16 \mathrm{ks} / \mathrm{sec}$ as captured in the burst memory. A VLF spectrogram computed on the ground from simultaneous wave electric field data is shown below (Holzworth et al. 2011). Note the strong correlation of the lightning pulses with the VLF sferics and whistlers.

\subsection{Burst Memory Data}

As discussed above, the VEFI burst memory operated in a number of different modes with different trigger inputs. In addition, the number of data channels and their sample rates were highly configurable. Details of which burst modes were operating throughout the mission are available elsewhere. 


\section{C/NOFS lon Density, Orbit 6266, Jun 122009}

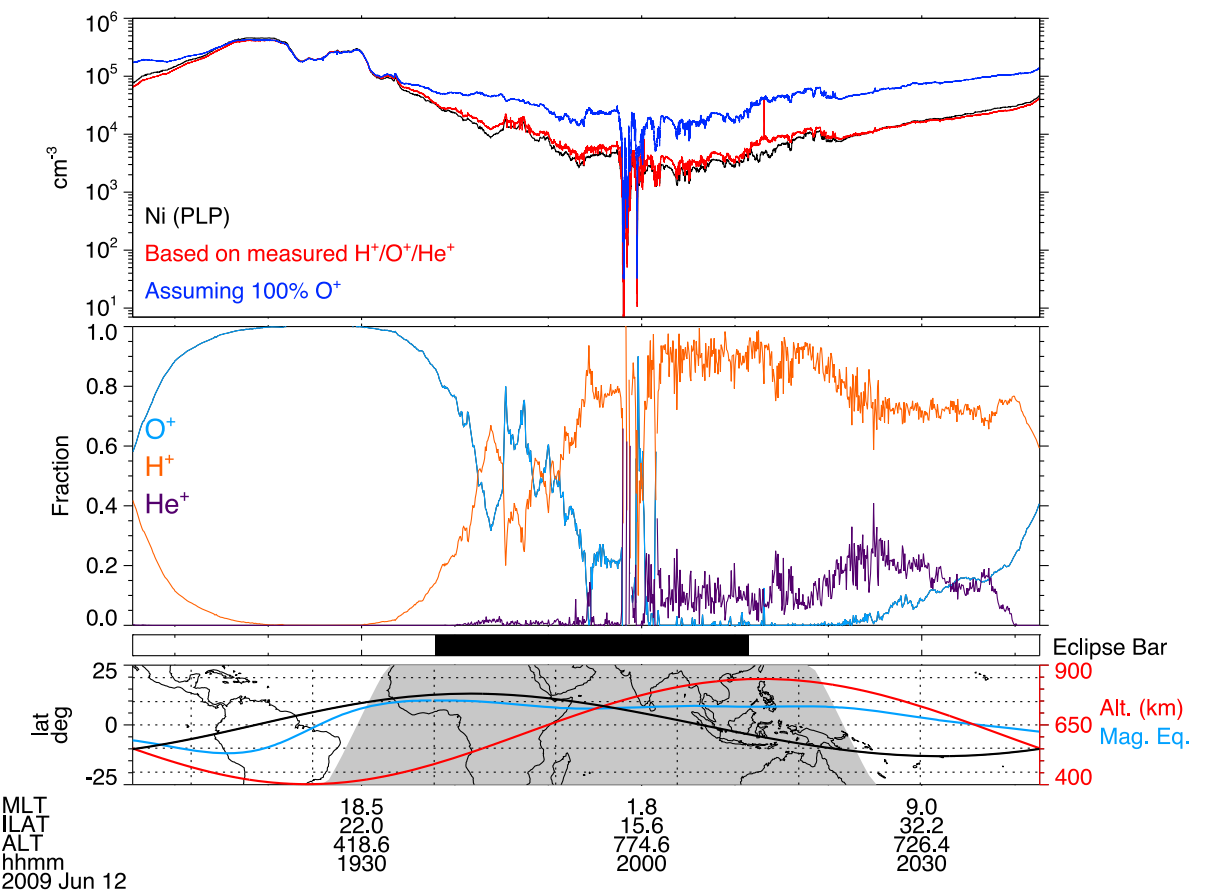

Fig. 71 Plasma density data (top panel) from the Planar Langmuir Probe (PLP) and the VEFI ion density probe for a full orbit centered on midnight. The blue trace shows the density assuming the ambient ions are $100 \% \mathrm{O}^{+}$, and the red curve shows the density using the measured $\mathrm{H}^{+}, \mathrm{He}^{+}$, and $\mathrm{O}^{+}$ion fractions, shown in the middle panel. The lowest panel shows the location of the satellite

In all, over 10,000 bursts were captured and telemetered to the ground. Here we present just a few examples to illustrate some of the key features of the VEFI measurements that are afforded by the instrument's capability to capture high time resolution burst data. The main objective of the burst memory was to capture simultaneous high-time resolution electric field and plasma density oscillations associated with plasma density depletions within the low latitude nighttime ionosphere.

Examples of the VEFI Langmuir probe density data for 6 nighttime orbits are shown in Fig. 75 in which the VEFI burst indicator or flag is shown below the data. The burst indicators show which depletions during the orbit were selected for high-time resolution wave capture. The selection was based on an on-board algorithm within the VEFI instrument which assigned a running "score" of the density data as they were encountered by the spacecraft during the nighttime portion of each orbit. For this particular burst mode, the highest scores were based on the depth and slope of the density depletions. The various features of the scoring algorithm were selectable based on uplinked level criteria and could also be triggered on electric field wave power in a chosen frequency band from the VEFI filter bank. Other modes included a combination of electric field wave power from the filter bank together with the density depletion criteria, as well as other data input and configurations.

Examples of the density and electric field data that were gathered by the burst detector triggering on density depletions are shown in Fig. 76(a, b). For Fig. 76(a), the lowest panel shows the plasma density data sampled at $32 \mathrm{ks} / \mathrm{s}$, revealing both the large-scale structured density as well as density variations superimposed on these data. A spectrogram of these 


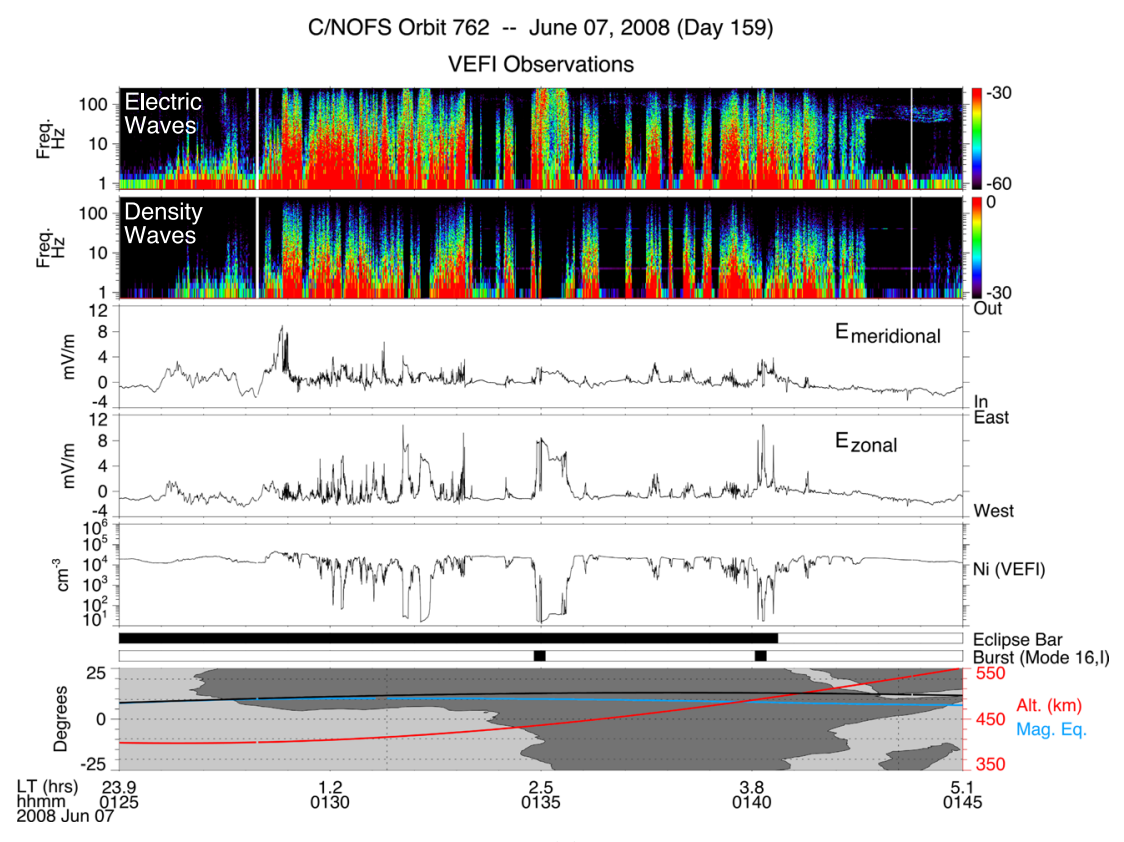

(a)

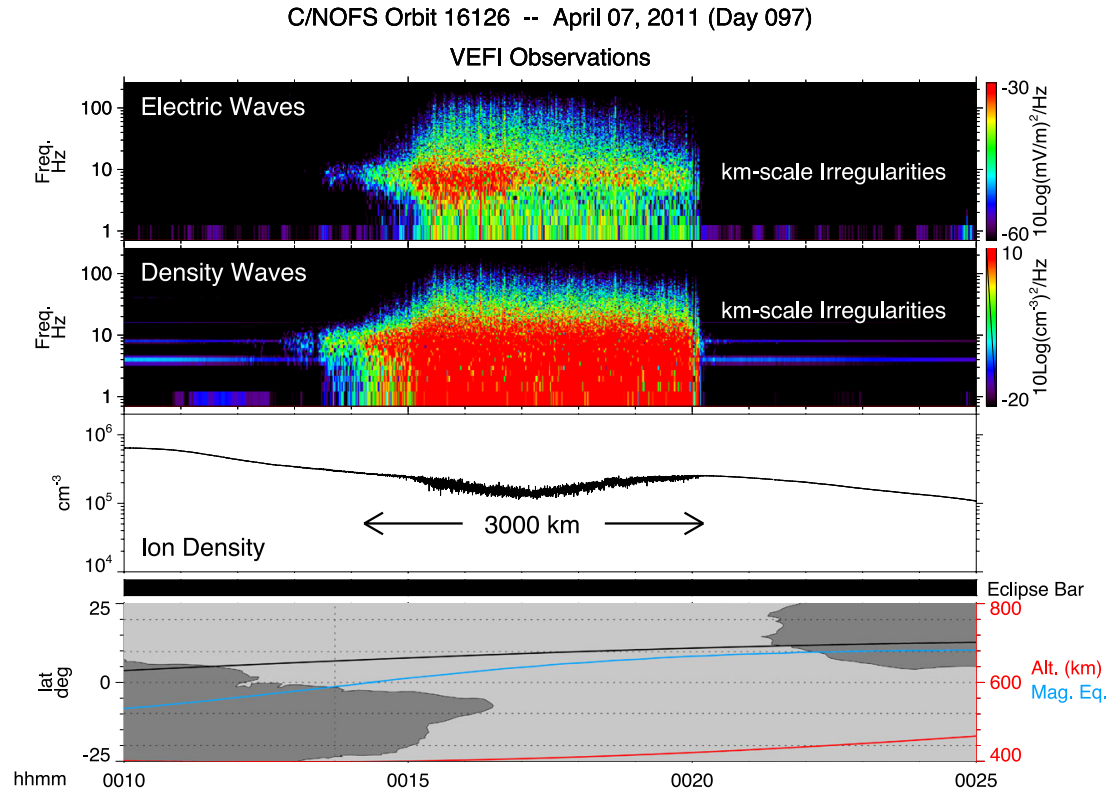

(b)

Fig. 72 (a) Plasma density from the VEFI Langmuir probe (lowest data panel) above which is plotted the DC electric field, showing the strong correspondence of the zonal electric fields and the plasma density depletions. The upper two panels shown the spectrograms of the electric and density data sampled at $512 \mathrm{~s} / \mathrm{sec}$. The lowest panels include the eclipse bar, burst bar, and map showing the C/NOFS location. (b) Plasma density from the VEFI Langmuir probe (lowest data panel) showing a wide swath $(\sim 3000 \mathrm{~km}$ horizontal distance) of $\mathrm{km}$ scale waves appearing near $10 \mathrm{~Hz}$ in the density and electric field spectrograms (upper two panels). The lowest panels include the eclipse bar and map showing the C/NOFS location 


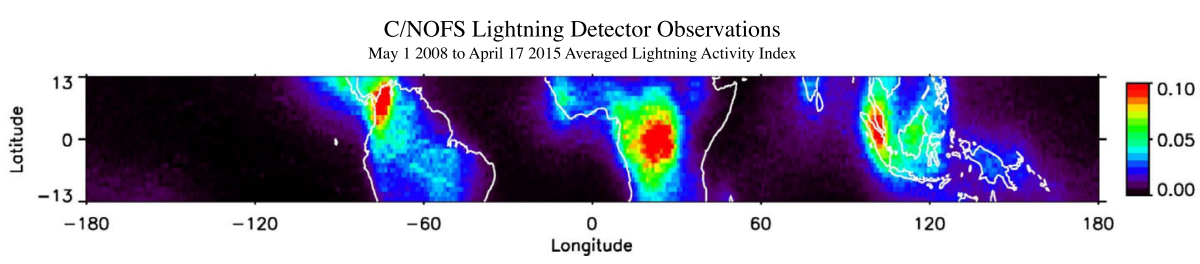

Fig. 73 Average lightning detector counts detected during the C/NOFS mission showing the preponderance of lightning concentrated over specific areas within the continents

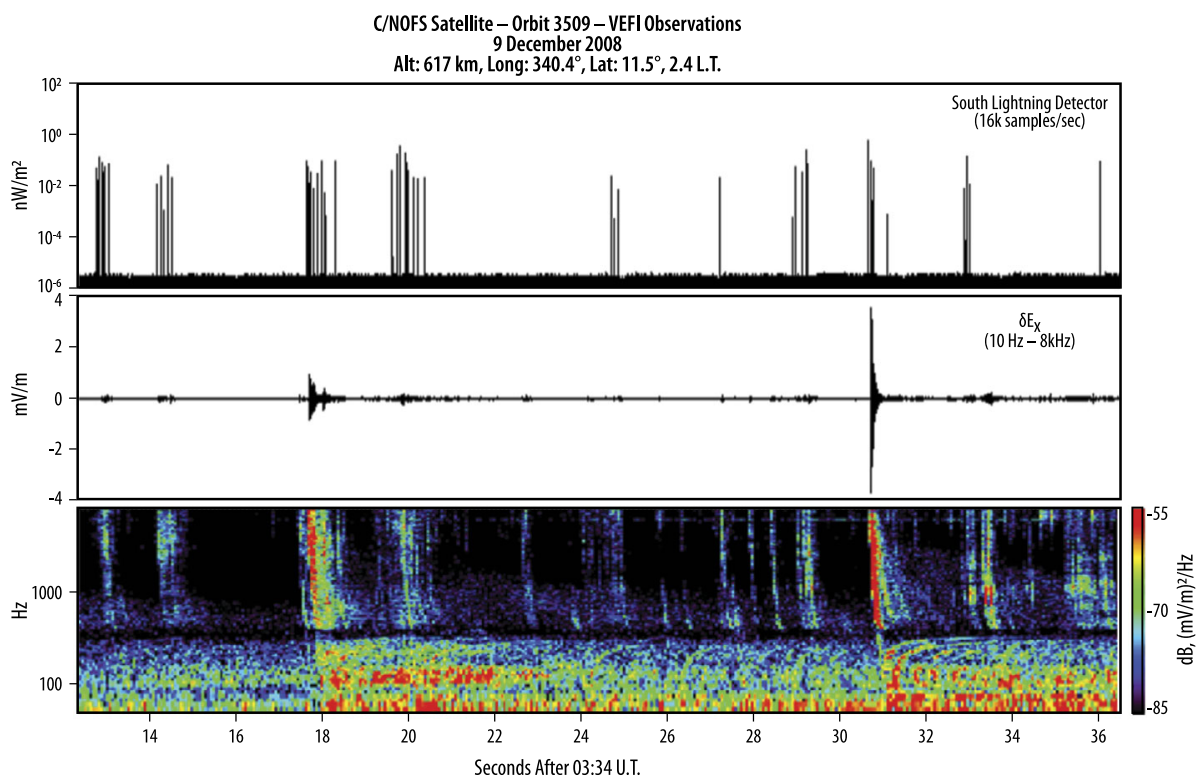

Fig. 74 Optical lightning power (upper panel) and VLF electric field time series (middle panel) captured during a C/NOFS burst mode collection interval. The lowest panel shows a VLF spectrogram computed on the ground of the electric field times series data

data is shown in the panel just above the time series density data. A spectrogram of one of the electric field components is shown above this. Importantly, note that the broad spectrum of irregularities extends to frequencies greater than $1 \mathrm{kHz}$. This example provides clear evidence that the irregularities extended to meter scales (converting the frequencies to scale lengths along the satellite trajectory using the spacecraft velocity of $8 \mathrm{~km} / \mathrm{s}$ ). Besides their scientific importance, identifying scatterers at meter scales is important for scintillation and space weather research and was one of the main reasons for including the burst memory as part of VEFI on C/NOFS.

Another example of wave data captured in a burst is shown in Fig. 76(b). Again, the plasma density data in the lowest panel shows a highly structured ionospheric plasma with strong, clear oscillations near $100 \mathrm{~ms}$ corresponding to $\sim 1 \mathrm{~km}$ scales. The electric field wave data in the spectrogram, plotted above on a linear frequency scale, shows strong VLF waves with a lower frequency cutoff at the lower hybrid frequency that tracks the plasma density and also includes information on the ambient ion composition. The spectrogram also shows the presence of terrestrial VLF transmitters, including some modulated or "keyed" emissions that appear in a periodic fashion. 
C/NOFS VEFI Density Measurements with Burst Mode Triggering Events June 2008
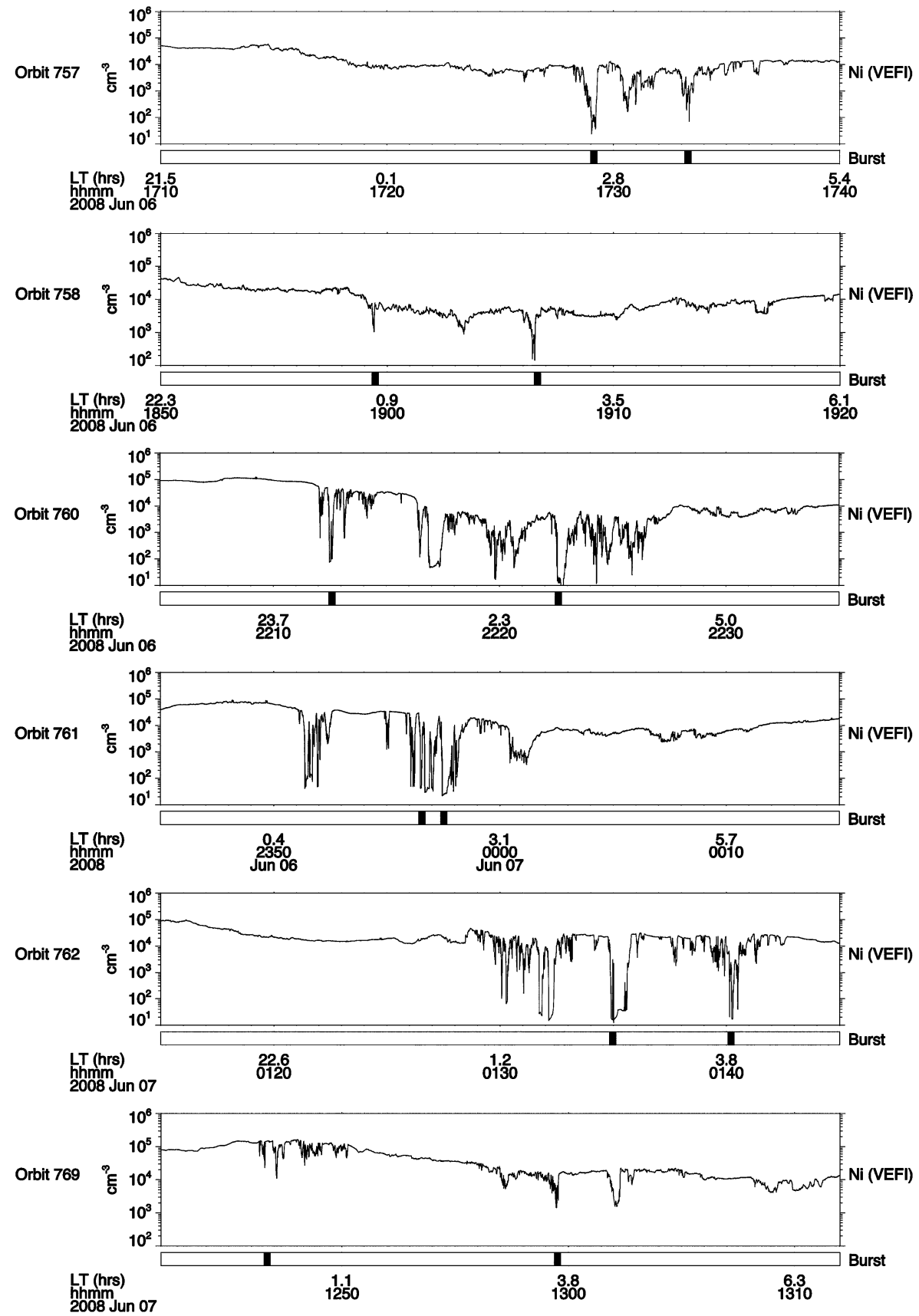

Fig. 75 VEFI plasma density measurements from 6 nighttime passes showing plasma depletions and the intervals where the VEFI burst mode triggered on the depletions (see black indicators or flags in the burst bar beneath each orbit) and subsequently captured high time resolution electric field and density data 


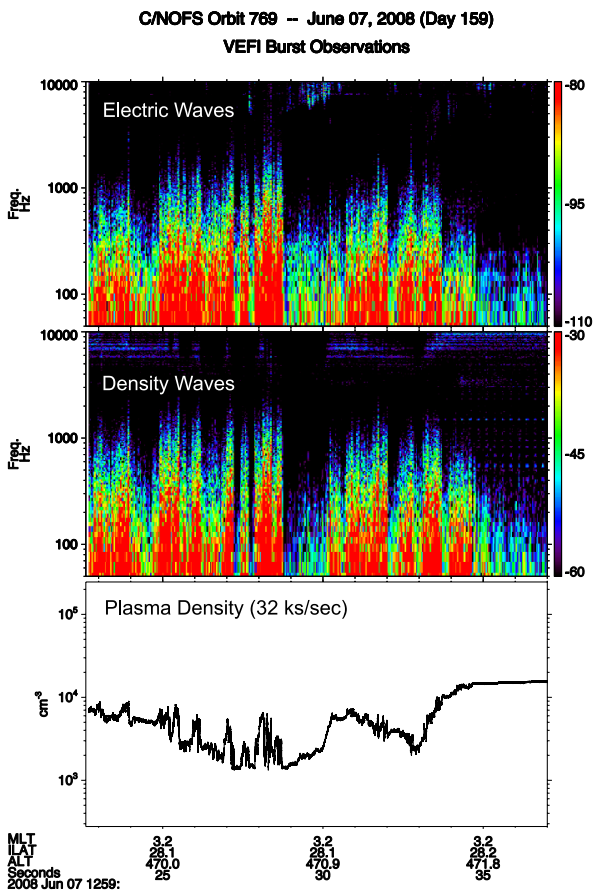

(a)
CNOFS Orbit 757 -- June 06, 2008 (Day 158) VEFI Burst Observations

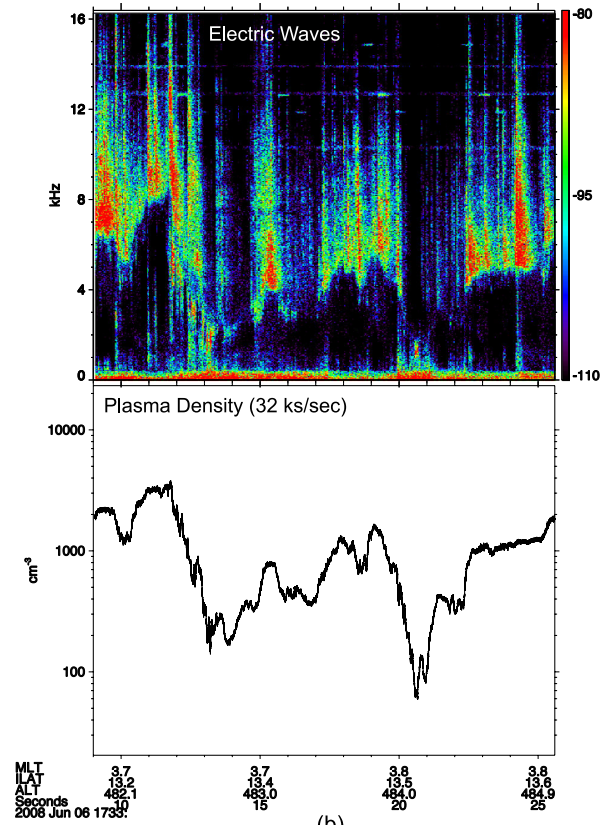

(b)

Fig. 76 Examples of burst data triggered by density depletions, corresponding to two burst intervals in Fig. 75. The data in (a) include a time series of the density data (lowest panel) sampled at $32 \mathrm{ks} / \mathrm{sec}$ and the corresponding spectrogram of these data (middle panel). The upper panel shows a spectrogram of the simultaneous electric field waveform. The lower panel in (b) also shows high time resolution density data which reveal quasi-coherent oscillations with periods near $100 \mathrm{msec}$. A spectrogram of the electric field data (upper panel) reveals sharp cut-offs at the lower hybrid frequency as well as evidence of modulated VLF transmitters detected by the VEFI receiver

The VEFI burst memory also provided a means to capture "interferometer" or spaced receiver data from which the wavelength and phase velocity of irregularities can be obtained (see LaBelle and Kintner 1989, and Pfaff and Marionni 1998, for a discussion of this technique). For C/NOFS, dedicated interferometer "pairs" were formed by AC-coupled potential differences between the outer spheres in the orbit plane and the exposed cylinders along the same axes. As shown in Fig. 77, these pairs permit spaced receiver data in the plane roughly perpendicular to the magnetic field along the orbit. Figure 77 shows spectrograms from each of the two pairs as well as phase and coherency measurements associated with low latitude irregularities. Note how the phase measurements change sign based on the different orientations of the two sets of paired, orthogonal measurements.

As a final example of burst mode data, Fig. 78 shows three components of wave electric field data associated with thunderstorm activity. Here, we have rotated the wave data captured in the burst memory into magnetic coordinates showing all three magnetic components. The lowest panel shows the existence of wave electric fields parallel to the magnetic field direction. As can be seen, such "parallel electric fields" are enhanced near the lower hybrid frequency near $7 \mathrm{kHz}$. Such lower hybrid enhancements associated with lightning sferics have been reported by Kelley et al. (1985) and Berthelier et al. (2008). The data shown here underscore how a tri-axial electric field experiment such as the VEFI experi- 


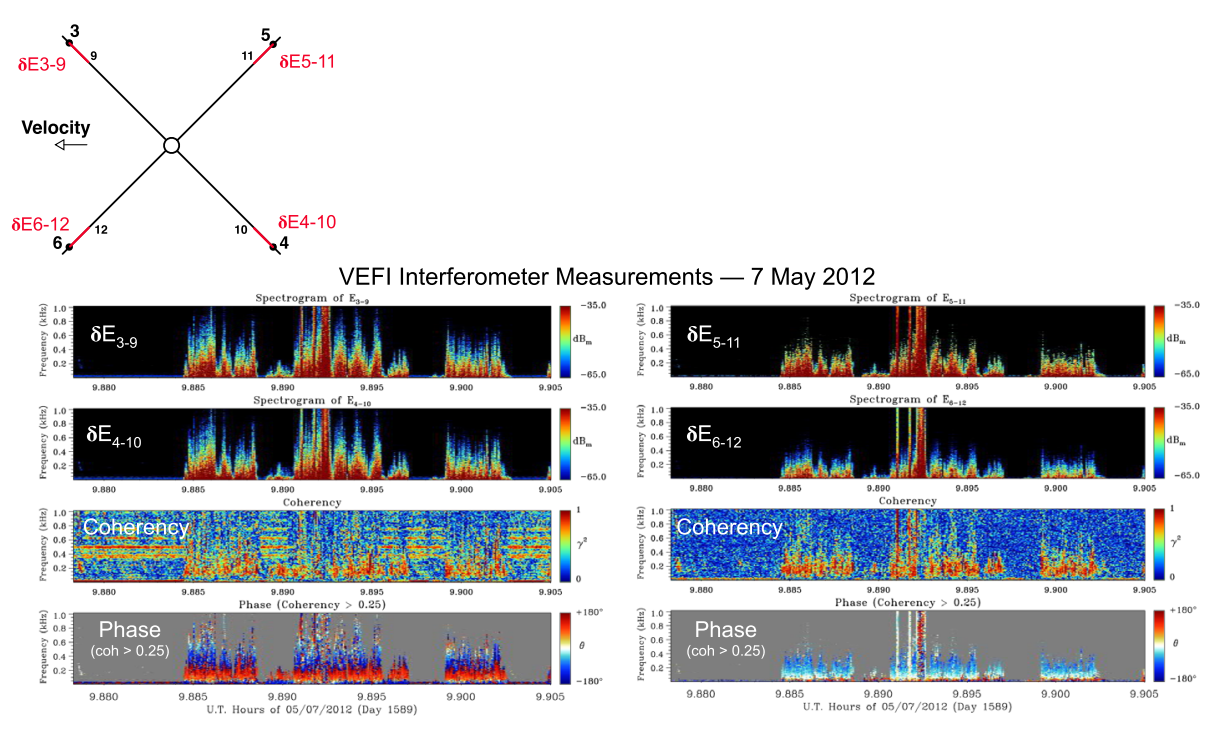

Fig. 77 Burst data of VEFI interferometer electric field measurements during the presence of irregularities. The two pairs of spectrograms (upper panels) show data from the separated probe pairs "3-9" and "4-10" (left) and from the separated probe pairs "5-11" and "6-12" (right). The panels below the spectrograms show the coherency and phase of the two signals. Note the change in phase between the two pairs, in accordance with their different orientations, as shown in the sensor diagram in the upper left

ment on $\mathrm{C} / \mathrm{NOFS}$ enables full vector electric field measurements to be gathered, including those along the magnetic field direction.

\section{Data Processing Notes}

The basic data processing steps for the VEFI data are shown in Fig. 79.

The processing procedures followed standard practices of extracting "Level 0" data from the telemetry data from which it became "Level 1". After the data were decoded using preflight calibration data, these Level 2 "raw" data were time tagged and made available in scientific units (e.g., $\mathrm{mV} / \mathrm{m}, \mathrm{nT}, \mathrm{nA}$, etc.). Analysis procedures then proceeded to produce Level 3 data such as DC electric fields with $\mathbf{V} \times \mathbf{B}$ removed and rotated to magnetic coordinates, magnetometer difference data, etc. The Level 3 data are then available for detailed analysis and study.

After launch and the first month of commissioning, in which VEFI team members were resident at the C/NOFS Data Center at AFRL, VEFI telemetry data were routinely sent to the VEFI Data Processing Center at the NASA/Goddard Space Flight Center on a weekly basis via CD's delivered by the US Post Office.

A standard product of the VEFI analysis was the creation of summary plots of the data from the various sensors. This "quick look" feature enabled the PI and the VEFI team to readily assess the data from each orbit including the status and health of the different sensors. An example is shown in Fig. 80. The data panels from top to bottom are:

- HF spectrogram (on board FFTs),

- VLF spectrogram (on board FFTs),

- V34ELF spectrogram (computed on the ground), 


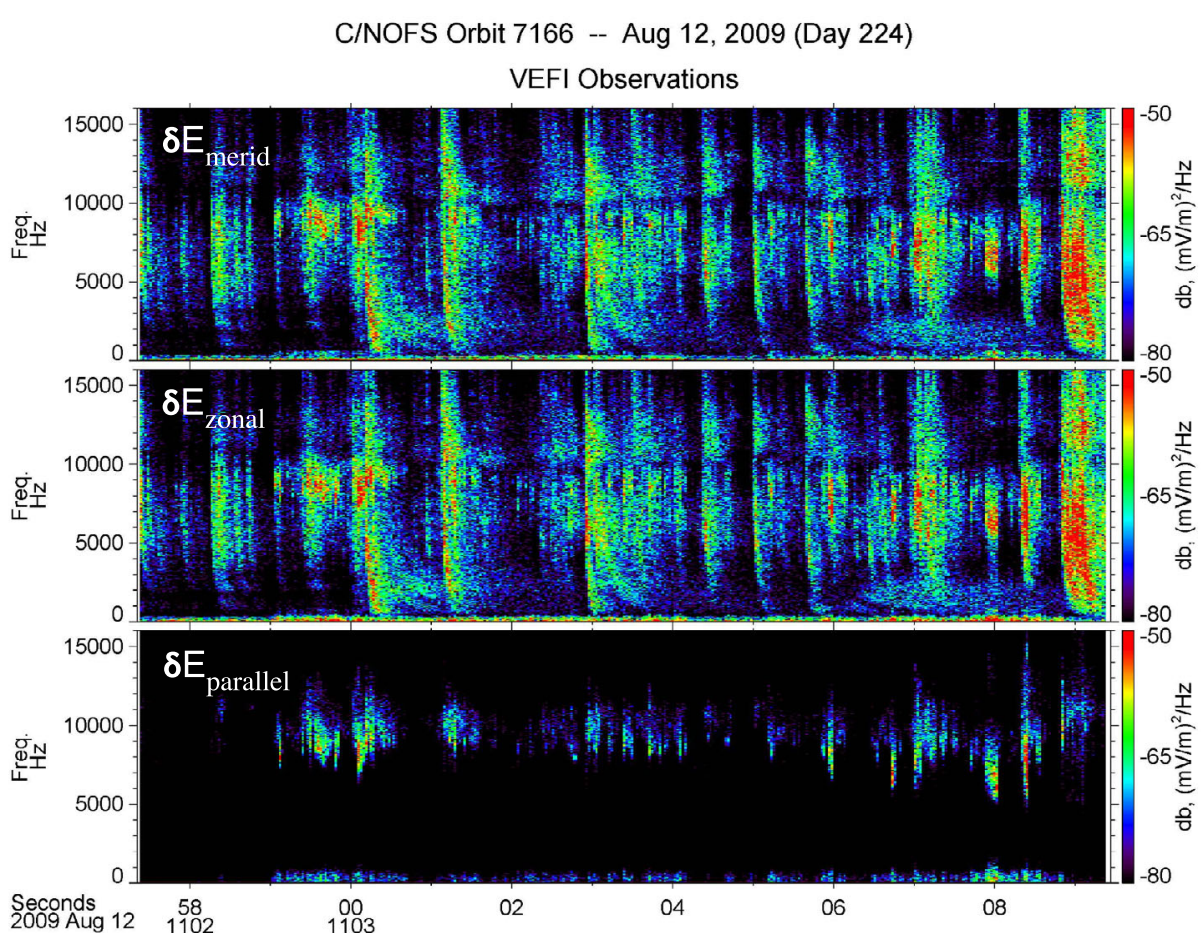

Fig. 78 Burst data of VEFI wave electric fields during a lightning event in which the three measured components have been rotated to geomagnetic coordinates. Note the "parallel" electric fields along the magnetic field direction near the lower hybrid frequency in the lowest panel

- E12, E34, and E56 raw electric field data,

- B1, B2, B3 raw magnetic field data,

- 0-8 Hz spectrogram of the B3 AC magnetic field channel,

- $0-8 \mathrm{~Hz}$ spectrogram of the E34 DC electric field channel,

- 0-8 Hz spectrogram of the Langmuir probe channel,

- Langmuir probe collected current,

- The average of the potentials measured (with respect to the spacecraft) of the six spheres and the average of the potentials measured (with respect to the spacecraft) of the six cylindrical sensors,

- Lightning detector counts for the lowest level from the north and south detectors,

- Eclipse "bar" to show when the spacecraft was occulted by the earth's shadow,

- Burst "bar" to indicate when bursts were acquired with notes on the burst mode,

- Map of low latitudes showing the spacecraft footprint in black with the magnetic equator shown in blue for reference. The spacecraft altitude is shown in red. The grey shading shows the portion of the orbit in which the earth was in shadow on the ground.

\section{Summary}

This article has described the various sensors that constitute the VEFI experiment suite and has discussed their operation during the C/NOFS mission. As reported here, the Vector 


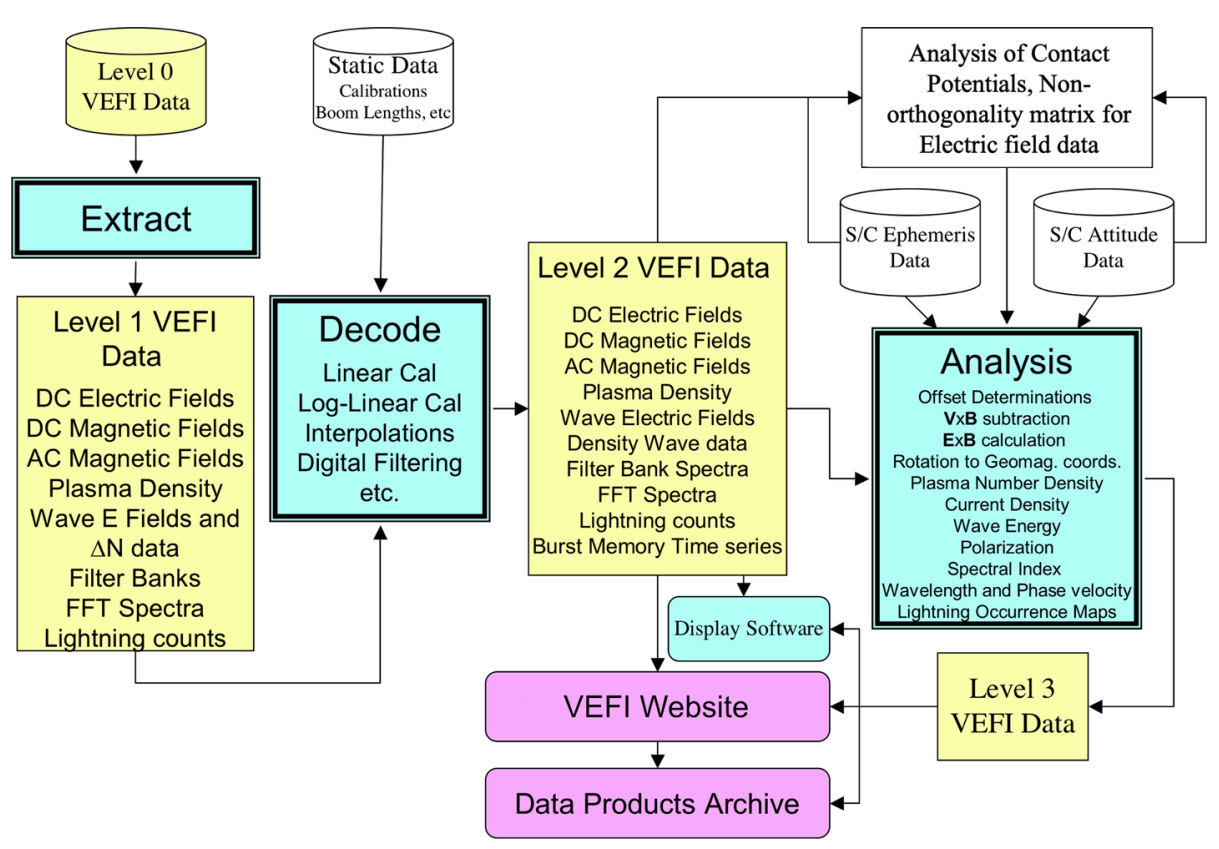

Fig. 79 VEFI data processing flow diagram showing the progression of the raw data through the Level 1, Level 2, and Level 3 stages

Electric Field Investigation (VEFI) on the C/NOFS satellite comprises a suite of sensors controlled by one central electronics box. The primary measurement consists of a vector electric field detector which extends spherical sensors with embedded pre-amps at the ends of six, 9.5-m booms to form a system of three orthogonal electric field detectors with baselines of $20 \mathrm{~m}$ tip-to-tip each. The electric field detector is configured to provide both DC and $\mathrm{AC}$ (or wave) electric fields, which includes a wide range of plasma waves ranging from ULF to HF frequencies which were gathered with the same sensors. VEFI includes a flux-gate magnetometer that provided data at 1 vector/sec and an AC-coupled magnetic field measurement provided at 16 vectors/sec.

VEFI includes a fast, fixed-bias Langmuir probe that serves as the input signal to trigger the VEFI burst memory collection of high time resolution electric field and plasma wave data when plasma density depletions are encountered in the low latitude nighttime ionosphere. A bi-directional optical lightning detector designed by the Univ. of Washington provides continuous average lightning counts at different optical irradiance levels as well as high time resolution optical lightning emissions captured in the burst memory.

VEFI electronics included a highly configurable, programmable burst memory with inputs from multiple sensor types which allows for different trigger inputs, channels, and sampling rates. VEFI also accommodated different telemetry rates throughout the mission.

Examples of data included in this article illustrate the performance of the different sensors in space. Included herein is a discussion of the different techniques used to determine the electric field and magnetic field offsets on a non-spinning satellite.

VEFI included a number of technical advances and innovative features which are described in this article and summarized below: 


\section{C/NOFS Orbit 11601 -- June 06, 2010 (Day 157)}

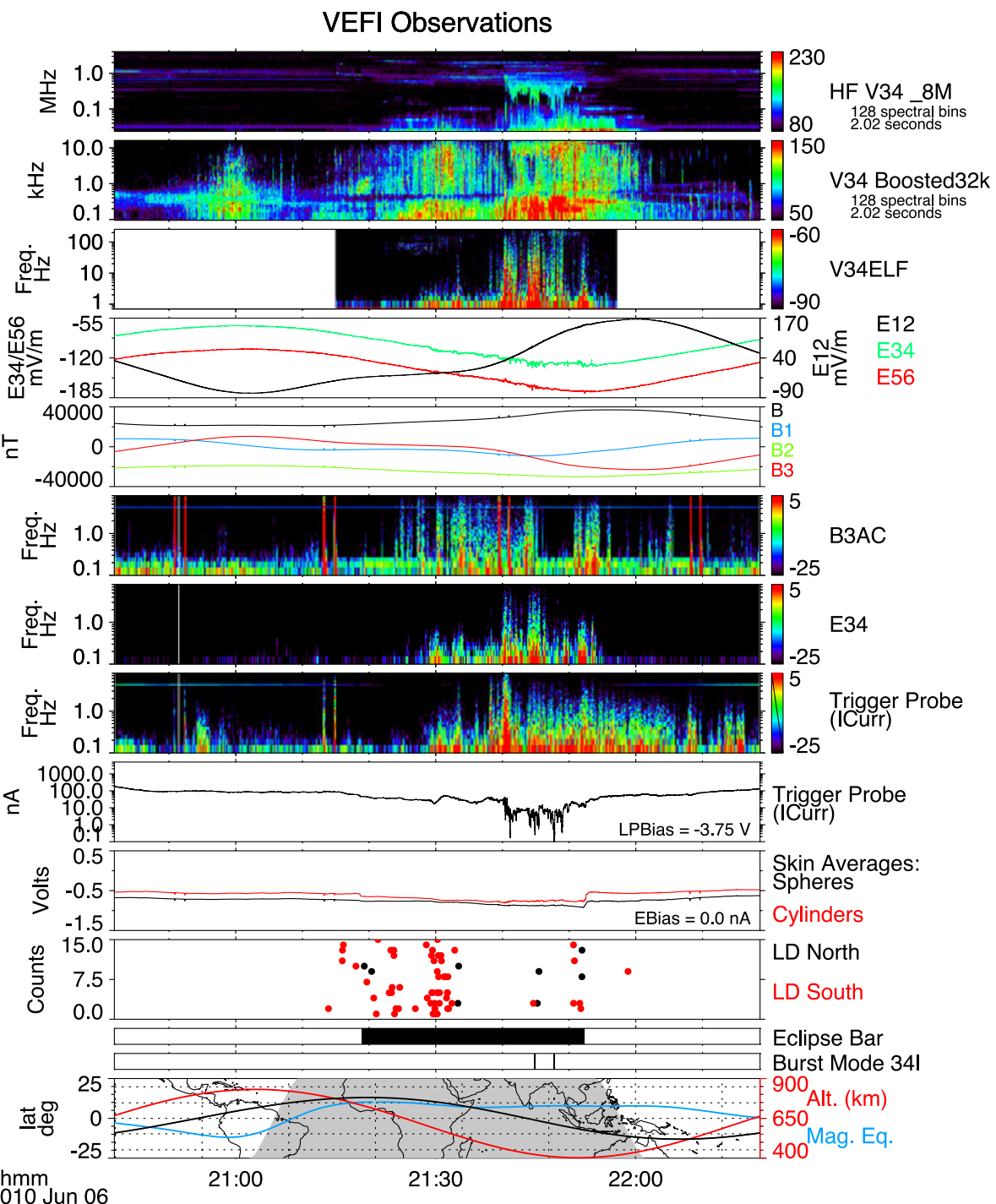

Fig. 80 Example of a VEFI Summary plot for one orbit showing examples of the different types of VEFI data displayed versus time

- First dual electric field experiment flown on a satellite with two independent sets of 3-axis, orthogonal double probes

- Motor-driven, pre-formed cylinder booms with signal wires in the center that fed preamps within spherical sensors at the ends of the booms

- Extended shadow equalizers (2.5 times the sphere diameter) to mitigate photoelectron shadow mismatch for sun angles along the boom directions in the orbit plane, particularly important at sunrise/sunset for a low inclination satellite 
- Main electronics consisting of a "box within a box", with tightly closed trays for EMI control

- DC-coupled electric field channels with "boosted" or pre-emphasized amplitude response at ELF frequencies

- Configurable wave data including broadband and on-board FFT response with selectable frequency bins for transmission

- Magnetometer booms fabricated entirely of Zelux material with $40 \%$ glass

- Miniature multi-channel spectrum analyzers using hybrid technology

- Dual-channel optical lightning detector with on-board comparators and counters for 7 amplitude irradiance levels as well as high-time-resolution data capture

- Spherical Langmuir probe with Titanium Nitride sensor element and guard

- Fixed-bias Langmuir probe designed for very low currents such as those corresponding to ionospheric plasma (spread-F) depletions with densities less than $10^{2} / \mathrm{cm}^{3}$ including temperature compensation electronics

- Data supported different data rates, including $200 \mathrm{kbps}$ (fast), $20 \mathrm{kbps}$ (nominal), and 2 kbps (low for real-time TDRSS communication)

- Oversampled, averaged DC electric and magnetic field survey data, with 24-bit counters telemetered for the magnetometer data to enhance its LSB sensitivity

- Highly configurable burst memory with selectable channels, sample rates and number, duration, and precursor length of bursts, chosen based on best "score" of triggering algorithm each orbit

- Tailored burst memory trigger algorithms that included density depletions, wave power amplitude from any of 12 filter bank channels, optical lightning irradiance, a combination of these, or a pre-determined time (e.g., when the satellite traversed a ground-based radar)

- Entire VEFI Instrument, including all sensors and electronics, was designed, built, tested, and delivered on budget in less than 2 years

The data show that all six VEFI booms deployed nominally and remained in their orthogonal configuration throughout the mission. Furthermore, the VEFI measurements shown here demonstrate that the DC and wave electric field data are of high quality, as are the data from the VEFI magnetometer, Langmuir probe, and lightning detector.

\section{Appendix: Electric Field Sensor Temperatures}

We present the temperatures within the electric field spheres as measured during the $\mathrm{C} / \mathrm{NOFS}$ mission. The electronics board within each electric field sphere included a temperature sensor from which the temperature of that board was monitored. The signal was sent down via a wire within the ribbon cable routed through the boom elements and were subsequently digitized within the VEFI electronics box. The temperatures provide a useful diagnostic regarding the health of the pre-amp environment of each sphere as well as information that pertains to the outer sphere coating.

Figure 81 shows the temperatures of the sphere pre-amp electronics boards for the entire mission. In general, the temperatures were maintained between -15 and $+30 \operatorname{deg} \mathrm{C}$ with daily averages temperatures between roughly 0 and $20 \mathrm{deg}$ C. It is unclear why the temperatures for sphere electronics boards 1 and 2 were slightly higher than those of other spheres. Similarly, the somewhat lower temperature of the sphere \#6 electronics board is not understood. The temperatures for all of the electronics boards increased after a five-month mission hiatus in 2013, in which the instruments were powered down and the spacecraft was put in safehold. The temperatures increased by about 10 degrees during the last year of 

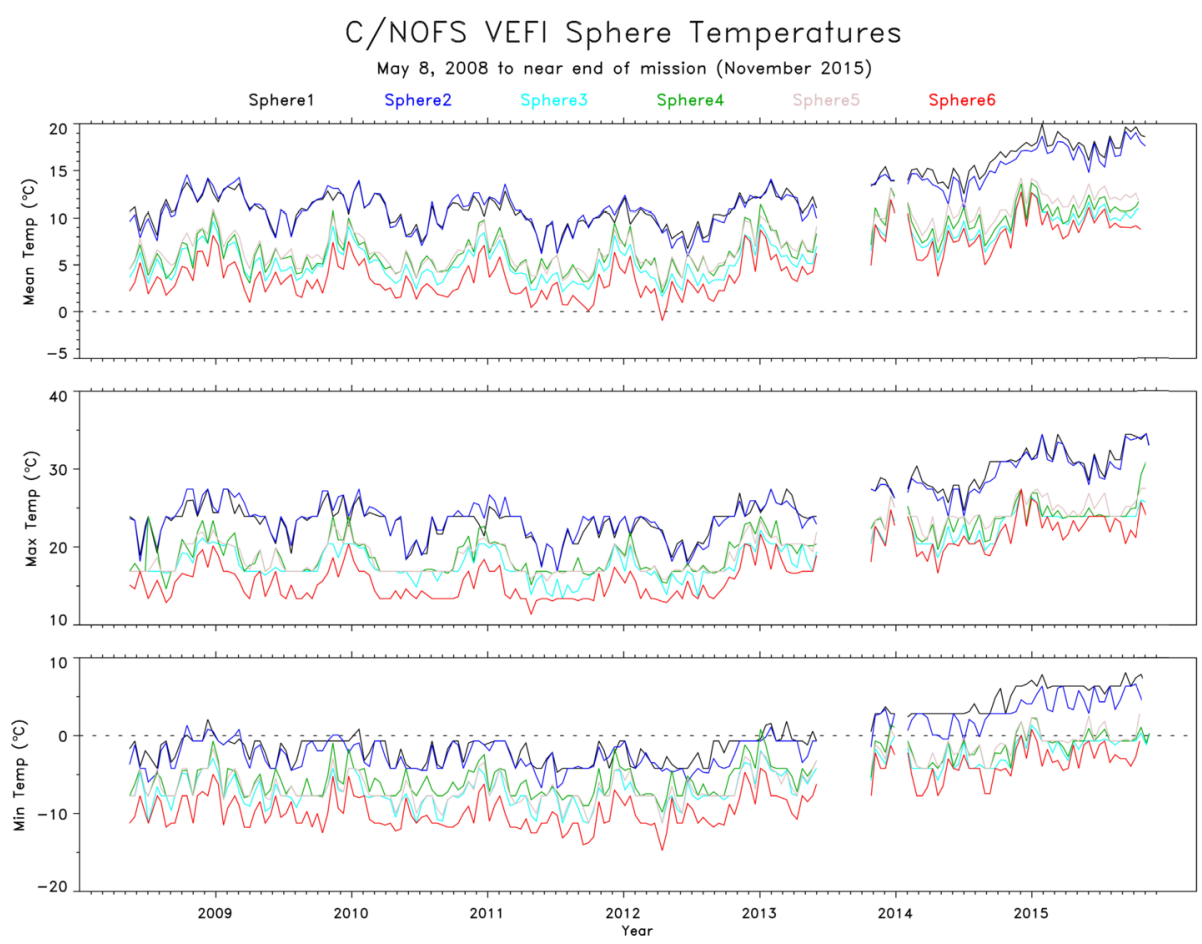

Fig. 81 Measured sphere temperatures for the entire C/NOFS orbit showing the mean, maximum, and minimum temperatures averaged for each orbit. Note the different scales of the panels

the mission, either due to increased neutral density environment as the spacecraft altitude slowly decreased, or changes in the outer carbon coating. Indeed, the DAG213 coating may have eroded during this time due to the atomic oxygen environment, which might have also accounted for the increased temperatures.

Daily temperatures are shown in Fig. 82 for one day in 2008 and one day in 2015 (15 orbits per day). Note the diurnal temperature variations each sphere experienced during each orbit between day and night conditions.

The continuous, daily, variations of the measured temperatures of the electronics boards within the six spheres are in agreement with thermal analysis provided prior to launch. The general agreement between all of the sphere temperatures supports the overall conclusion that the electric field sphere environments were nominal throughout the 7.5 year mission.

Acknowledgements We thank the reviewers for their useful comments. We acknowledge helpful comments from Dr. John Bonnell and Dr. Harri Laakso.

The C/NOFS mission, conceived and developed by the Air Force Research Laboratory (AFRL), was sponsored and executed by the USAF Space Test Program. We acknowledge, with thanks, the project leadership at AFRL, in particular Dr. Odile de la Beaujardière, Dr. Donald Hunton, and Ms. Louise Gentile. The VEFI instrument at Goddard relied on a highly dedicated team and support staff which encompassed all aspects of the instrument design, fabrication, and test, the detailed processing and analysis of the data, and the management and stewardship of personnel and resources. We salute in particular the dedicated support and expert contributions of Ms. Debbie Chipouras of Goddard's Electric Field group. 


\section{C/NOFS VEFI Sphere Temperatures}

May 08, 2008

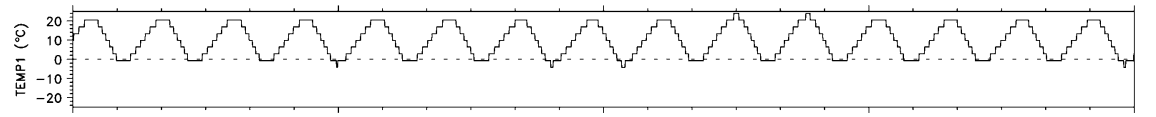

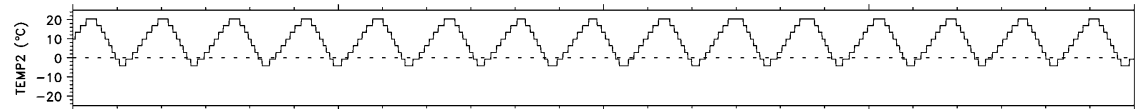

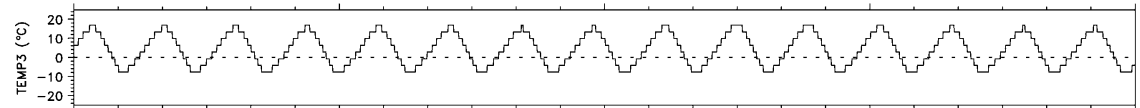

E

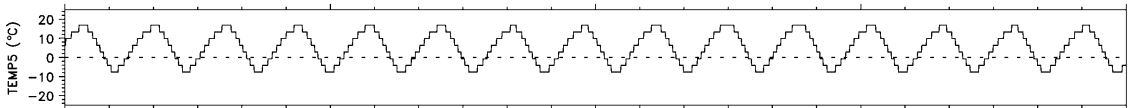

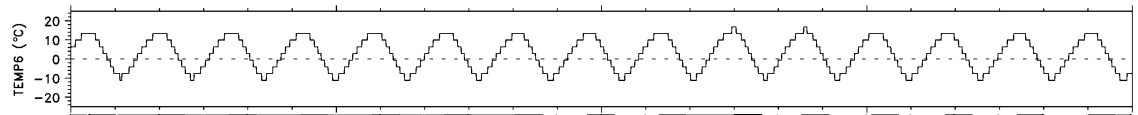

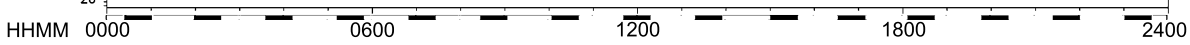

August 31, 2015

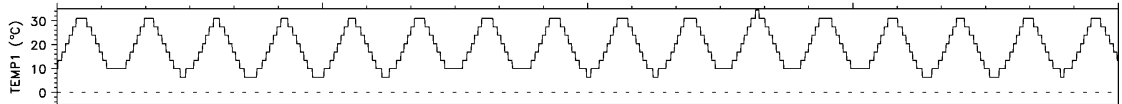

: MNANNNNNNA

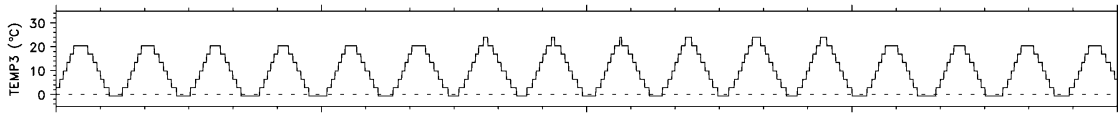

PHANANANANAN

EMANANANANA

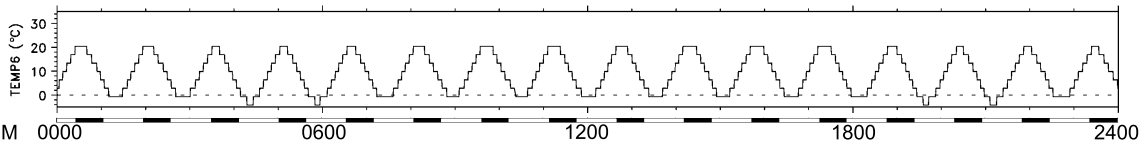

Fig. 82 Sphere temperatures for two days (15 orbits each) of the C/NOFS mission. The data on the top correspond to near the beginning of the mission (May 8, 2008) and the data on the bottom correspond to a day towards the end of the mission (August 31, 2015). The diurnal variations from when the satellite was solar illuminated and when it was in darkness, as shown by the eclipse intervals in the bottom bar, are clearly evident 


\section{Declarations}

Conflict of Interest Authors declare no competing interests.

Open Access This article is licensed under a Creative Commons Attribution 4.0 International License, which permits use, sharing, adaptation, distribution and reproduction in any medium or format, as long as you give appropriate credit to the original author(s) and the source, provide a link to the Creative Commons licence, and indicate if changes were made. The images or other third party material in this article are included in the article's Creative Commons licence, unless indicated otherwise in a credit line to the material. If material is not included in the article's Creative Commons licence and your intended use is not permitted by statutory regulation or exceeds the permitted use, you will need to obtain permission directly from the copyright holder. To view a copy of this licence, visit http://creativecommons.org/licenses/by/4.0/.

\section{References}

M.H. Acuña, Fluxgate magnetometers for outer planet exploration. IEEE Trans. Magn. 10, 519-523 (1974)

J.J. Berthelier, M. Godefroy, F. Leblanc, M. Malingre, M. Menvielle, D. Lagoutte et al., ICE, the electric field experiment on DEMETER. Planet. Space Sci. 54(5), 456-471 (2006)

J.J. Berthelier et al., Lightning-induced plasma turbulence and ion heating in equatorial ionospheric depletions. Nat. Geosci. 1, 101-105 (2008)

L.H. Brace, Langmuir probe measurements in the ionosphere, in Measurement Techniques in Space Plasmas: Particles, vol. 34. Geophysical Monograph, vol. 102 (1998)

L. Chen, R. Pfaff, R. Heelis, S. Boardsen, Z. Xia, Ion cyclotron resonant absorption lines in ELF hiss power spectral density in the low-latitude ionosphere. Geophys. Res. Lett. (2020). https://doi.org/10.1029/ 2019GL086315

H.J. Christian et al., Global frequency and distribution of lightning as observed from space by the Optical Transient Detector. J. Geophys. Res. 108(D1), 4005 (2003). https://doi.org/10.1029/2002JD002347

O. de La Beaujardière, L. Jeong, B. Basu, S. Basu, T. Beach, P. Bernhardt et al., C/NOFS: a mission to forecast scintillations. J. Atmos. Sol.-Terr. Phys. 66(17 SPEC. ISS.), 1573-1591 (2004)

U. Fahleson, Theory of electric field measurements conducted in the magnetosphere with electric probes. Space Sci. Rev. 7(2-3), 238-262 (1967)

B.G. Fejer, B.D. Tracy, R.F. Pfaff, Equatorial zonal plasma drifts measured by the C/NOFS satellite during the 2008-2011 solar minimum. J. Geophys. Res. Space Phys. 118(6), 3891-3897 (2013)

D.C. Ferguson, D. Cooke, R. Pfaff, D. Rowland, J. Klenzing, H. Freudenreich, Ram/wake and surface layer effects on DC electric field measurements in LEO. IEEE Trans. Plasma Sci. 41, 12 (2013)

H. Freudenreich, R. Pfaff, Processing and analysis of the flux-gate magnetometer data gathered on the C/NOFS satellite (2021). Submitted

D. Gurnett et al., The Cassini radio and plasma wave investigation. Space Sci. Rev. 114, 395-463 (2004)

W.B. Hanson, W.R. Coley, R.A. Heelis, A comparison of in situ measurements of E $\searrow$ and $-\mathrm{V} \searrow \times B \searrow$ from Dynamics Explorer 2. J. Geophys. Res. 98, A12 (1993)

R.A. Heelis, W.B. Hanson, Measurements of thermal ion drift velocity and temperature using planar sensors, in Measurement Techniques in Space Plasmas: Particles, ed. by R.F. Pfaff, J.E. Borovsky, D.T. Young. Geophys. Monogr. Ser., vol. 102 (AGU, Washington, 1998), pp. 61-71

R. Holzworth, M. Kelley, C. Siefring, L. Hale, J. Mitchell, Electrical measurements in the atmosphere and the ionosphere over an active thunderstorm: 2. Direct current electric fields and conductivity. J. Geophys. Res. 90(A10), 9824-9830 (1985)

R.H. Holzworth, K.W. Norville, P.M. Kintner, S.P. Powell, Stratospheric conductivity variations over thunderstorms. J. Geophys. Res. 91(D12), 13257-13263 (1986)

R.H. Holzworth, R.M. Winglee, B.H. Barnum, Y. Li, M.C. Kelley, Lightning whistler waves in the high-latitude magnetosphere. J. Geophys. Res. 104, 17,369-17,378 (1999). https://doi.org/10.1029/ 1999JA900160

R.H. Holzworth, M.P. McCarthy, R.F. Pfaff, A.R. Jacobson, W.L. Willcockson, D.E. Rowland, Lightninggenerated whistler waves observed by probes on the Communication/Navigation Outage Forecast System satellite at low latitudes. J. Geophys. Res. 116, A06306 (2011)

D.L. Hysell, An overview and synthesis of plasma irregularities in equatorial spread F. J. Atmos. Sol.-Terr. Phys. 62, 1037-1056 (2000)

D.L. Hysell, R.B. Hedden, J.L. Chau, F.R. Galindo, P.A. Roddy, R.F. Pfaff, Comparing F region ionospheric irregularity observations from C/NOFS and Jicamarca. Geophys. Res. Lett. 36(18), 1-5 (2009) 
K.S. Jacobsen, J.-E. Wahlund, A. Pedersen, Cassini Langmuir probe measurements in the inner magnetosphere of Saturn. Planet. Space Sci. 57, 48-52 (2009)

A.R. Jacobson, R.H. Holzworth, M.P. McCarthy, R.F. Pfaff, Initial studies with the lightning detector on the C/NOFS satellite, and cross validation with WWLLN. J. Atmos. Ocean. Technol. 28(11), 1423-1435 (2011)

M.C. Kelley, C.L. Siefring, R.F. Pfaff, P.M. Kintner, M. Larsen, R. Green et al., Electrical measurements in the atmosphere and the ionosphere over an active thunderstorm: 1. Campaign overview and initial ionospheric results. J. Geophys. Res. 90(A10), 9824 (1985)

M.C. Kelley, J.G. Ding, R.H. Holzworth, Intense ionospheric electric and magnetic field pulses generated by lightning. Geophys. Res. Lett. 17, 2221-2224 (1990). https://doi.org/10.1029/GL017i012p02221

J. Klenzing, D. Rowland, The fixed-bias Langmuir probe on the Communication/Navigation Outage Forecast System satellite: calibration and validation. Rev. Sci. Instrum. 83, 114501 (2012)

J. Klenzing, F. Simões, S. Ivanov, R.A. Heelis, D. Bilitza, R. Pfaff, D. Rowland, Topside equatorial ionospheric density and composition during and after extreme solar minimum. J. Geophys. Res. Space Phys. 116(12), 1-10 (2011). https://doi.org/10.1029/2011JA017213

E. Kudeki, A. Akgiray, M. Milla, J.L. Chau, D.L. Hysell, Equatorial spread-F initiation: post-sunset vortex, thermospheric winds, gravity waves. J. Atmos. Sol.-Terr. Phys. 69(17-18), 2416-2427 (2007)

H. Laakso, T.L. Aggson, R.F. Pfaff, Plasma gradient effects on double-probe measurements in the magnetosphere. Ann. Geophys. 13, 130 (1995)

J. LaBelle, P.M. Kintner, The measurement of wavelength in space plasmas. Rev. Geophys. 2(7), 495-518 (1989)

Y.Q. Li, R.H. Holzworth, H. Hu, M. McCarthy, R.D. Massey, P.M. Kintner, J.V. Rodrigues, U.S. Inan, W.C. Armstrong, Anomalous optical events detected by rocket-borne sensor in the WIPP campaign. J. Geophys. Res. 96, 1315-1326 (1991). https://doi.org/10.1029/90JA01727

H. Lühr, S. Maus, M. Rother, Noon-time equatorial electrojet: its spatial features as determined by the CHAMP satellite. J. Geophys. Res. 109, A01306 (2004)

N.C. Maynard, Electric Field Measurements in Moderate to High Density Space Plasmas with Passive Double Probes. Geophysical Monograph Series, vol. 103 (1998)

N.C. Maynard, E.A. Bielecki, H.F. Burdick, Instrumentation for vector electric field measurements from DEB. Space Sci. Instrum. 5, 523-534 (1981)

N.C. Maynard, J.P. Heppner, A. Egeland, Intense, variable electric fields at ionospheric altitudes in the high latitude regions as observed by DE-2. Geophys. Res. Lett. 9(9), 981-984 (1982)

N.C. Maynard, T.L. Aggson, F.A. Herrero, M.C. Liebrecht, Average low-latitude meridional electric fields from DE 2 during solar maximum. J. Geophys. Res. 93(A5), 4021-4037 (1988)

N.C. Maynard, T.L. Aggson, F.A. Herrero, M.C. Liebrecht, J.L. Saba, Average equatorial zonal and vertical ion drifts determined from San Marco D electric field measurements. J. Geophys. Res. 100(A9), 17465-17479 (1995)

F.S. Mozer, Electric fields, in Geoscience Instrumentation, ed. by E.A. Wolf, E.P. Mercanti (Wiley and Sons, New York, 1971), pp. 255-267

R. Pfaff et al., Critical analysis of the DC electric field detector on the C/NOFS satellite (2021). Submitted

R. Pfaff, P.A. Marionni, Multiple-baseline spaced receivers, in Measurement Techniques in Space Plasmas: Fields. AGU Geophysical Monograph, vol. 103 (1998)

R.F. Pfaff, In-situ measurement techniques for ionospheric research, in Modern Ionospheric Science, ed. by H. Kohl et al. (Max-Planck-Institut für Aeronomie, Katlenburg-Lindau, 1996)

R. Pfaff, D. Rowland, H. Freudenreich, K. Bromund, G. Le, M. Acuña et al., Observations of DC electric fields in the low-latitude ionosphere and their variations with local time, longitude, and plasma density during extreme solar minimum. J. Geophys. Res. Space Phys. 115(12), 1-12 (2010a)

R. Pfaff, P. Schuck, J. Klenzing, VLF and HF plasma waves associated with spread-F plasma depletions observed on the C/NOFS satellite. Abstract, URSI Meeting USA (2010b)

R. Pfaff, H. Freudenreich, D. Rowland, J. Klenzing, C. Liebrecht, Electric field and plasma density observations of irregularities and plasma instabilities in the low latitude ionosphere gathered by the C/NOFS satellite. Abstract, ISEA Meeting, Peru (2013a)

R. Pfaff, C. Liebrecht, H. Freudenreich, J. Klenzing, Large expanses of kilometer-scale waves predominantly observed below the F-peak encountered by the electric field and plasma density probes on the C/NOFS satellite. Abstract, Fall, AGU (2013b)

R. Pfaff, H. Freudenreich, G. Le, Ionospheric currents observed using the flux-gate magnetometer on the C/NOFS satellite. Abstract, Fall, AGU (2018)

J.M. Retterer, D.T. Decker, W.S. Borer, R.E. Daniell Jr., B.G. Fejer, Assimilative modeling of the equatorial ionosphere for scintillation forecasting: modeling with vertical drifts. J. Geophys. Res. 110, A11307 (2005) 
P.A. Roddy, D.E. Hunton, J.O. Ballenthin, K.M. Groves, Correlation of in situ measurements of plasma irregularities with ground-based scintillation observations. J. Geophys. Res. 115, A06303 (2010). https:// doi.org/10.1029/2010JA015288

F. Simões, R. Pfaff, H. Freudenreich, Satellite observations of Schumann resonances in the Earth's ionosphere. Geophys. Res. Lett. 38, L22010 (2011)

C. Stolle, H. Lühr, M. Rother, G. Balasis, Magnetic signatures of equatorial spread F as observed by the CHAMP satellite. J. Geophys. Res. Space Phys. 111(2), 1-13 (2006)

R.T. Tsunoda, R.C. Livingston, J.P. McClure, W.B. Hanson, Equatorial plasma bubbles: vertically elongated wedges from the bottomside F layer. J. Geophys. Res. 87(A11), 9171 (1982)

C.E. Valladares, W.B. Hanson, J.P. McClure, B.L. Cragin, Bottomside sinusoidal irregularities in the equatorial F region. J. Geophys. Res. 88(A10), 8025-8042 (1983)

R.F. Woodman, Spread F - an old equatorial aeronomy problem finally resolved? Ann. Geophys. 27(5), 1915-1934 (2009). https://doi.org/10.5194/angeo-27-1915-2009

R.F. Woodman, E. Kudeki, A causal relationship between lightning and explosive spread F. Geophys. Res. Lett. 11(12), 1165-1167 (1984)

T.N. Woods, F.G. Eparvier, S.M. Bailey, P.C. Chamberlin, J. Lean, G.J. Rottman, S.C. Solomon, W.K. Tobiska, D.L. Woodraska, Solar EUV Experiment (SEE): mission overview and first results. J. Geophys. Res. 110, A01312 (2005)

Publisher's Note Springer Nature remains neutral with regard to jurisdictional claims in published maps and institutional affiliations.

\section{Authors and Affiliations}

R. Pfaff ${ }^{1}$ (D) P. Uribe ${ }^{1} \cdot$ R. Fourre ${ }^{1} \cdot$ J. Kujawski ${ }^{1} \cdot$ N. Maynard ${ }^{2} \cdot$ M. Acuña ${ }^{1} \cdot$ D. Rowland ${ }^{1}$. H. Freudenreich ${ }^{1} \cdot$ K. Bromund ${ }^{1} \cdot$ S. Martin ${ }^{1} \cdot$ C. Liebrecht ${ }^{1} \cdot$ R. Kramer ${ }^{3} \cdot$ F. Hunsaker ${ }^{1}$.

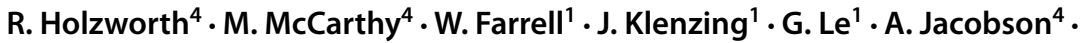
J. Houser ${ }^{1} \cdot$ C. Steigies ${ }^{1} \cdot$ J.-J. Berthelier ${ }^{5}$

R. Pfaff

Robert.F.Pfaff@nasa.gov

1 NASA Goddard Space Flight Center, Greenbelt, MD, USA

2 Mission Research Corporation, Nashua, NH, USA

3 Orbital Sciences Corporation, Beltsville, MD, USA

4 University of Washington, Seattle, WA, USA

5 CETP/IPSL, Saint-Maur, France 